\title{
Phosphine Coordination Complexes of the Diphenylphosphenium Cation: A Versatile Synthetic Methodology for P-P Bond Formation
}

\author{
Neil Burford ${ }^{* a}$, Paul J. Ragogna ${ }^{a}$, Robert McDonald ${ }^{b}$ and Michael J. Ferguson ${ }^{b}$ \\ Supporting Information Package for X-Ray \\ Crystallographic Data
}

${ }^{a}$ Department of Chemistry, Dalhousie University, Halifax, Nova Scotia,

Canada, B3H 4J6

${ }^{b}$ X-Ray Crystallography Laboratory, Department of Chemistry, University of Alberta, Edmonton, Alberta T6G 2G2

Ph: (902) 494-3681; Fax: (905) 494-1310; Neil.Burford@dal.ca 
Figure S1: Figure S1: View of $\mathbf{2 c O S O}_{2} \mathbf{C F}_{\mathbf{3}}$ with hydrogen atoms removed. This highlights the complete numbering scheme.

FigureS2: View of $\mathbf{2 a G a C l}_{\mathbf{4}}$ with hydrogen atoms removed. This highlights the complete numbering scheme.

Figure S3: Solid state structure of $\underline{\mathbf{3}}\left[\mathrm{GaCl}_{4}\right]$ including complete numbering scheme. Ellipsoides are $50 \%$ probability. Hydrogen atoms are removed for clarity and symmetry equivalent atoms are not shown.

Figure S4: Solid state structure of $\mathbf{4 O S O}_{2} \mathbf{C F}_{3}$ including complete numbering scheme. Ellipsoides are 50\% probability. Hydrogen atoms are removed for clarity and symmetry equivalent atoms are not shown.

Figure S5: Soilid State structure of $\mathbf{2 d O S O} \mathbf{C F}_{3}$ with full labeling scheme. Ellipsoids are 50\% probability and hydrogen atoms are removed for clarity.

Table S1: Crystal data and structure refinement for $\mathbf{2} \mathbf{c O S O}_{2} \mathbf{C F}_{\mathbf{3}}$.

Table S2: Atomic coordinates ( $\left.\times 10^{4}\right)$ and equivalent isotropic displacement parameters $\left(\AA^{2} \times 10^{3}\right)$ for $2 \mathbf{C O S O}_{2} \mathbf{C F}_{3}$. U(eq) is defined as one third of the trace of the orthogonalized $\mathrm{U}^{\mathrm{ij}}$ tensor.

Table S3: Bond lengths $[\AA]$ and angles $\left[^{\circ}\right]$ for $\mathbf{2} \mathbf{c O S O}_{2} \mathbf{C F}_{3}$.

Table S4: Anisotropic displacement parameters $\left(\AA^{2} \times 10^{3}\right)$ for $\mathbf{2 c} \mathbf{O S O}_{2} \mathbf{C F}_{3}$. The anisotropic displacement factor exponent takes the form: $-2 \pi^{2}\left[h^{2} a^{* 2} U^{11}+\ldots+2 h k\right.$ a* b* $\left.U^{12}\right]$

Table S5: Torsion angles $\left[{ }^{\circ}\right]$ for $\mathbf{2} \mathbf{c O S O}_{2} \mathbf{C F}_{3}$.

Table S6: Crystal data and structure refinement for $\mathbf{2} \mathbf{a G a C l} \mathbf{4}$.

Table S7: Atomic coordinates ( $\left.\times 10^{4}\right)$ and equivalent isotropic displacement parameters $\left(\AA^{2} \times 10^{3}\right)$ for $\mathbf{2 a G a C l}_{\mathbf{4}}$. U(eq) is defined as one third of the trace of the orthogonalized Uij tensor.

Table S8: Bond lengths $[\AA]$ for $\mathbf{2} \mathbf{a G a C l} \mathbf{H}_{\mathbf{4}}$

Table S9: Bond angles $\left[{ }^{\circ}\right]$ for $\mathbf{2} \mathbf{a G a C l} \mathbf{l}_{\mathbf{4}}$.

Table S10: Selected torsion angles $\left[^{\circ}\right]$ for $\mathbf{2} \mathbf{a G a C l}$.

Table S11: Selected contact distances $[\AA]$ and angles $\left[{ }^{\circ}\right]$ and best plane calculations for $\mathbf{2 a G a C l} \mathbf{G}_{\mathbf{4}}$.

Table S12: Anisotropic displacement parameters $\left(\AA^{2} \times 10^{3}\right)$ for $\mathbf{2} \mathbf{a G a C l} \mathbf{G}_{\mathbf{4}}$. The anisotropic displacement factor exponent takes the form:

$$
-2 \mathrm{pi}^{2}\left[\mathrm{~h}^{2} \mathrm{a}^{* 2} \mathrm{U} 11+\ldots+2 \mathrm{hk} \mathrm{a} \mathrm{b}^{*} \mathrm{U} 12\right]
$$

Table S13: Hydrogen coordinates $\left(\times 10^{4}\right)$ and isotropic displacement parameters $\left(\AA^{2} \times 10^{3}\right)$ for $\mathbf{2} \mathbf{a G a C l} \mathbf{G}_{\mathbf{4}}$.

Table S14: Crystal data and structure refinement for dal0303 1-diphosphino-2-phosphoniumbenze $\left(3 \mathrm{GaCl} 4 \bullet 0.5 \mathrm{CH}_{2} \mathrm{Cl}_{2}\right)$.

Table S15: Atomic coordinates ( $\left.\mathrm{x} 10^{4}\right)$ and equivalent isotropic displacement parameters $\left(\AA^{2} \times 10^{3}\right)$ 
for dal0303 1-diphosphino-2-phosphoniumbenze $\left(\mathbf{3} \mathbf{G a C l 4} \bullet \mathbf{0 . 5} \mathbf{C H}_{\mathbf{2}} \mathbf{C l} \mathbf{2}\right)$. U(eq) is defined as one third of the trace of the orthogonalized $\mathrm{Uij}$ tensor.

Table S16: Bond lengths $[\AA]$ and angles $\left[^{\circ}\right]$ for da10303 1-diphosphino-2-phosphoniumbenze $\left(3 \mathrm{GaCl} \bullet \bullet .5 \mathrm{CH}_{2} \mathrm{Cl}_{2}\right)$.

Table S17: Anisotropic displacement parameters $\left(\AA^{2} \times 10^{3}\right)$ for dal0303 1-diphosphino-2-phosphoniumbenze $\left(\mathbf{3 G a C l 4} \bullet \mathbf{0 . 5} \mathbf{C H}_{2} \mathbf{C l}_{2}\right)$. The anisotropic displacement factor exponent takes the form: $-2 \pi^{2}\left[\mathrm{~h}^{2} \mathrm{a}^{2} \mathrm{U}^{11}+\ldots+\right.$ $2 \mathrm{~h} \mathrm{k} \mathrm{a}^{*} \mathrm{~b}^{*} \mathrm{U}^{12}$ ]

Table S19: Torsion angles $\left[{ }^{\circ}\right]$ for da10303 1-diphosphino-2-phosphoniumbenze $\left(\mathbf{3 G a C l 4 \bullet} \mathbf{0 . 5} \mathbf{C H}_{\mathbf{2}} \mathbf{C l}_{\mathbf{2}}\right)$.

Table S20: Crystal data and structure refinement for $\mathbf{4 O S O}_{2} \mathbf{C F}_{3}$.

Table S21: Atomic coordinates ( x 104) and equivalent isotropic displacement parameters $\left(\AA^{2} \times 10^{3}\right)$ for $\mathbf{4 O S O}_{2} \mathbf{C F}_{3}$. U(eq) is defined as one third of the trace of the orthogonalized $\mathrm{Uij}^{\mathrm{ij}}$ tensor.

Table S22: Bond lengths $[\AA]$ and angles $\left[^{\circ}\right]$ for $\mathbf{4 O S O}_{2} \mathbf{C F}_{3}$.

Table S23: Anisotropic displacement parameters $\left(\AA^{2} \times 10^{3}\right)$ for $\mathbf{4 O S O}_{2} \mathbf{C F}_{\mathbf{3}}$. The anisotropic displacement factor exponent takes the form: $-2 \pi^{2}\left[\mathrm{~h}^{2} \mathrm{a}^{* 2} \mathrm{U}^{11}+\ldots+2 \mathrm{hk} \mathrm{a}^{*} \mathrm{~b}^{*} \mathrm{U}^{12}\right]$

Table S24: Torsion angles $\left[^{\circ}\right]$ for $\mathbf{4 O S O}_{2} \mathbf{C F}_{3}$.

Table S25: Crystal data and structure refinement for $\mathbf{2} \mathbf{d O S O}_{2} \mathbf{C F}_{3}$.

Table S26: Atomic coordinates ( $\left.\mathrm{x} 10^{4}\right)$ and equivalent isotropic displacement parameters $\left(\AA^{2} \times 10^{3}\right)$ for $\mathbf{2} \mathbf{d O S O}_{2} \mathbf{C F}_{3}$. U(eq) is defined as one third of the trace of the orthogonalized $\mathrm{U}^{\mathrm{ij}}$ tensor.

Table S27: Bond lengths $[\AA \AA]$ and angles $\left[^{\circ}\right]$ for $\mathbf{2 d O S O}_{\mathbf{2}} \mathbf{C F}_{\mathbf{3}}$

Table S28: Anisotropic displacement parameters $\left(\AA^{2} \times 10^{3}\right)$ for $\mathbf{2 d O S O} \mathbf{C F}_{\mathbf{3}}$. The anisotropic displacement factor exponent takes the form: $-2 \pi^{2}\left[\mathrm{~h}^{2} \mathrm{a}^{* 2} \mathrm{U}^{11}+\ldots+2 \mathrm{hk} \mathrm{a}^{*} \mathrm{~b}^{*} \mathrm{U}^{12}\right]$

Table S29. Torsion angles $\left[{ }^{\circ}\right]$ for $\mathbf{2} \mathbf{d O S O}_{2} \mathbf{C F}_{3}$. 


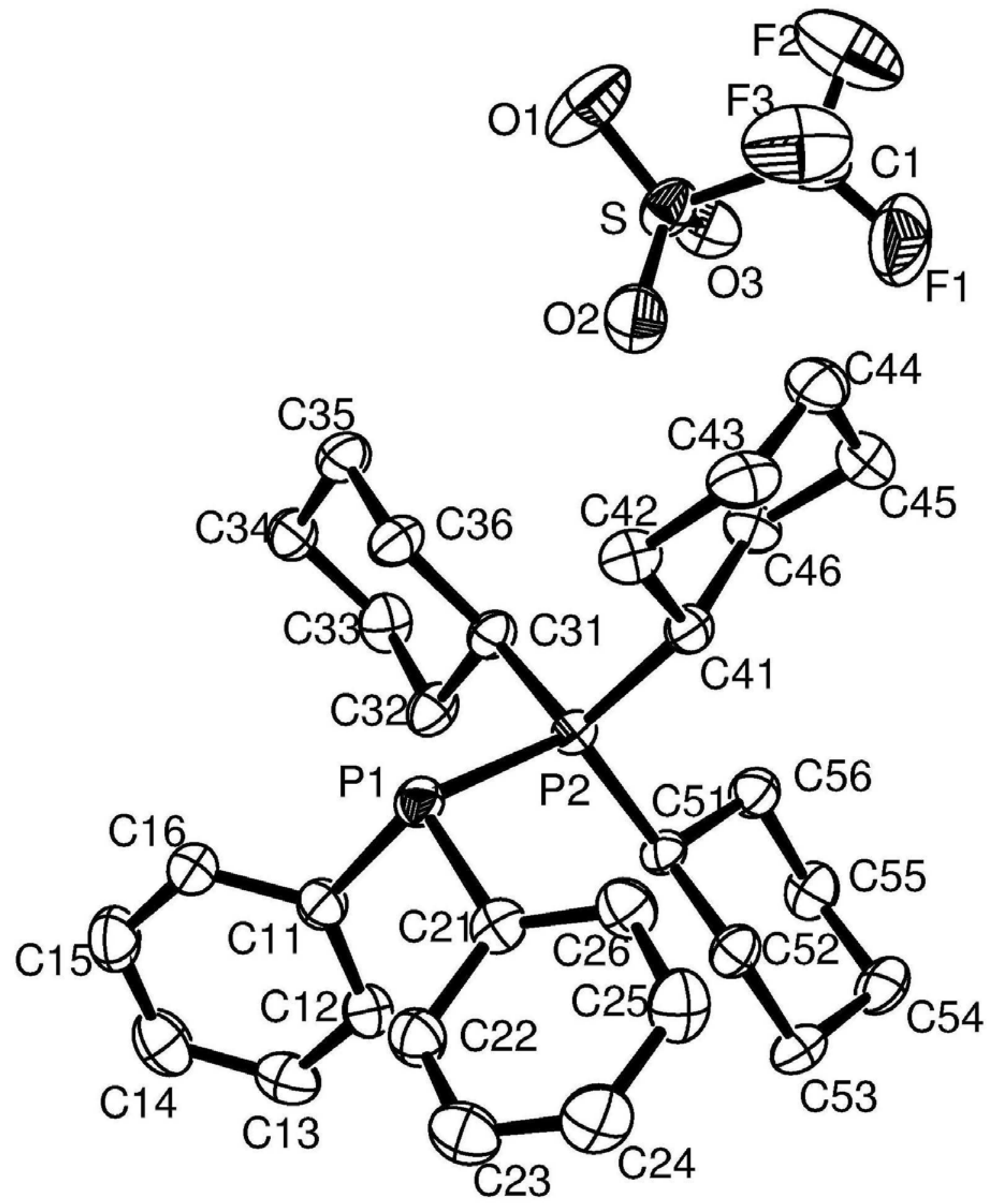

Figure S1: View of $\mathbf{2} \mathbf{C O S O}_{\mathbf{2}} \mathbf{C F}_{\mathbf{3}}$ with hydrogen atoms removed. This highlights the complete numbering scheme. 
Table S1. Crystal data and structure refinement for $\mathbf{2 C O S O}_{2} \mathbf{C F}_{3}$.

Identification code

Empirical formula

Formula weight

Temperature

Wavelength

Crystal system

Space group

Unit cell dimensions

Volume

Z

Density (calculated)

Absorption coefficient

$\mathrm{F}(000)$

Crystal size

Theta range for data collection

Index ranges

Reflections collected

Independent reflections

Completeness to theta $=26.39^{\circ}$

Absorption correction

Max. and min. transmission

Refinement method

Data / restraints / parameters

Goodness-of-fit on $\mathrm{F}^{2}$

Final $\mathrm{R}$ indices [I $>2 \operatorname{sigma}(\mathrm{I})]$

$\mathrm{R}$ indices (all data)

Absolute structure parameter

Largest diff. peak and hole dal0245

C31 H43 F3 O3 P2 S

614.65

193(2) K

$0.71073 \AA$

Orthorhombic

P n a 21

$\mathrm{a}=18.934(3) \AA \quad \alpha=90^{\circ}$.

$\mathrm{b}=10.4510(16) \AA \quad \beta=90^{\circ}$.

$\mathrm{c}=15.711(2) \AA \quad \gamma=90^{\circ}$.

$3108.8(8) \AA^{3}$

4

$1.313 \mathrm{Mg} / \mathrm{m}^{3}$

$0.256 \mathrm{~mm}^{-1}$

1304

$0.97 \times 0.22 \times 0.08 \mathrm{~mm}^{3}$

2.15 to $26.39^{\circ}$.

$-23<=\mathrm{h}<=22,-12<=\mathrm{k}<=13,-18<=\mathrm{k}<=19$

16461

$6083[\mathrm{R}(\mathrm{int})=0.0456]$

$99.8 \%$

Semi-empirical from equivalents

0.9798 and 0.7895

Full-matrix least-squares on $\mathrm{F}^{2}$

$6083 / 1 / 361$

1.020

$\mathrm{R} 1=0.0410, \mathrm{wR} 2=0.0798$

$\mathrm{R} 1=0.0560, \mathrm{wR} 2=0.0850$

$0.01(6)$

0.309 and -0.200 e. $\AA^{-3}$ 
Table S2. Atomic coordinates ( x 104) and equivalent isotropic displacement parameters $\left(\AA^{2} \times 10^{3}\right)$ for $\mathbf{2 C O S O}_{2} \mathbf{C F}_{3}$. U(eq) is defined as one third of the trace of the orthogonalized $U^{i j}$ tensor.

\begin{tabular}{|c|c|c|c|c|}
\hline & $\mathrm{x}$ & $\mathrm{y}$ & $\mathrm{z}$ & $\mathrm{U}(\mathrm{eq})$ \\
\hline- & & & & \\
\hline $\mathrm{P}(1)$ & $1274(1)$ & $1746(1)$ & $5063(1)$ & $27(1)$ \\
\hline $\mathrm{P}(2)$ & $1128(1)$ & $476(1)$ & $3944(1)$ & $22(1)$ \\
\hline$C(11)$ & $362(1)$ & $2125(2)$ & $5359(2)$ & $28(1)$ \\
\hline$C(12)$ & $-145(1)$ & $1234(3)$ & $5603(2)$ & $32(1)$ \\
\hline$C(13)$ & $-821(1)$ & $1632(3)$ & $5833(2)$ & $39(1)$ \\
\hline$C(14)$ & $-989(2)$ & 2921(3) & $5826(2)$ & $44(1)$ \\
\hline$C(15)$ & $-489(2)$ & $3816(3)$ & $5588(2)$ & $43(1)$ \\
\hline$C(16)$ & $180(1)$ & $3419(3)$ & $5356(2)$ & $33(1)$ \\
\hline $\mathrm{C}(21)$ & $1568(1)$ & $718(3)$ & $5945(2)$ & $27(1)$ \\
\hline$C(22)$ & $1338(1)$ & $1055(3)$ & $6758(2)$ & $33(1)$ \\
\hline$C(23)$ & $1613(2)$ & $433(3)$ & $7464(2)$ & $40(1)$ \\
\hline $\mathrm{C}(24)$ & $2098(2)$ & $-528(3)$ & $7371(2)$ & $41(1)$ \\
\hline$C(25)$ & $2329(2)$ & $-879(3)$ & $6579(2)$ & $39(1)$ \\
\hline$C(26)$ & $2074(1)$ & $-238(3)$ & $5860(2)$ & $34(1)$ \\
\hline$C(31)$ & $734(1)$ & $1483(2)$ & $3114(2)$ & $26(1)$ \\
\hline$C(32)$ & $-73(1)$ & $1635(3)$ & $3182(2)$ & $32(1)$ \\
\hline C(33) & $-351(1)$ & $2357(3)$ & $2408(2)$ & $36(1)$ \\
\hline$C(34)$ & $1(1)$ & $3660(3)$ & $2332(2)$ & $33(1)$ \\
\hline$C(35)$ & $798(1)$ & $3549(3)$ & $2275(2)$ & $37(1)$ \\
\hline$C(36)$ & $1095(1)$ & 2794(3) & $3033(2)$ & $32(1)$ \\
\hline $\mathrm{C}(41)$ & $2017(1)$ & $-40(2)$ & $3598(2)$ & $25(1)$ \\
\hline$C(42)$ & $2620(1)$ & $900(3)$ & $3818(2)$ & $32(1)$ \\
\hline$C(43)$ & $3334(1)$ & $308(3)$ & $3586(2)$ & $38(1)$ \\
\hline$C(44)$ & $3360(1)$ & $-96(3)$ & $2658(2)$ & $41(1)$ \\
\hline$C(45)$ & $2755(1)$ & $-977(3)$ & $2439(2)$ & $39(1)$ \\
\hline$C(46)$ & $2049(1)$ & $-338(3)$ & $2643(2)$ & $30(1)$ \\
\hline $\mathrm{C}(51)$ & $558(1)$ & $-937(2)$ & $4071(2)$ & $24(1)$ \\
\hline$C(52)$ & $798(1)$ & $-1837(2)$ & $4785(2)$ & $28(1)$ \\
\hline$C(53)$ & $279(1)$ & $-2951(2)$ & $4890(2)$ & $33(1)$ \\
\hline$C(54)$ & $174(2)$ & $-3673(3)$ & $4058(2)$ & $37(1)$ \\
\hline
\end{tabular}




$\begin{array}{lrrrr}\mathrm{C}(55) & -57(1) & -2784(3) & 3356(2) & 35(1) \\ \mathrm{C}(56) & 473(1) & -1699(3) & 3239(2) & 31(1) \\ \mathrm{S} & 1790(1) & 1421(1) & 44(1) & 39(1) \\ \mathrm{F}(1) & 2394(1) & -824(2) & 111(2) & 89(1) \\ \mathrm{F}(2) & 2826(1) & 420(3) & -834(2) & 96(1) \\ \mathrm{F}(3) & 3073(1) & 712(3) & 468(2) & 82(1) \\ \mathrm{O}(1) & 2054(2) & 2630(2) & -233(2) & 79(1) \\ \mathrm{O}(2) & 1628(1) & 1339(2) & 930(1) & 53(1) \\ \mathrm{O}(3) & 1279(1) & 837(2) & -500(1) & 48(1) \\ \mathrm{C}(1) & 2555(2) & 378(4) & -51(2) & 57(1)\end{array}$


Table S3. Bond lengths $[\AA]$ and angles $\left[{ }^{\circ}\right]$ for $\mathbf{2} \mathbf{C O S O}_{2} \mathbf{C F}_{3}$.

\begin{tabular}{ll}
\hline $\mathrm{P}(1)-\mathrm{C}(11)$ & $1.831(3)$ \\
$\mathrm{P}(1)-\mathrm{C}(21)$ & $1.838(3)$ \\
$\mathrm{P}(1)-\mathrm{P}(2)$ & $2.2205(10)$ \\
$\mathrm{P}(2)-\mathrm{C}(31)$ & $1.834(2)$ \\
$\mathrm{P}(2)-\mathrm{C}(51)$ & $1.839(2)$ \\
$\mathrm{P}(2)-\mathrm{C}(41)$ & $1.848(2)$ \\
$\mathrm{C}(11)-\mathrm{C}(12)$ & $1.392(4)$ \\
$\mathrm{C}(11)-\mathrm{C}(16)$ & $1.395(4)$ \\
$\mathrm{C}(12)-\mathrm{C}(13)$ & $1.393(4)$ \\
$\mathrm{C}(13)-\mathrm{C}(14)$ & $1.385(4)$ \\
$\mathrm{C}(14)-\mathrm{C}(15)$ & $1.383(4)$ \\
$\mathrm{C}(15)-\mathrm{C}(16)$ & $1.382(4)$ \\
$\mathrm{C}(21)-\mathrm{C}(26)$ & $1.391(4)$ \\
$\mathrm{C}(21)-\mathrm{C}(22)$ & $1.395(4)$ \\
$\mathrm{C}(22)-\mathrm{C}(23)$ & $1.387(4)$ \\
$\mathrm{C}(23)-\mathrm{C}(24)$ & $1.369(4)$ \\
$\mathrm{C}(24)-\mathrm{C}(25)$ & $1.370(4)$ \\
$\mathrm{C}(25)-\mathrm{C}(26)$ & $1.398(4)$ \\
$\mathrm{C}(31)-\mathrm{C}(36)$ & $1.537(3)$ \\
$\mathrm{C}(31)-\mathrm{C}(32)$ & $1.540(3)$ \\
$\mathrm{C}(32)-\mathrm{C}(33)$ & $1.524(4)$ \\
$\mathrm{C}(33)-\mathrm{C}(34)$ & $1.521(4)$ \\
$\mathrm{C}(34)-\mathrm{C}(35)$ & $1.515(4)$ \\
$\mathrm{C}(35)-\mathrm{C}(36)$ & $1.535(4)$ \\
$\mathrm{C}(41)-\mathrm{C}(46)$ & $1.534(3)$ \\
$\mathrm{C}(41)-\mathrm{C}(42)$ & $1.546(3)$ \\
$\mathrm{C}(42)-\mathrm{C}(43)$ & $1.531(4)$ \\
$\mathrm{C}(43)-\mathrm{C}(44)$ & $1.519(4)$ \\
$\mathrm{C}(44)-\mathrm{C}(45)$ & $1.509(4)$ \\
$\mathrm{C}(45)-\mathrm{C}(46)$ & $1.528(4)$ \\
$\mathrm{C}(51)-\mathrm{C}(52)$ & $1.533(3)$ \\
$\mathrm{C}(51)-\mathrm{C}(56)$ & $1.539(4)$ \\
$\mathrm{C}(52)-\mathrm{C}(53)$ & $1.533(3)$ \\
$\mathrm{C}(53)-\mathrm{C}(54)$ & $1.522(4)$ \\
$\mathrm{C}(54)-\mathrm{C}(55)$ & \\
& \\
&
\end{tabular}




\begin{tabular}{|c|c|}
\hline$C(55)-C(56)$ & $1.527(4)$ \\
\hline $\mathrm{S}-\mathrm{O}(1)$ & $1.427(2)$ \\
\hline $\mathrm{S}-\mathrm{O}(3)$ & $1.428(2)$ \\
\hline $\mathrm{S}-\mathrm{O}(2)$ & $1.429(2)$ \\
\hline $\mathrm{S}-\mathrm{C}(1)$ & $1.820(4)$ \\
\hline $\mathrm{F}(1)-\mathrm{C}(1)$ & $1.317(4)$ \\
\hline $\mathrm{F}(2)-\mathrm{C}(1)$ & $1.333(4)$ \\
\hline $\mathrm{F}(3)-\mathrm{C}(1)$ & $1.321(4)$ \\
\hline $\mathrm{C}(11)-\mathrm{P}(1)-\mathrm{C}(21)$ & $102.78(12)$ \\
\hline $\mathrm{C}(11)-\mathrm{P}(1)-\mathrm{P}(2)$ & $102.28(9)$ \\
\hline $\mathrm{C}(21)-\mathrm{P}(1)-\mathrm{P}(2)$ & $106.55(9)$ \\
\hline $\mathrm{C}(31)-\mathrm{P}(2)-\mathrm{C}(51)$ & $107.40(11)$ \\
\hline $\mathrm{C}(31)-\mathrm{P}(2)-\mathrm{C}(41)$ & $109.19(12)$ \\
\hline $\mathrm{C}(51)-\mathrm{P}(2)-\mathrm{C}(41)$ & $109.37(12)$ \\
\hline $\mathrm{C}(31)-\mathrm{P}(2)-\mathrm{P}(1)$ & $105.72(9)$ \\
\hline $\mathrm{C}(51)-\mathrm{P}(2)-\mathrm{P}(1)$ & $117.82(8)$ \\
\hline $\mathrm{C}(41)-\mathrm{P}(2)-\mathrm{P}(1)$ & $107.08(8)$ \\
\hline$C(12)-C(11)-C(16)$ & $118.6(2)$ \\
\hline $\mathrm{C}(12)-\mathrm{C}(11)-\mathrm{P}(1)$ & $125.1(2)$ \\
\hline$C(16)-C(11)-P(1)$ & $116.2(2)$ \\
\hline $\mathrm{C}(11)-\mathrm{C}(12)-\mathrm{C}(13)$ & $120.4(3)$ \\
\hline$C(14)-C(13)-C(12)$ & $120.0(3)$ \\
\hline$C(15)-C(14)-C(13)$ & $120.2(3)$ \\
\hline$C(16)-C(15)-C(14)$ & $119.7(3)$ \\
\hline$C(15)-C(16)-C(11)$ & $121.1(3)$ \\
\hline$C(26)-C(21)-C(22)$ & $118.9(2)$ \\
\hline $\mathrm{C}(26)-\mathrm{C}(21)-\mathrm{P}(1)$ & $123.8(2)$ \\
\hline $\mathrm{C}(22)-\mathrm{C}(21)-\mathrm{P}(1)$ & $116.6(2)$ \\
\hline $\mathrm{C}(23)-\mathrm{C}(22)-\mathrm{C}(21)$ & $119.8(3)$ \\
\hline $\mathrm{C}(24)-\mathrm{C}(23)-\mathrm{C}(22)$ & $120.7(3)$ \\
\hline $\mathrm{C}(23)-\mathrm{C}(24)-\mathrm{C}(25)$ & $120.5(3)$ \\
\hline$C(24)-C(25)-C(26)$ & $119.7(3)$ \\
\hline $\mathrm{C}(21)-\mathrm{C}(26)-\mathrm{C}(25)$ & $120.3(3)$ \\
\hline $\mathrm{C}(36)-\mathrm{C}(31)-\mathrm{C}(32)$ & $110.8(2)$ \\
\hline $\mathrm{C}(36)-\mathrm{C}(31)-\mathrm{P}(2)$ & $112.91(17)$ \\
\hline $\mathrm{C}(32)-\mathrm{C}(31)-\mathrm{P}(2)$ & $114.44(17)$ \\
\hline
\end{tabular}




$\begin{array}{ll}\mathrm{C}(33)-\mathrm{C}(32)-\mathrm{C}(31) & 109.8(2) \\ \mathrm{C}(34)-\mathrm{C}(33)-\mathrm{C}(32) & 110.8(2) \\ \mathrm{C}(35)-\mathrm{C}(34)-\mathrm{C}(33) & 111.8(2) \\ \mathrm{C}(34)-\mathrm{C}(35)-\mathrm{C}(36) & 111.0(2) \\ \mathrm{C}(35)-\mathrm{C}(36)-\mathrm{C}(31) & 111.1(2) \\ \mathrm{C}(46)-\mathrm{C}(41)-\mathrm{C}(42) & 108.6(2) \\ \mathrm{C}(46)-\mathrm{C}(41)-\mathrm{P}(2) & 112.52(17) \\ \mathrm{C}(42)-\mathrm{C}(41)-\mathrm{P}(2) & 114.97(18) \\ \mathrm{C}(43)-\mathrm{C}(42)-\mathrm{C}(41) & 110.1(2) \\ \mathrm{C}(44)-\mathrm{C}(43)-\mathrm{C}(42) & 111.7(2) \\ \mathrm{C}(45)-\mathrm{C}(44)-\mathrm{C}(43) & 111.4(2) \\ \mathrm{C}(44)-\mathrm{C}(45)-\mathrm{C}(46) & 110.5(2) \\ \mathrm{C}(45)-\mathrm{C}(46)-\mathrm{C}(41) & 109.2(2) \\ \mathrm{C}(52)-\mathrm{C}(51)-\mathrm{C}(56) & 109.6(2) \\ \mathrm{C}(52)-\mathrm{C}(51)-\mathrm{P}(2) & 113.47(16) \\ \mathrm{C}(56)-\mathrm{C}(51)-\mathrm{P}(2) & 112.59(17) \\ \mathrm{C}(53)-\mathrm{C}(52)-\mathrm{C}(51) & 110.8(2) \\ \mathrm{C}(54)-\mathrm{C}(53)-\mathrm{C}(52) & 111.6(2) \\ \mathrm{C}(55)-\mathrm{C}(54)-\mathrm{C}(53) & 111.2(2) \\ \mathrm{C}(54)-\mathrm{C}(55)-\mathrm{C}(56) & 110.8(2) \\ \mathrm{C}(55)-\mathrm{C}(56)-\mathrm{C}(51) & 110.6(2) \\ \mathrm{O}(1)-\mathrm{S}-\mathrm{O}(3) & 115.69(15) \\ \mathrm{O}(1)-\mathrm{S}-\mathrm{O}(2) & 115.18(15) \\ \mathrm{O}(3)-\mathrm{S}-\mathrm{O}(2) & 114.34(14) \\ \mathrm{O}(1)-\mathrm{S}-\mathrm{C}(1) & 103.11(18) \\ \mathrm{O}(3)-\mathrm{S}-\mathrm{C}(1) & 103.57(15) \\ \mathrm{O}(2)-\mathrm{S}-\mathrm{C}(1) & 102.42(15) \\ \mathrm{F}(1)-\mathrm{C}(1)-\mathrm{F}(3) & 107.8(3) \\ \mathrm{F}(1)-\mathrm{C}(1)-\mathrm{F}(2) & 107.4(3) \\ \mathrm{F}(3)-\mathrm{C}(1)-\mathrm{F}(2) & 106.0(3) \\ \mathrm{F}(1)-\mathrm{C}(1)-\mathrm{S} & 111.8(2) \\ \mathrm{F}(3)-\mathrm{C}(1)-\mathrm{S} & 112.4(3) \\ \mathrm{F}(2)-\mathrm{C}(1)-\mathrm{S} & 111.2(3) \\ & \\ & \end{array}$

Symmetry transformations used to generate equivalent atoms: 
Table S4. Anisotropic displacement parameters $\left(\AA^{2} x 10^{3}\right)$ for $\mathbf{2} \mathbf{c O S O} \mathbf{O S F}_{\mathbf{3}}$. The anisotropic displacement factor exponent takes the form: $-2 \pi^{2}\left[h^{2} a^{* 2} U^{11}+\ldots+2 h k a^{*} b^{*} U^{12}\right]$

\begin{tabular}{|c|c|c|c|c|c|c|}
\hline & $\mathrm{U}^{11}$ & $\mathrm{U}^{22}$ & $\mathrm{U}^{33}$ & $\mathrm{U}^{23}$ & $\mathrm{U}^{13}$ & $\mathrm{U}^{12}$ \\
\hline $\mathrm{P}(1)$ & $30(1)$ & $23(1)$ & $27(1)$ & $-1(1)$ & $0(1)$ & $-3(1)$ \\
\hline $\mathrm{P}(2)$ & $23(1)$ & $21(1)$ & $23(1)$ & $2(1)$ & $-1(1)$ & $-1(1)$ \\
\hline $\mathrm{C}(11)$ & $32(1)$ & $28(2)$ & $25(1)$ & $-1(1)$ & $-1(1)$ & $0(1)$ \\
\hline$C(12)$ & $38(2)$ & $28(2)$ & $30(2)$ & $-4(1)$ & $2(1)$ & $-1(1)$ \\
\hline$C(13)$ & $34(2)$ & $49(2)$ & $32(2)$ & $-3(1)$ & $4(1)$ & $-8(1)$ \\
\hline$C(14)$ & $35(2)$ & $54(2)$ & $44(2)$ & $-7(2)$ & $0(1)$ & $11(2)$ \\
\hline$C(15)$ & $50(2)$ & $34(2)$ & $44(2)$ & $-2(1)$ & $0(2)$ & $12(1)$ \\
\hline$C(16)$ & $40(2)$ & $27(2)$ & $31(2)$ & 1(1) & $4(1)$ & $0(1)$ \\
\hline$C(21)$ & $26(1)$ & $28(2)$ & $27(1)$ & $-1(1)$ & $-6(1)$ & $-5(1)$ \\
\hline$C(22)$ & $36(2)$ & $33(2)$ & $29(2)$ & $-2(1)$ & $-1(1)$ & $1(1)$ \\
\hline $\mathrm{C}(23)$ & $44(2)$ & $50(2)$ & $26(2)$ & $-4(1)$ & $-3(1)$ & $-2(2)$ \\
\hline $\mathrm{C}(24)$ & $50(2)$ & $47(2)$ & $27(2)$ & $3(1)$ & $-13(1)$ & $2(2)$ \\
\hline$C(25)$ & $40(2)$ & $38(2)$ & $38(2)$ & $-2(1)$ & $-12(1)$ & $7(1)$ \\
\hline$C(26)$ & $34(1)$ & $38(2)$ & $28(2)$ & $-3(1)$ & $-3(1)$ & $1(1)$ \\
\hline $\mathrm{C}(31)$ & $29(1)$ & $25(1)$ & $24(1)$ & $5(1)$ & $-4(1)$ & $2(1)$ \\
\hline $\mathrm{C}(32)$ & $27(1)$ & $28(2)$ & $41(2)$ & $5(1)$ & $-4(1)$ & $-1(1)$ \\
\hline$C(33)$ & $36(2)$ & $37(2)$ & $34(2)$ & $0(1)$ & $-8(1)$ & $6(1)$ \\
\hline$C(34)$ & $36(2)$ & $31(2)$ & $31(2)$ & $7(1)$ & $-1(1)$ & $10(1)$ \\
\hline$C(35)$ & $40(2)$ & $33(2)$ & $37(2)$ & $11(1)$ & $5(1)$ & $6(1)$ \\
\hline$C(36)$ & $29(1)$ & $29(2)$ & $38(2)$ & $9(1)$ & $0(1)$ & $-1(1)$ \\
\hline$C(41)$ & $21(1)$ & $25(1)$ & $30(1)$ & $3(1)$ & $0(1)$ & 1(1) \\
\hline$C(42)$ & $28(1)$ & $36(2)$ & $32(2)$ & $4(1)$ & $-3(1)$ & $-3(1)$ \\
\hline$C(43)$ & $23(1)$ & $49(2)$ & $43(2)$ & $12(1)$ & $-3(1)$ & $-4(1)$ \\
\hline$C(44)$ & $28(2)$ & $44(2)$ & $51(2)$ & $7(2)$ & $11(1)$ & $3(1)$ \\
\hline$C(45)$ & $37(2)$ & $41(2)$ & $39(2)$ & $-2(1)$ & $9(1)$ & $3(1)$ \\
\hline$C(46)$ & $27(1)$ & $38(2)$ & $26(2)$ & $0(1)$ & $0(1)$ & $-1(1)$ \\
\hline $\mathrm{C}(51)$ & $22(1)$ & $20(1)$ & $29(1)$ & $1(1)$ & $2(1)$ & $-5(1)$ \\
\hline$C(52)$ & $31(1)$ & $24(1)$ & $30(2)$ & $1(1)$ & $-4(1)$ & $-3(1)$ \\
\hline $\mathrm{C}(53)$ & $34(1)$ & $28(1)$ & $36(2)$ & $8(1)$ & $1(1)$ & $-5(1)$ \\
\hline$C(54)$ & $36(1)$ & $25(2)$ & $49(2)$ & $2(1)$ & $-2(1)$ & $-10(1)$ \\
\hline$C(55)$ & $36(2)$ & $31(2)$ & $37(2)$ & $-4(1)$ & $-7(1)$ & $-6(1)$ \\
\hline$C(56)$ & $36(1)$ & $28(2)$ & $29(2)$ & $0(1)$ & $0(1)$ & $-2(1)$ \\
\hline
\end{tabular}




$\begin{array}{lcccccc}\mathrm{S} & 56(1) & 33(1) & 30(1) & 4(1) & -7(1) & -2(1) \\ \mathrm{F}(1) & 59(1) & 52(1) & 156(2) & 2(2) & 13(2) & 16(1) \\ \mathrm{F}(2) & 51(1) & 169(3) & 69(2) & -16(2) & 13(1) & -2(1) \\ \mathrm{F}(3) & 47(1) & 125(2) & 74(2) & 24(1) & -20(1) & -18(1) \\ \mathrm{O}(1) & 125(2) & 47(2) & 65(2) & 22(1) & -24(2) & -27(2) \\ \mathrm{O}(2) & 80(2) & 48(1) & 31(1) & 1(1) & -3(1) & 10(1) \\ \mathrm{O}(3) & 45(1) & 63(2) & 36(1) & -2(1) & -4(1) & -2(1) \\ \mathrm{C}(1) & 40(2) & 80(3) & 50(2) & 1(2) & 2(2) & -10(2)\end{array}$


Table S5. Torsion angles $\left[{ }^{\circ}\right]$ for $\mathbf{2} \mathbf{c O S O}_{2} \mathbf{C F}_{3}$.

\begin{tabular}{lc}
\hline $\mathrm{C}(11)-\mathrm{P}(1)-\mathrm{P}(2)-\mathrm{C}(31)$ & $65.82(12)$ \\
$\mathrm{C}(21)-\mathrm{P}(1)-\mathrm{P}(2)-\mathrm{C}(31)$ & $173.33(11)$ \\
$\mathrm{C}(11)-\mathrm{P}(1)-\mathrm{P}(2)-\mathrm{C}(51)$ & $-54.17(12)$ \\
$\mathrm{C}(21)-\mathrm{P}(1)-\mathrm{P}(2)-\mathrm{C}(51)$ & $53.34(12)$ \\
$\mathrm{C}(11)-\mathrm{P}(1)-\mathrm{P}(2)-\mathrm{C}(41)$ & $-177.84(13)$ \\
$\mathrm{C}(21)-\mathrm{P}(1)-\mathrm{P}(2)-\mathrm{C}(41)$ & $-70.32(12)$ \\
$\mathrm{C}(21)-\mathrm{P}(1)-\mathrm{C}(11)-\mathrm{C}(12)$ & $-49.5(3)$ \\
$\mathrm{P}(2)-\mathrm{P}(1)-\mathrm{C}(11)-\mathrm{C}(12)$ & $60.9(2)$ \\
$\mathrm{C}(21)-\mathrm{P}(1)-\mathrm{C}(11)-\mathrm{C}(16)$ & $128.6(2)$ \\
$\mathrm{P}(2)-\mathrm{P}(1)-\mathrm{C}(11)-\mathrm{C}(16)$ & $-121.05(19)$ \\
$\mathrm{C}(16)-\mathrm{C}(11)-\mathrm{C}(12)-\mathrm{C}(13)$ & $0.5(4)$ \\
$\mathrm{P}(1)-\mathrm{C}(11)-\mathrm{C}(12)-\mathrm{C}(13)$ & $178.6(2)$ \\
$\mathrm{C}(11)-\mathrm{C}(12)-\mathrm{C}(13)-\mathrm{C}(14)$ & $-0.6(4)$ \\
$\mathrm{C}(12)-\mathrm{C}(13)-\mathrm{C}(14)-\mathrm{C}(15)$ & $0.3(4)$ \\
$\mathrm{C}(13)-\mathrm{C}(14)-\mathrm{C}(15)-\mathrm{C}(16)$ & $0.1(5)$ \\
$\mathrm{C}(14)-\mathrm{C}(15)-\mathrm{C}(16)-\mathrm{C}(11)$ & $-0.1(4)$ \\
$\mathrm{C}(12)-\mathrm{C}(11)-\mathrm{C}(16)-\mathrm{C}(15)$ & $-0.2(4)$ \\
$\mathrm{P}(1)-\mathrm{C}(11)-\mathrm{C}(16)-\mathrm{C}(15)$ & $-178.4(2)$ \\
$\mathrm{C}(11)-\mathrm{P}(1)-\mathrm{C}(21)-\mathrm{C}(26)$ & $150.6(2)$ \\
$\mathrm{P}(2)-\mathrm{P}(1)-\mathrm{C}(21)-\mathrm{C}(26)$ & $43.5(2)$ \\
$\mathrm{C}(11)-\mathrm{P}(1)-\mathrm{C}(21)-\mathrm{C}(22)$ & $-38.8(2)$ \\
$\mathrm{P}(2)-\mathrm{P}(1)-\mathrm{C}(21)-\mathrm{C}(22)$ & $-145.93(18)$ \\
$\mathrm{C}(26)-\mathrm{C}(21)-\mathrm{C}(22)-\mathrm{C}(23)$ & $-0.2(4)$ \\
$\mathrm{P}(1)-\mathrm{C}(21)-\mathrm{C}(22)-\mathrm{C}(23)$ & $-171.3(2)$ \\
$\mathrm{C}(21)-\mathrm{C}(22)-\mathrm{C}(23)-\mathrm{C}(24)$ & $-1.4(4)$ \\
$\mathrm{C}(22)-\mathrm{C}(23)-\mathrm{C}(24)-\mathrm{C}(25)$ & $1.1(5)$ \\
$\mathrm{C}(23)-\mathrm{C}(24)-\mathrm{C}(25)-\mathrm{C}(26)$ & $0.8(5)$ \\
$\mathrm{C}(22)-\mathrm{C}(21)-\mathrm{C}(26)-\mathrm{C}(25)$ & $2.2(4)$ \\
$\mathrm{P}(1)-\mathrm{C}(21)-\mathrm{C}(26)-\mathrm{C}(25)$ & $172.6(2)$ \\
$\mathrm{C}(24)-\mathrm{C}(25)-\mathrm{C}(26)-\mathrm{C}(21)$ & $-2.5(4)$ \\
$\mathrm{C}(51)-\mathrm{P}(2)-\mathrm{C}(31)-\mathrm{C}(36)$ & $171.47(18)$ \\
$\mathrm{C}(41)-\mathrm{P}(2)-\mathrm{C}(31)-\mathrm{C}(36)$ & $-70.0(2)$ \\
$\mathrm{P}(1)-\mathrm{P}(2)-\mathrm{C}(31)-\mathrm{C}(36)$ & $44.9(2)$ \\
$\mathrm{C}(51)-\mathrm{P}(2)-\mathrm{C}(31)-\mathrm{C}(32)$ & $43.5(2)$ \\
$\mathrm{C}(41)-\mathrm{P}(2)-\mathrm{C}(31)-\mathrm{C}(32)$ & \\
&
\end{tabular}




\begin{tabular}{|c|c|}
\hline $\mathrm{P}(1)-\mathrm{P}(2)-\mathrm{C}(31)-\mathrm{C}(32)$ & $-83.08(19)$ \\
\hline$C(36)-C(31)-C(32)-C(33)$ & $57.4(3)$ \\
\hline $\mathrm{P}(2)-\mathrm{C}(31)-\mathrm{C}(32)-\mathrm{C}(33)$ & $-173.55(19)$ \\
\hline $\mathrm{C}(31)-\mathrm{C}(32)-\mathrm{C}(33)-\mathrm{C}(34)$ & $-57.9(3)$ \\
\hline $\mathrm{C}(32)-\mathrm{C}(33)-\mathrm{C}(34)-\mathrm{C}(35)$ & $57.4(3)$ \\
\hline C(33)-C(34)-C(35)-C(36) & $-55.2(3)$ \\
\hline $\mathrm{C}(34)-\mathrm{C}(35)-\mathrm{C}(36)-\mathrm{C}(31)$ & $54.3(3)$ \\
\hline $\mathrm{C}(32)-\mathrm{C}(31)-\mathrm{C}(36)-\mathrm{C}(35)$ & $-55.8(3)$ \\
\hline $\mathrm{P}(2)-\mathrm{C}(31)-\mathrm{C}(36)-\mathrm{C}(35)$ & $174.37(19)$ \\
\hline $\mathrm{C}(31)-\mathrm{P}(2)-\mathrm{C}(41)-\mathrm{C}(46)$ & $-37.9(2)$ \\
\hline $\mathrm{C}(51)-\mathrm{P}(2)-\mathrm{C}(41)-\mathrm{C}(46)$ & $79.4(2)$ \\
\hline $\mathrm{P}(1)-\mathrm{P}(2)-\mathrm{C}(41)-\mathrm{C}(46)$ & $-151.90(17)$ \\
\hline $\mathrm{C}(31)-\mathrm{P}(2)-\mathrm{C}(41)-\mathrm{C}(42)$ & $87.1(2)$ \\
\hline $\mathrm{C}(51)-\mathrm{P}(2)-\mathrm{C}(41)-\mathrm{C}(42)$ & $-155.65(18)$ \\
\hline $\mathrm{P}(1)-\mathrm{P}(2)-\mathrm{C}(41)-\mathrm{C}(42)$ & $-26.9(2)$ \\
\hline$C(46)-C(41)-C(42)-C(43)$ & $-58.9(3)$ \\
\hline $\mathrm{P}(2)-\mathrm{C}(41)-\mathrm{C}(42)-\mathrm{C}(43)$ & $174.06(18)$ \\
\hline $\mathrm{C}(41)-\mathrm{C}(42)-\mathrm{C}(43)-\mathrm{C}(44)$ & $55.5(3)$ \\
\hline$C(42)-C(43)-C(44)-C(45)$ & $-54.4(3)$ \\
\hline $\mathrm{C}(43)-\mathrm{C}(44)-\mathrm{C}(45)-\mathrm{C}(46)$ & $56.7(3)$ \\
\hline$C(44)-C(45)-C(46)-C(41)$ & $-60.8(3)$ \\
\hline $\mathrm{C}(42)-\mathrm{C}(41)-\mathrm{C}(46)-\mathrm{C}(45)$ & $61.5(3)$ \\
\hline $\mathrm{P}(2)-\mathrm{C}(41)-\mathrm{C}(46)-\mathrm{C}(45)$ & $-170.09(19)$ \\
\hline $\mathrm{C}(31)-\mathrm{P}(2)-\mathrm{C}(51)-\mathrm{C}(52)$ & $-176.62(18)$ \\
\hline $\mathrm{C}(41)-\mathrm{P}(2)-\mathrm{C}(51)-\mathrm{C}(52)$ & $65.0(2)$ \\
\hline $\mathrm{P}(1)-\mathrm{P}(2)-\mathrm{C}(51)-\mathrm{C}(52)$ & $-57.5(2)$ \\
\hline $\mathrm{C}(31)-\mathrm{P}(2)-\mathrm{C}(51)-\mathrm{C}(56)$ & $58.2(2)$ \\
\hline $\mathrm{C}(41)-\mathrm{P}(2)-\mathrm{C}(51)-\mathrm{C}(56)$ & $-60.2(2)$ \\
\hline$P(1)-P(2)-C(51)-C(56)$ & $177.28(15)$ \\
\hline $\mathrm{C}(56)-\mathrm{C}(51)-\mathrm{C}(52)-\mathrm{C}(53)$ & $-56.4(3)$ \\
\hline $\mathrm{P}(2)-\mathrm{C}(51)-\mathrm{C}(52)-\mathrm{C}(53)$ & $176.79(17)$ \\
\hline $\mathrm{C}(51)-\mathrm{C}(52)-\mathrm{C}(53)-\mathrm{C}(54)$ & $55.4(3)$ \\
\hline $\mathrm{C}(52)-\mathrm{C}(53)-\mathrm{C}(54)-\mathrm{C}(55)$ & $-55.4(3)$ \\
\hline $\mathrm{C}(53)-\mathrm{C}(54)-\mathrm{C}(55)-\mathrm{C}(56)$ & $56.7(3)$ \\
\hline $\mathrm{C}(54)-\mathrm{C}(55)-\mathrm{C}(56)-\mathrm{C}(51)$ & $-58.5(3)$ \\
\hline $\mathrm{C}(52)-\mathrm{C}(51)-\mathrm{C}(56)-\mathrm{C}(55)$ & $58.1(3)$ \\
\hline $\mathrm{P}(2)-\mathrm{C}(51)-\mathrm{C}(56)-\mathrm{C}(55)$ & $-174.64(18)$ \\
\hline
\end{tabular}




$\begin{array}{lc}\text { O(1)-S-C(1)-F(1) } & -174.5(3) \\ \text { O(3)-S-C(1)-F(1) } & -53.6(3) \\ \text { O(2)-S-C(1)-F(1) } & 65.6(3) \\ \text { O(1)-S-C(1)-F(3) } & 64.2(3) \\ \text { O(3)-S-C(1)-F(3) } & -174.9(2) \\ \text { O(2)-S-C(1)-F(3) } & -55.7(3) \\ \text { O(1)-S-C(1)-F(2) } & -54.5(3) \\ \text { O(3)-S-C(1)-F(2) } & 66.4(3) \\ \text { O(2)-S-C(1)-F(2) } & -174.4(2)\end{array}$

Symmetry transformations used to generate equivalent atoms: 


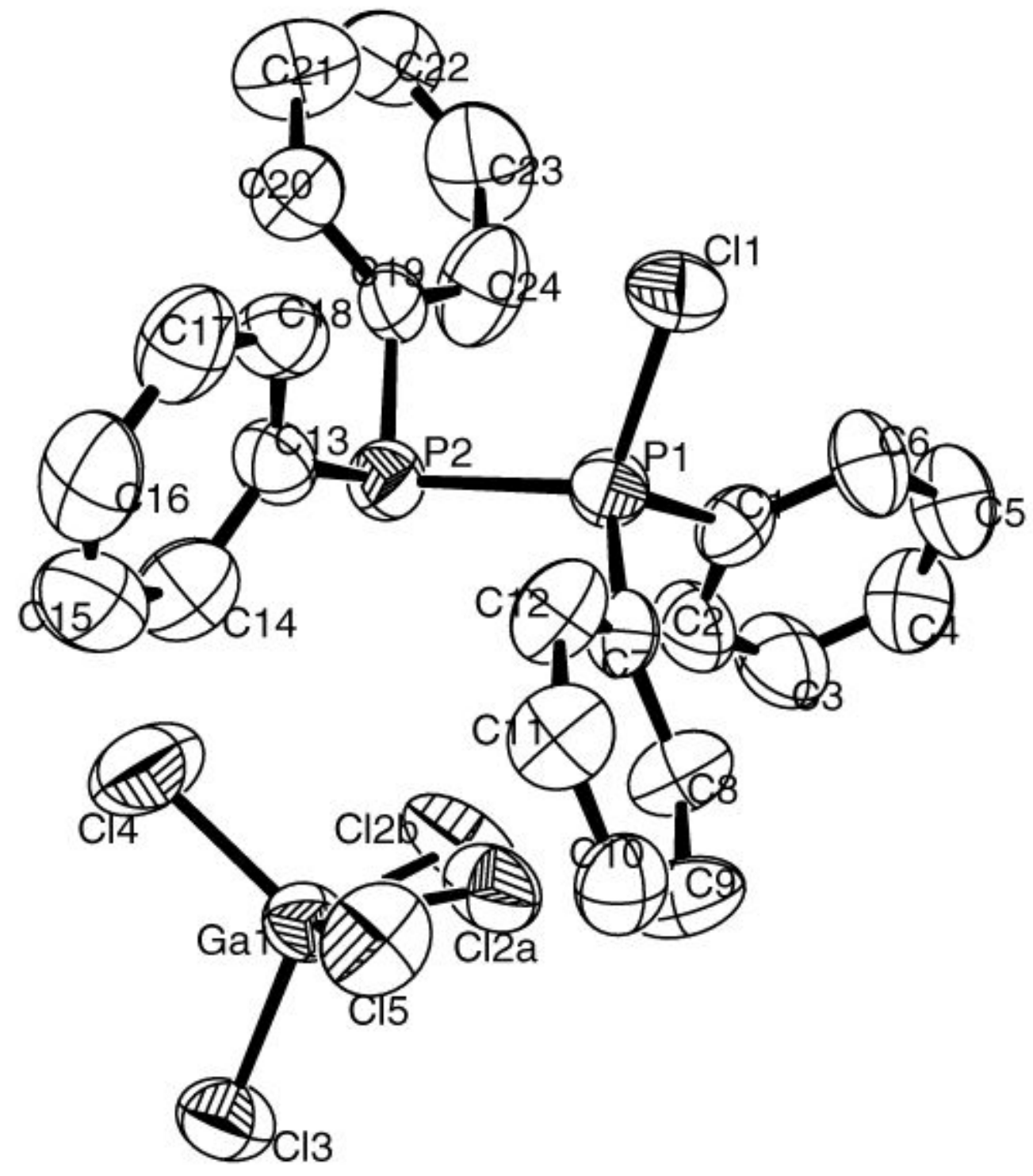

Figure S2: View of $\mathbf{2 a G a C l}_{\mathbf{4}}$ with hydrogen atoms removed. This highlights the complete numbering scheme. 
Table S6. Crystal data and structure refinement for $\mathbf{2} \mathbf{a G a C \mathbf { G } _ { \mathbf { 4 } }}$.

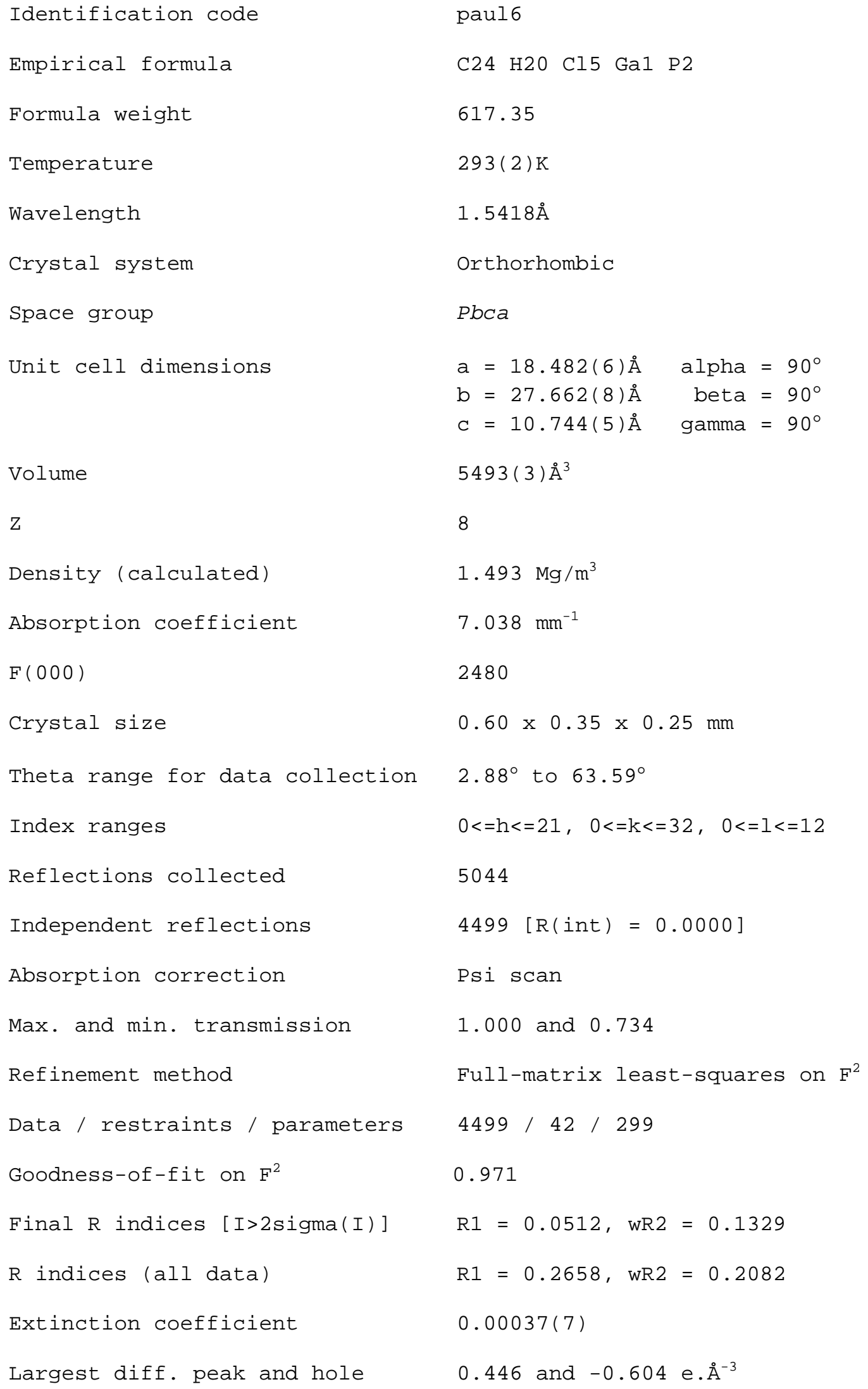


Table S7. Atomic coordinates $\left(\times 10^{4}\right)$ and equivalent isotropic displacement parameters $\left(\AA^{2} \times 10^{3}\right)$ for $\mathbf{2 a G a C l}_{\mathbf{4}}$. U(eq) is defined as one third of the trace of the orthogonalized Uij tensor.

\begin{tabular}{|c|c|c|c|c|}
\hline & $\mathrm{x}$ & $\mathrm{y}$ & z & $\mathrm{U}(\mathrm{eq})$ \\
\hline $\mathrm{Ga}(1)$ & $3321(1)$ & $3912(1)$ & $5829(2)$ & $64(1)$ \\
\hline Cl (1) & $5910(2)$ & $3644(1)$ & $282(3)$ & $73(1)$ \\
\hline $\mathrm{Cl}(2 \mathrm{~A})$ & $4329(5)$ & $4152(4)$ & $6661(13)$ & $92(5)$ \\
\hline $\mathrm{Cl}$ (2B) & $4379(5)$ & $3949(4)$ & $6694(12)$ & $100(5)$ \\
\hline $\mathrm{Cl}(3)$ & $2472(2)$ & $4161(1)$ & $7061(3)$ & $86(1)$ \\
\hline $\mathrm{Cl}(4)$ & $3192(3)$ & $3157(1)$ & $5519(4)$ & $124(2)$ \\
\hline $\mathrm{Cl}(5)$ & $3276(2)$ & $4297(1)$ & $4086(3)$ & $95(1)$ \\
\hline P (1) & $5290(2)$ & $3850(1)$ & 1705 (3) & $53(1)$ \\
\hline P (2) & $4647(2)$ & $3255(1)$ & $2504(3)$ & $56(1)$ \\
\hline$C(1)$ & $5895(6)$ & $4080(4)$ & $2870(10)$ & $54(3)$ \\
\hline$C(2)$ & $5624(7)$ & $4137(5)$ & $4059(12)$ & $82(5)$ \\
\hline$C(3)$ & $6046(8)$ & $4332(6)$ & 4990 (13) & $88(5)$ \\
\hline$C(4)$ & $6732(8)$ & $4479(5)$ & $4719(14)$ & $84(4)$ \\
\hline$C(5)$ & $7004(7)$ & $4418(5)$ & $3541(12)$ & $81(5)$ \\
\hline$C(6)$ & $6586(6)$ & $4228(5)$ & 2610 (11) & $72(4)$ \\
\hline$C(7)$ & $4711(6)$ & $4320(4)$ & $1157(9)$ & $49(3)$ \\
\hline$C(8)$ & $4585(7)$ & $4720(4)$ & $1868(11)$ & $68(4)$ \\
\hline$C(9)$ & $4101(7)$ & $5072(5)$ & $1464(12)$ & $82(4)$ \\
\hline$C(10)$ & $3726(7)$ & $5014(5)$ & $394(12)$ & $65(4)$ \\
\hline C (11) & $3854(7)$ & $4620(5)$ & $-344(12)$ & $70(4)$ \\
\hline$C(12)$ & $4331(7)$ & $4265(5)$ & 52 (11) & $67(4)$ \\
\hline$C(13)$ & $4040(7)$ & $3161(4)$ & $1185(12)$ & $62(4)$ \\
\hline C (14) & $3328(8)$ & 3291 (5) & $1421(14)$ & $80(4)$ \\
\hline C (15) & $2804(8)$ & $3265(6)$ & $488(16)$ & $100(5)$ \\
\hline$C(16)$ & $3015(8)$ & $3115(5)$ & $-650(16)$ & $94(5)$ \\
\hline C (17) & $3720(8)$ & $2993(5)$ & $-905(14)$ & $84(4)$ \\
\hline$C(18)$ & $4255(7)$ & $3012(5)$ & $2(12)$ & $66(4)$ \\
\hline C (19) & $5310(6)$ & $2772(4)$ & $2456(12)$ & $56(3)$ \\
\hline$C(20)$ & $5210(7)$ & $2349(5)$ & $1818(12)$ & $73(4)$ \\
\hline C (21) & $5691(9)$ & $1962(6)$ & $1922(14)$ & $96(5)$ \\
\hline C (22) & $6283(9)$ & $1993(6)$ & $2693(16)$ & $100(6)$ \\
\hline C (23) & $6394(8)$ & $2411(7)$ & $3338(17)$ & $117(7)$ \\
\hline C (24) & $5902(8)$ & $2797(6)$ & $3245(14)$ & $102(5)$ \\
\hline
\end{tabular}


Table S8. Bond lengths $[\AA]$ for $\mathbf{2} \mathbf{a G a C l} \mathbf{G}_{\mathbf{4}}$.

\begin{tabular}{|c|c|}
\hline $\mathrm{Ga}(1)-\mathrm{Cl}(4)$ & $2.128(4)$ \\
\hline $\mathrm{Ga}(1)-\mathrm{Cl}(5)$ & $2.156(4)$ \\
\hline $\mathrm{Ga}(1)-\mathrm{Cl}(2 \mathrm{~B})$ & $2.167(8)$ \\
\hline $\mathrm{Ga}(1)-\mathrm{Cl}(3)$ & $2.167(4)$ \\
\hline $\mathrm{Ga}(1)-\mathrm{Cl}(2 \mathrm{~A})$ & $2.170(8)$ \\
\hline $\mathrm{Cl}(1)-\mathrm{P}(1)$ & $1.993(4)$ \\
\hline $\mathrm{Cl}(2 \mathrm{~A})-\mathrm{Cl}(2 \mathrm{~B})$ & $0.57(2)$ \\
\hline$P(1)-C(7)$ & $1.783(11)$ \\
\hline$P(1)-C(1)$ & $1.795(12)$ \\
\hline$P(1)-P(2)$ & $2.205(4)$ \\
\hline$P(2)-C(19)$ & $1.813(12)$ \\
\hline$P(2)-C(13)$ & $1.826(13)$ \\
\hline$C(1)-C(6)$ & $1.370(11)$ \\
\hline$C(1)-C(2)$ & $1.382(12)$ \\
\hline$C(2)-C(3)$ & $1.379(12)$ \\
\hline $\mathrm{C}(2)-\mathrm{H}(2)$ & 0.9300 \\
\hline$C(3)-C(4)$ & $1.363(12)$ \\
\hline $\mathrm{C}(3)-\mathrm{H}(3)$ & 0.9300 \\
\hline$C(4)-C(5)$ & $1.373(12)$ \\
\hline $\mathrm{C}(4)-\mathrm{H}(4)$ & 0.9300 \\
\hline$C(5)-C(6)$ & $1.369(12)$ \\
\hline $\mathrm{C}(5)-\mathrm{H}(5)$ & 0.9300 \\
\hline $\mathrm{C}(6)-\mathrm{H}(6)$ & 0.9300 \\
\hline$C(7)-C(8)$ & $1.365(11)$ \\
\hline$C(7)-C(12)$ & $1.388(11)$ \\
\hline$C(8)-C(9)$ & $1.393(12)$ \\
\hline $\mathrm{C}(8)-\mathrm{H}(8)$ & 0.9300 \\
\hline$C(9)-C(10)$ & $1.352(11)$ \\
\hline $\mathrm{C}(9)-\mathrm{H}(9)$ & 0.9300 \\
\hline$C(10)-C(11)$ & $1.369(12)$ \\
\hline $\mathrm{C}(10)-\mathrm{H}(10)$ & 0.9300 \\
\hline$C(11)-C(12)$ & $1.384(12)$ \\
\hline $\mathrm{C}(11)-\mathrm{H}(11)$ & 0.9300 \\
\hline $\mathrm{C}(12)-\mathrm{H}(12)$ & 0.9300 \\
\hline$C(13)-C(14)$ & $1.388(12)$ \\
\hline$C(13)-C(18)$ & $1.394(12)$ \\
\hline$C(14)-C(15)$ & $1.396(13)$ \\
\hline $\mathrm{C}(14)-\mathrm{H}(14)$ & 0.9300 \\
\hline$C(15)-C(16)$ & $1.350(13)$ \\
\hline $\mathrm{C}(15)-\mathrm{H}(15)$ & 0.9300 \\
\hline$C(16)-C(17)$ & $1.373(13)$ \\
\hline $\mathrm{C}(16)-\mathrm{H}(16)$ & 0.9300 \\
\hline$C(17)-C(18)$ & $1.389(12)$ \\
\hline $\mathrm{C}(17)-\mathrm{H}(17)$ & 0.9300 \\
\hline $\mathrm{C}(18)-\mathrm{H}(18)$ & 0.9300 \\
\hline$C(19)-C(20)$ & $1.370(12)$ \\
\hline$C(19)-C(24)$ & $1.384(12)$ \\
\hline$C(20)-C(21)$ & $1.395(12)$ \\
\hline $\mathrm{C}(20)-\mathrm{H}(20)$ & 0.9300 \\
\hline$C(21)-C(22)$ & $1.375(13)$ \\
\hline $\mathrm{C}(21)-\mathrm{H}(21)$ & 0.9300 \\
\hline$C(22)-C(23)$ & $1.365(13)$ \\
\hline $\mathrm{C}(22)-\mathrm{H}(22)$ & 0.9300 \\
\hline
\end{tabular}



$C(23)-C(24)$
$1.406(13)$
$\mathrm{C}(23)-\mathrm{H}(23)$
0.9300
$\mathrm{C}(24)-\mathrm{H}(24)$
0.9300 
Table S9. Bond angles $\left[^{\circ}\right]$ for $\mathbf{2} \mathbf{a G a C l} \mathbf{H}_{\mathbf{4}}$.

\begin{tabular}{|c|c|}
\hline $\mathrm{Cl}(4)-\mathrm{Ga}(1)-\mathrm{Cl}(5)$ & $110.2(2)$ \\
\hline $\mathrm{Cl}(4)-\mathrm{Ga}(1)-\mathrm{Cl}(2 \mathrm{~B})$ & $102.5(3)$ \\
\hline $\mathrm{Cl}(5)-\mathrm{Ga}(1)-\mathrm{Cl}(2 \mathrm{~B})$ & $112.6(4)$ \\
\hline $\mathrm{Cl}(4)-\mathrm{Ga}(1)-\mathrm{Cl}(3)$ & $109.1(2)$ \\
\hline $\mathrm{Cl}(5)-\mathrm{Ga}(1)-\mathrm{Cl}(3)$ & $110.2(2)$ \\
\hline $\mathrm{Cl}(2 \mathrm{~B})-\mathrm{Ga}(1)-\mathrm{Cl}(3)$ & $112.1(4)$ \\
\hline $\mathrm{Cl}(4)-\mathrm{Ga}(1)-\mathrm{Cl}(2 \mathrm{~A})$ & $117.5(3)$ \\
\hline $\mathrm{Cl}(5)-\mathrm{Ga}(1)-\mathrm{Cl}(2 \mathrm{~A})$ & $103.9(4)$ \\
\hline $\mathrm{Cl}(2 \mathrm{~B})-\mathrm{Ga}(1)-\mathrm{Cl}(2 \mathrm{~A})$ & $15.1(5)$ \\
\hline $\mathrm{Cl}(3)-\mathrm{Ga}(1)-\mathrm{Cl}(2 \mathrm{~A})$ & $105.8(4)$ \\
\hline $\mathrm{Cl}(2 \mathrm{~B})-\mathrm{Cl}(2 \mathrm{~A})-\mathrm{Ga}(1)$ & $82.2(13)$ \\
\hline $\mathrm{Cl}(2 \mathrm{~A})-\mathrm{Cl}(2 \mathrm{~B})-\mathrm{Ga}(1)$ & $82.7(13)$ \\
\hline $\mathrm{C}(7)-\mathrm{P}(1)-\mathrm{C}(1)$ & $110.2(5)$ \\
\hline $\mathrm{C}(7)-\mathrm{P}(1)-\mathrm{Cl}(1)$ & $107.4(4)$ \\
\hline $\mathrm{C}(1)-\mathrm{P}(1)-\mathrm{Cl}(1)$ & $106.2(4)$ \\
\hline $\mathrm{C}(7)-\mathrm{P}(1)-\mathrm{P}(2)$ & $110.5(4)$ \\
\hline $\mathrm{C}(1)-\mathrm{P}(1)-\mathrm{P}(2)$ & $109.2(4)$ \\
\hline $\mathrm{Cl}(1)-\mathrm{P}(1)-\mathrm{P}(2)$ & $113.3(2)$ \\
\hline$C(19)-P(2)-C(13)$ & $106.8(6)$ \\
\hline $\mathrm{C}(19)-\mathrm{P}(2)-\mathrm{P}(1)$ & $100.0(4)$ \\
\hline$C(13)-P(2)-P(1)$ & $97.7(4)$ \\
\hline$C(6)-C(1)-C(2)$ & $119.5(11)$ \\
\hline$C(6)-C(1)-P(1)$ & $123.0(9)$ \\
\hline $\mathrm{C}(2)-\mathrm{C}(1)-\mathrm{P}(1)$ & $117.3(10)$ \\
\hline$C(3)-C(2)-C(1)$ & $120.7(12)$ \\
\hline $\mathrm{C}(3)-\mathrm{C}(2)-\mathrm{H}(2)$ & 119.7 \\
\hline $\mathrm{C}(1)-\mathrm{C}(2)-\mathrm{H}(2)$ & 119.7 \\
\hline$C(4)-C(3)-C(2)$ & $119.2(13)$ \\
\hline $\mathrm{C}(4)-\mathrm{C}(3)-\mathrm{H}(3)$ & 120.4 \\
\hline $\mathrm{C}(2)-\mathrm{C}(3)-\mathrm{H}(3)$ & 120.4 \\
\hline$C(3)-C(4)-C(5)$ & $120.1(14)$ \\
\hline $\mathrm{C}(3)-\mathrm{C}(4)-\mathrm{H}(4)$ & 119.9 \\
\hline $\mathrm{C}(5)-\mathrm{C}(4)-\mathrm{H}(4)$ & 119.9 \\
\hline$C(6)-C(5)-C(4)$ & $120.9(13)$ \\
\hline $\mathrm{C}(6)-\mathrm{C}(5)-\mathrm{H}(5)$ & 119.6 \\
\hline $\mathrm{C}(4)-\mathrm{C}(5)-\mathrm{H}(5)$ & 119.6 \\
\hline$C(5)-C(6)-C(1)$ & $119.5(12)$ \\
\hline $\mathrm{C}(5)-\mathrm{C}(6)-\mathrm{H}(6)$ & 120.3 \\
\hline $\mathrm{C}(1)-\mathrm{C}(6)-\mathrm{H}(6)$ & 120.3 \\
\hline$C(8)-C(7)-C(12)$ & $118.7(11)$ \\
\hline $\mathrm{C}(8)-\mathrm{C}(7)-\mathrm{P}(1)$ & $120.5(8)$ \\
\hline$C(12)-C(7)-P(1)$ & $120.5(9)$ \\
\hline$C(7)-C(8)-C(9)$ & $120.2(12)$ \\
\hline $\mathrm{C}(7)-\mathrm{C}(8)-\mathrm{H}(8)$ & 119.9 \\
\hline $\mathrm{C}(9)-\mathrm{C}(8)-\mathrm{H}(8)$ & 119.9 \\
\hline$C(10)-C(9)-C(8)$ & $120.7(13)$ \\
\hline $\mathrm{C}(10)-\mathrm{C}(9)-\mathrm{H}(9)$ & 119.6 \\
\hline $\mathrm{C}(8)-\mathrm{C}(9)-\mathrm{H}(9)$ & 119.6 \\
\hline$C(9)-C(10)-C(11)$ & $119.9(13)$ \\
\hline $\mathrm{C}(9)-\mathrm{C}(10)-\mathrm{H}(10)$ & 120.0 \\
\hline $\mathrm{C}(11)-\mathrm{C}(10)-\mathrm{H}(10)$ & 120.0 \\
\hline$C(10)-C(11)-C(12)$ & $119.8(12)$ \\
\hline $\mathrm{C}(10)-\mathrm{C}(11)-\mathrm{H}(11)$ & 120.1 \\
\hline
\end{tabular}




\begin{tabular}{|c|c|}
\hline $\mathrm{C}(12)-\mathrm{C}(11)-\mathrm{H}(11)$ & 120.1 \\
\hline$C(11)-C(12)-C(7)$ & $120.6(12)$ \\
\hline $\mathrm{C}(11)-\mathrm{C}(12)-\mathrm{H}(12)$ & 119.7 \\
\hline $\mathrm{C}(7)-\mathrm{C}(12)-\mathrm{H}(12)$ & 119.7 \\
\hline$C(14)-C(13)-C(18)$ & $120.9(12)$ \\
\hline$C(14)-C(13)-P(2)$ & $113.8(10)$ \\
\hline$C(18)-C(13)-P(2)$ & $125.1(10)$ \\
\hline$C(13)-C(14)-C(15)$ & $120.9(14)$ \\
\hline $\mathrm{C}(13)-\mathrm{C}(14)-\mathrm{H}(14)$ & 119.5 \\
\hline $\mathrm{C}(15)-\mathrm{C}(14)-\mathrm{H}(14)$ & 119.5 \\
\hline$C(16)-C(15)-C(14)$ & $118(2)$ \\
\hline $\mathrm{C}(16)-\mathrm{C}(15)-\mathrm{H}(15)$ & 121.1 \\
\hline $\mathrm{C}(14)-\mathrm{C}(15)-\mathrm{H}(15)$ & 121.1 \\
\hline$C(15)-C(16)-C(17)$ & $122(2)$ \\
\hline $\mathrm{C}(15)-\mathrm{C}(16)-\mathrm{H}(16)$ & 119.0 \\
\hline $\mathrm{C}(17)-\mathrm{C}(16)-\mathrm{H}(16)$ & 119.0 \\
\hline$C(16)-C(17)-C(18)$ & $121.8(14)$ \\
\hline $\mathrm{C}(16)-\mathrm{C}(17)-\mathrm{H}(17)$ & 119.1 \\
\hline $\mathrm{C}(18)-\mathrm{C}(17)-\mathrm{H}(17)$ & 119.1 \\
\hline$C(17)-C(18)-C(13)$ & $116.6(12)$ \\
\hline $\mathrm{C}(17)-\mathrm{C}(18)-\mathrm{H}(18)$ & 121.7 \\
\hline $\mathrm{C}(13)-\mathrm{C}(18)-\mathrm{H}(18)$ & 121.7 \\
\hline$C(20)-C(19)-C(24)$ & $117.0(12)$ \\
\hline$C(20)-C(19)-P(2)$ & $123.5(10)$ \\
\hline$C(24)-C(19)-P(2)$ & $118.7(11)$ \\
\hline$C(19)-C(20)-C(21)$ & $122.0(13)$ \\
\hline $\mathrm{C}(19)-\mathrm{C}(20)-\mathrm{H}(20)$ & 119.0 \\
\hline$C(21)-C(20)-H(20)$ & 119.0 \\
\hline$C(22)-C(21)-C(20)$ & $121(2)$ \\
\hline $\mathrm{C}(22)-\mathrm{C}(21)-\mathrm{H}(21)$ & 119.7 \\
\hline $\mathrm{C}(20)-\mathrm{C}(21)-\mathrm{H}(21)$ & 119.7 \\
\hline$C(23)-C(22)-C(21)$ & $119(2)$ \\
\hline $\mathrm{C}(23)-\mathrm{C}(22)-\mathrm{H}(22)$ & 120.7 \\
\hline $\mathrm{C}(21)-\mathrm{C}(22)-\mathrm{H}(22)$ & 120.7 \\
\hline$C(22)-C(23)-C(24)$ & $121(2)$ \\
\hline $\mathrm{C}(22)-\mathrm{C}(23)-\mathrm{H}(23)$ & 119.7 \\
\hline $\mathrm{C}(24)-\mathrm{C}(23)-\mathrm{H}(23)$ & 119.7 \\
\hline$C(19)-C(24)-C(23)$ & $121.2(14)$ \\
\hline $\mathrm{C}(19)-\mathrm{C}(24)-\mathrm{H}(24)$ & 119.4 \\
\hline $\mathrm{C}(23)-\mathrm{C}(24)-\mathrm{H}(24)$ & 119.4 \\
\hline
\end{tabular}


Table s10. Selected torsion angles $\left[{ }^{\circ}\right]$ for $\mathbf{2} \mathbf{a G a C l}_{\mathbf{4}}$.

\begin{tabular}{|c|c|c|}
\hline-5 & 2 & Cl4 - Ga1 - \\
\hline-127.08 & $(2.61)$ & $\mathrm{Cl} 5-\mathrm{Ga} 1-\mathrm{Cl} 2 \mathrm{~A}$ \\
\hline 116.86 & $(2.61)$ & $\mathrm{Cl} 3-\mathrm{Ga} 1$ \\
\hline 175.31 & $(2.60)$ & $\mathrm{Cl} 4-\mathrm{Ga} 1-\mathrm{Cl} 2 \mathrm{~B}$ \\
\hline 57.02 & $(2.75)$ & $-\mathrm{Cl2B}$ \\
\hline-67.89 & $(2.72)$ & $\mathrm{Cl} 3-\mathrm{Ga} 1$ \\
\hline 160 . & $(0.58)$ & $\mathrm{C} 7-\mathrm{P} 1-\mathrm{P} 2-\mathrm{C} 19$ \\
\hline-77 & $(0.61)$ & $\mathrm{C} 1-\mathrm{P} 1-\mathrm{P} 2-\mathrm{C} 19$ \\
\hline 40 & $(0.49)$ & $\mathrm{Cl1}-\mathrm{P} 1-\mathrm{P} 2-\mathrm{C} 19$ \\
\hline 52. & $(0.58)$ & $\mathrm{C} 7-\mathrm{P} 1-\mathrm{P} 2-\mathrm{C} 13$ \\
\hline 173.63 & $(0.60)$ & $\mathrm{C} 1-\mathrm{P} 1-\mathrm{P} 2-\mathrm{C} 13$ \\
\hline-68.32 & $(0.46)$ & $\mathrm{Cl1}-\mathrm{P} 1-\mathrm{P} 2-\mathrm{C} 13$ \\
\hline-96.10 & $(1.16)$ & $\mathrm{C} 7-\mathrm{P} 1-\mathrm{C} 1-\mathrm{C} 6$ \\
\hline 19.95 & $(1.20)$ & $\mathrm{C} 11-\mathrm{P} 1-\mathrm{C} 1-\mathrm{C} 6$ \\
\hline 142.38 & $(1.03)$ & $\mathrm{P} 2-\mathrm{P} 1-\mathrm{C} 1-\mathrm{C} 6$ \\
\hline 79.22 & $(1.17)$ & $\mathrm{C} 7-\mathrm{P} 1-\mathrm{C} 1-\mathrm{C} 2$ \\
\hline$-164 \cdot 72$ & $(1.02)$ & $\mathrm{C} 11-\mathrm{P} 1-\mathrm{C} 1-\mathrm{C} 2$ \\
\hline$-42 \cdot 30$ & $(1.18)$ & $\mathrm{P} 2-\mathrm{P} 1-\mathrm{C} 1-\mathrm{C} 2$ \\
\hline-1.61 & $(2.26)$ & $\mathrm{C} 6-\mathrm{C} 1-\mathrm{C} 2-\mathrm{C} 3$ \\
\hline-177.11 & $(1.14)$ & $\mathrm{P} 1-\mathrm{C} 1-\mathrm{C} 2-\mathrm{C} 3$ \\
\hline 1.68 & $(2.39)$ & $\mathrm{C} 1-\mathrm{C} 2-\mathrm{C} 3-\mathrm{C} 4$ \\
\hline-2.23 & $(2.38)$ & $\mathrm{C} 2-\mathrm{C} 3-\mathrm{C} 4-\mathrm{C} 5$ \\
\hline 2.75 & $(2.36)$ & $\mathrm{C} 3-\mathrm{C} 4-\mathrm{C} 5-\mathrm{C} 6$ \\
\hline-2.65 & $(2.20)$ & $\mathrm{C} 4-\mathrm{C} 5-\mathrm{C} 6-\mathrm{C} 1$ \\
\hline 2.07 & $(2.10)$ & $\mathrm{C} 2-\mathrm{C} 1-\mathrm{C} 6-\mathrm{C} 5$ \\
\hline 177.29 & $(1.01)$ & $\mathrm{P} 1-\mathrm{C} 1-\mathrm{C} 6-\mathrm{C} 5$ \\
\hline-22.40 & $(1.15)$ & $\mathrm{C} 1-\mathrm{P} 1-\mathrm{C} 7-\mathrm{C} 8$ \\
\hline-137.65 & $(0.92)$ & $\mathrm{Cl1}-\mathrm{P} 1-\mathrm{C} 7-\mathrm{C} 8$ \\
\hline 98.38 & $(0.98)$ & $\mathrm{P} 2-\mathrm{P} 1-\mathrm{C} 7-\mathrm{C} 8$ \\
\hline 163.43 & $(0.92)$ & $\mathrm{C} 1-\mathrm{P} 1-\mathrm{C} 7-\mathrm{C} 12$ \\
\hline 48.18 & $(0.99)$ & $\mathrm{C} 11-\mathrm{P} 1-\mathrm{C} 7-\mathrm{C} 12$ \\
\hline-75.79 & $(0.95)$ & $\mathrm{P} 2-\mathrm{P} 1-\mathrm{C} 7-\mathrm{C} 12$ \\
\hline-2.15 & $(1.87)$ & $\mathrm{C} 12-\mathrm{C} 7-\mathrm{C} 8-\mathrm{C} 9$ \\
\hline-176.42 & $(1.01)$ & $\mathrm{P} 1-\mathrm{C} 7-\mathrm{C} 8-\mathrm{C} 9$ \\
\hline 3.05 & $(2.12)$ & $\mathrm{C} 7-\mathrm{C} 8-\mathrm{C} 9-\mathrm{C} 10$ \\
\hline-4.23 & $(2.10)$ & $\mathrm{C} 8-\mathrm{C} 9-\mathrm{C} 10-\mathrm{C} 11$ \\
\hline 4.57 & $(2.01)$ & $\mathrm{C} 9-\mathrm{C} 10-\mathrm{C} 11-\mathrm{C} 12$ \\
\hline-3.74 & $(1.95)$ & $\mathrm{C} 10-\mathrm{C} 11-\mathrm{C} 12-\mathrm{C} 7$ \\
\hline 2.53 & $1.80)$ & $\mathrm{C} 8-\mathrm{C} 7-\mathrm{C} 12-\mathrm{C} 11$ \\
\hline 176.80 & $0.96)$ & $\mathrm{P} 1-\mathrm{C} 7-\mathrm{C} 12-\mathrm{C} 11$ \\
\hline 145.36 & $(0.99)$ & $\mathrm{C} 19-\mathrm{P} 2-\mathrm{C} 13-\mathrm{C} 14$ \\
\hline-111.68 & $(0.97)$ & $\mathrm{P} 1-\mathrm{P} 2-\mathrm{C} 13-\mathrm{C} 14$ \\
\hline-39.73 & $1.23)$ & $\mathrm{C} 19-\mathrm{P} 2-\mathrm{C} 13-\mathrm{C} 18$ \\
\hline 63.24 & $1.12)$ & $\mathrm{P} 1-\mathrm{P} 2-\mathrm{C} 13-\mathrm{C} 18$ \\
\hline 1.46 & $(2.09)$ & $\mathrm{C} 18-\mathrm{C} 13-\mathrm{C} 14-\mathrm{C} 15$ \\
\hline 176.62 & $(1.11)$ & $\mathrm{P} 2-\mathrm{C} 13-\mathrm{C} 14-\mathrm{C} 15$ \\
\hline-0.58 & $2.30)$ & $\mathrm{C} 13-\mathrm{C} 14-\mathrm{C} 15-\mathrm{C} 16$ \\
\hline-0.56 & $2.48)$ & $\mathrm{C} 14-\mathrm{C} 15-\mathrm{C} 16-\mathrm{C} 17$ \\
\hline 0.84 & $2.37)$ & $\mathrm{C} 15-\mathrm{C} 16-\mathrm{C} 17$ \\
\hline 0.04 & $1.96)$ & $\mathrm{C} 16-\mathrm{C} 17-\mathrm{C} 18-\mathrm{C} 13$ \\
\hline-1.16 & $1.91)$ & $\mathrm{C} 14-\mathrm{C} 13-\mathrm{C} 18-\mathrm{C} 17$ \\
\hline-175.74 & $(0.93)$ & $\mathrm{P} 2-\mathrm{C} 13-\mathrm{C} 18-\mathrm{C} 17$ \\
\hline-19.50 & $(1.26)$ & $\mathrm{C} 13-\mathrm{P} 2-\mathrm{C} 19-\mathrm{C} 20$ \\
\hline-120.81 & $(1.06)$ & $\mathrm{P} 1-\mathrm{P} 2-\mathrm{C} 19-\mathrm{C} 20$ \\
\hline
\end{tabular}




$$
\begin{array}{rll}
170.49 & (1.03) & \mathrm{C} 13-\mathrm{P} 2-\mathrm{C} 19-\mathrm{C} 24 \\
69.18 & (1.07) & \mathrm{P} 1-\mathrm{P} 2-\mathrm{C} 19-\mathrm{C} 24 \\
-1.90 & (2.05) & \mathrm{C} 24-\mathrm{C} 19-\mathrm{C} 20-\mathrm{C} 21 \\
-172.07 & (1.07) & \mathrm{P} 2-\mathrm{C} 19-\mathrm{C} 20-\mathrm{C} 21 \\
0.95 & (2.33) & \mathrm{C} 19-\mathrm{C} 20-\mathrm{C} 21-\mathrm{C} 22 \\
-0.97 & (2.47) & \mathrm{C} 20-\mathrm{C} 21-\mathrm{C} 22-\mathrm{C} 23 \\
2.02 & (2.67) & \mathrm{C} 21-\mathrm{C} 22-\mathrm{C} 23-\mathrm{C} 24 \\
2.94 & (2.20) & \mathrm{C} 20-\mathrm{C} 19-\mathrm{C} 24-\mathrm{C} 23 \\
173.60 & (1.27) & \mathrm{P} 2-\mathrm{C} 19-\mathrm{C} 24-\mathrm{C} 23 \\
-3.10 & (2.67) & \mathrm{C} 22-\mathrm{C} 23-\mathrm{C} 24-\mathrm{C} 19
\end{array}
$$


Table S11. Selected contact distances [A] and angles [ $\left.{ }^{\circ}\right]$ and best plane calculations for $\mathbf{2} \mathbf{a G a C l}_{\mathbf{4}}$.

Distance $\mathrm{Cl} \ldots \mathrm{Cl}$

$$
0.5706(0.0173) \quad \mathrm{C} 12 \mathrm{~A}-\mathrm{C} 12 \mathrm{~B}
$$

Distance $\mathrm{Cl}$..C

$$
\begin{aligned}
& 3.3639(0.0150) \quad \mathrm{C} 11-\mathrm{C} 22 \_\$ 1 \\
& 3.6990(0.0179) \quad \mathrm{C} 11-\mathrm{C} 23 \_\$ 1 \\
& 3.6805(0.0189) \quad \mathrm{Cl} 2 \mathrm{~A}-\mathrm{C} 2 \\
& 3.6799(0.0190) \quad \mathrm{Cl} 2 \mathrm{~A}-\mathrm{C} 3 \\
& 3.5772(0.0191) \quad \text { C12A - C11_\$2 } \\
& 3.6567(0.0180) \quad \mathrm{Cl} 2 \mathrm{~A}-\mathrm{C} 12 \_\$ 2 \\
& 3.6849(0.0180) \quad \mathrm{Cl} 2 \mathrm{~B}-\mathrm{C} 2 \\
& 3.7366(0.0187) \quad \mathrm{Cl} 2 \mathrm{~B}-\mathrm{C} 3 \\
& 3.6555(0.0134) \quad \mathrm{Cl3}-\mathrm{C} 9 \_\$ 3 \\
& 3.6495(0.0143) \quad \text { C13 - C10_\$3 } \\
& 3.7494(0.0147) \quad \text { Cl4 - C16_\$4 } \\
& 3.6613(0.0134) \quad \text { C14 - C17_\$4 } \\
& 3.6878(0.0147) \quad \text { C15 - C5_\$5 } \\
& 3.6211(0.0125) \quad \text { C15 - C6_\$5 }
\end{aligned}
$$

Angle $\mathrm{P}-\mathrm{Cl}$.. C

$$
\begin{aligned}
& 154.39(0.34) \\
& 142.94(0.33)
\end{aligned}
$$

Angle $\mathrm{Ga}-\mathrm{Cl} . \mathrm{C}$

$$
\begin{array}{lll}
103.97 & (0.50) & \mathrm{Ga} 1-\mathrm{C} 12 \mathrm{~A}-\mathrm{C} 2 \\
125.48 & (0.57) & \mathrm{Ga} 1-\mathrm{C} 12 \mathrm{~A}-\mathrm{C} 3 \\
105.75 & (0.50) & \mathrm{Ga} 1-\mathrm{C} 12 \mathrm{~A}-\mathrm{C} 11 \_\$ 2 \\
115.96 & (0.55) & \mathrm{Ga} 1-\mathrm{C} 12 \mathrm{~A}-\mathrm{C} 12 \_\$ 2 \\
103.90 & (0.47) & \mathrm{Ga} 1-\mathrm{C} 12 \mathrm{~B}-\mathrm{C} 2 \\
123.17 & (0.53) & \mathrm{Ga} 1-\mathrm{C} 12 \mathrm{~B}-\mathrm{C} 3 \\
130.82 & (0.26) & \mathrm{Ga} 1-\mathrm{C} 13-\mathrm{C} 9 \_\$ 3 \\
109.80 & (0.24) & \mathrm{Ga} 1-\mathrm{C} 13-\mathrm{C} 10 \_\$ 3 \\
169.37 & (0.34) & \mathrm{Ga} 1-\mathrm{C} 14-\mathrm{C} 16 \_\$ 4 \\
152.61 & (0.31) & \mathrm{Ga} 1-\mathrm{C} 14-\mathrm{C} 17 \_\$ 4 \\
137.27 & (0.26) & \mathrm{Ga} 1-\mathrm{C} 15-\mathrm{C} 5 \$ 5 \\
116.41 & (0.25) & \mathrm{Ga} 1-\mathrm{C} 15-\mathrm{C} 6 \_\$ 5
\end{array}
$$

Specified potential hydrogen bonds and other contacts

(with esds except fixed and riding $\mathrm{H}$ )

$\begin{array}{lllll}\mathrm{D}-\mathrm{H} & \mathrm{H} \ldots \mathrm{A} & \mathrm{D} \ldots \mathrm{A} & <(\mathrm{DHA}) & \\ 0.93 & 3.03 & 3.681(19) & 128.0 & \mathrm{C} 2-\mathrm{H} 2 \ldots \mathrm{C} 12 \mathrm{~A} \\ 0.93 & 3.02 & 3.685(18) & 130.1 & \mathrm{C} 2-\mathrm{H} 2 \ldots \mathrm{Cl} \text { 2B } \\ 0.93 & 3.04 & 3.680(19) & 127.0 & \mathrm{C} 3-\mathrm{H} 3 \ldots \mathrm{C} 12 \mathrm{~A} \\ 0.93 & 3.13 & 3.737(19) & 124.7 & \mathrm{C} 3-\mathrm{H} 3 \ldots \mathrm{C} 12 \mathrm{~B} \\ 0.93 & 3.19 & 3.823(15) & 127.1 & \mathrm{C} 4-\mathrm{H} 4 \ldots \mathrm{C} 13 \_\$ 7\end{array}$




\begin{tabular}{|c|c|c|c|c|}
\hline 0.93 & 3.10 & $3.622(15)$ & 116.9 & $\mathrm{C} 4-\mathrm{H} 4 \ldots \mathrm{Cl} 5 \_\$ 6$ \\
\hline 0.93 & 3.08 & $3.688(15)$ & 124.6 & C5-H5...Cl5_\$8 \\
\hline 0.93 & 2.75 & $3.229(13)$ & 112.8 & $\mathrm{C} 6-\mathrm{H} 6 \ldots \mathrm{Cl} 1$ \\
\hline 0.93 & 2.96 & $3.621(12)$ & 129.5 & C6-H6...Cl5_\$8 \\
\hline 0.93 & 3.10 & $3.655(13)$ & 120.2 & C9-H9...Cl3_\$9 \\
\hline 0.93 & 3.06 & $3.649(14)$ & 122.5 & $\mathrm{C} 10-\mathrm{H} 10 \ldots \mathrm{Cl} 3 \_\$ 9$ \\
\hline 0.93 & 2.99 & $3.577(19)$ & 122.9 & C11-H11...Cl2A_\$10 \\
\hline 0.93 & 3.27 & $3.809(18)$ & 119.3 & C11-H11...Cl2B_\$10 \\
\hline 0.93 & 3.13 & $3.989(14)$ & 154.4 & C11-H11...Cl3_\$10 \\
\hline 0.93 & 3.05 & $3.396(13)$ & 103.8 & $\mathrm{C} 12-\mathrm{H} 12 \ldots \mathrm{Cl1}$ \\
\hline 0.93 & 3.16 & $3.657(18)$ & 115.1 & $\mathrm{C} 12-\mathrm{H} 12 \ldots \mathrm{Cl} 2 \mathrm{~A} \_\$ 10$ \\
\hline 0.93 & 3.10 & $3.713(17)$ & 125.5 & $\mathrm{C} 12-\mathrm{H} 12 \ldots \mathrm{Cl} 2 \mathrm{~B} \_\$ 10$ \\
\hline 0.93 & 3.21 & $3.993(15)$ & 143.7 & $\mathrm{C} 14-\mathrm{H} 14 \ldots \mathrm{Cl} 5$ \\
\hline 0.93 & 3.30 & $3.990(15)$ & 133.2 & C17-H17...Cl4_\$10 \\
\hline 0.93 & 2.98 & $3.535(13)$ & 119.8 & $\mathrm{C} 18-\mathrm{H} 18 \ldots \mathrm{Cl} 1$ \\
\hline 0.93 & 2.88 & $3.51(2)$ & 126.0 & $\mathrm{C} 21-\mathrm{H} 21 \ldots \mathrm{Cl} 2 \mathrm{~B} \_\$ 1$ \\
\hline 0.93 & 2.96 & $3.884(16)$ & 173.5 & C22-H22...C13_\$11 \\
\hline 0.93 & 3.13 & $3.875(15)$ & 137.9 & C23-H23...C14_\$11 \\
\hline 0.93 & 3.00 & $3.762(17)$ & 139.6 & C5-H5 ...C10_\$8 \\
\hline 0.93 & 3.06 & $3.80(2)$ & 138.1 & $\mathrm{C} 17-\mathrm{H} 17 \ldots \mathrm{C} 20 \_\$ 1$ \\
\hline 0.93 & 3.03 & $3.74(2)$ & 134.5 & $\mathrm{C} 23-\mathrm{H} 23 \ldots \mathrm{C} 15 \_\$ 8$ \\
\hline 0.93 & 2.57 & $3.422(19)$ & 152.0 & C3-H3 ...H9_\$6 \\
\hline 0.93 & 2.63 & $3.338(17)$ & 133.3 & C10-H10...H6_\$13 \\
\hline 0.93 & 2.59 & $3.50(2)$ & 163.5 & C16-H16...H22_\$12 \\
\hline
\end{tabular}

Least-squares planes ( $\mathrm{x}, \mathrm{y}, \mathrm{z}$ in crystal coordinates) and deviations from them (* indicates atom used to define plane)

$-6.2011(0.0975) x+25.2079(0.0614) y-2.5650(0.0579) z=5.8971$ $(0.0860)$

$\begin{array}{lrll}* & -0.0053 & (0.0099) & \mathrm{C} 1 \\ \star & 0.0040 & (0.0108) & \mathrm{C} 2 \\ \star & -0.0061 & (0.0110) & \mathrm{C} 3 \\ \star & 0.0096 & (0.0108) & \mathrm{C} 4 \\ \star & -0.0110 & (0.0105) & \mathrm{C} 5 \\ \star & 0.0088 & (0.0097) & \mathrm{C} 6 \\ & 0.0895 & (0.0171) & \mathrm{P} 1\end{array}$

Rms deviation of fitted atoms $=0.0079$

$13.5643(0.0566) x+13.2259(0.1259) y-5.1837(0.0457) z=11.4995$ $(0.0522)$

Angle to previous plane (with approximate esd) $=72.26(0.39$ )

$\begin{array}{lrll}* & 0.0048 & (0.0083) & \mathrm{C} 7 \\ \star & -0.0062 & (0.0094) & \mathrm{C} 8 \\ \star & 0.0132 & (0.0099) & \mathrm{C} 9 \\ \star & -0.0187 & (0.0093) & \mathrm{C} 10 \\ \star & 0.0170 & (0.0093) & \mathrm{C} 11\end{array}$




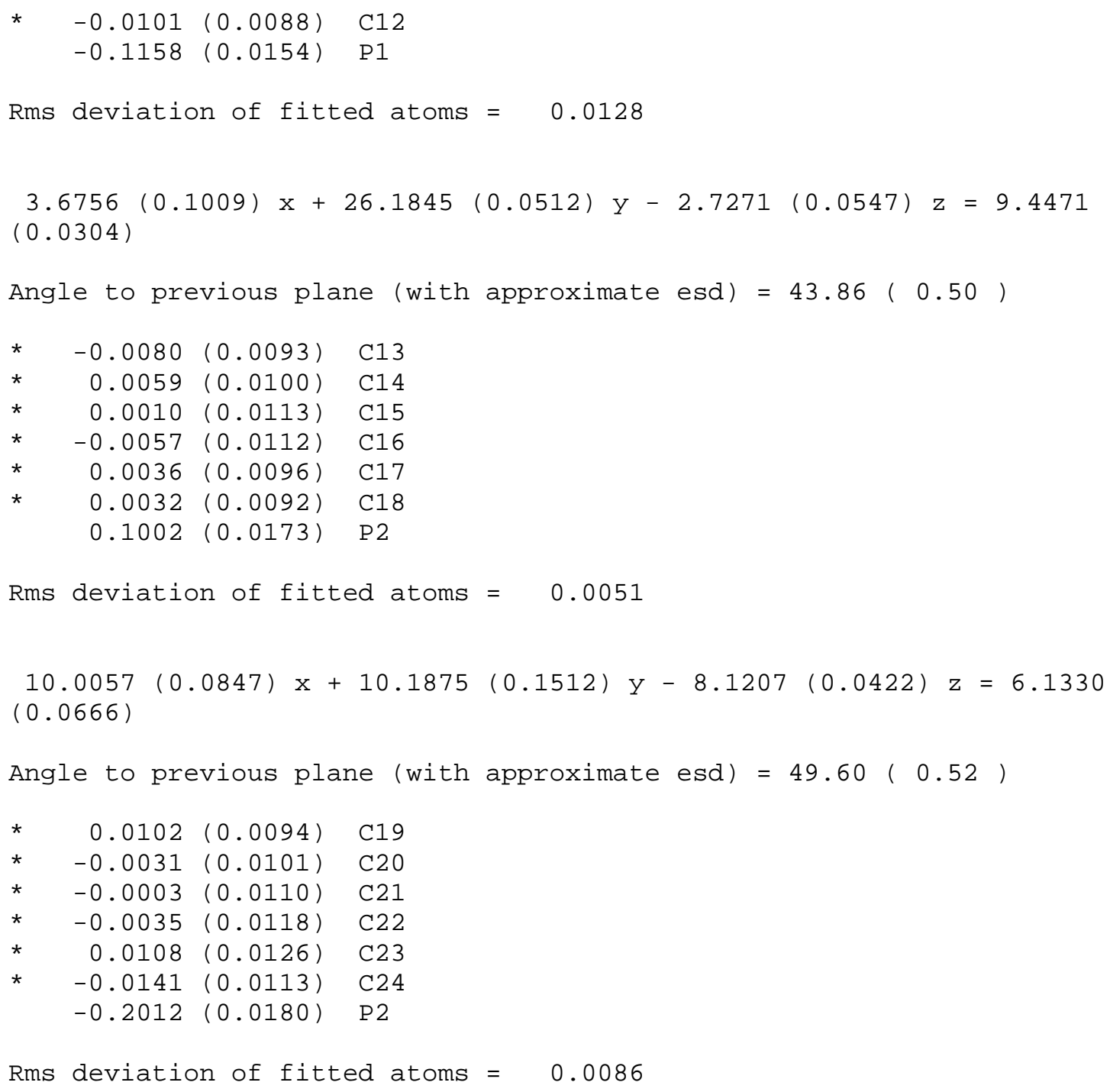


Table S12. Anisotropic displacement parameters $\left(\AA^{2} \times 10^{3}\right)$ for $\mathbf{2 a G a C l}_{4}$. The anisotropic displacement factor exponent takes the form: $-2 \mathrm{pi}^{2}\left[\mathrm{~h}^{2} \mathrm{a} \star^{2} \mathrm{U} 11+\ldots+2 \mathrm{~h} \mathrm{k} a \mathrm{a}^{*} \mathrm{~b} 12\right]$

$\mathrm{U} 12$

$\begin{array}{lllll}\text { U11 } & \text { U22 } & \text { U33 } & \text { U23 }\end{array}$

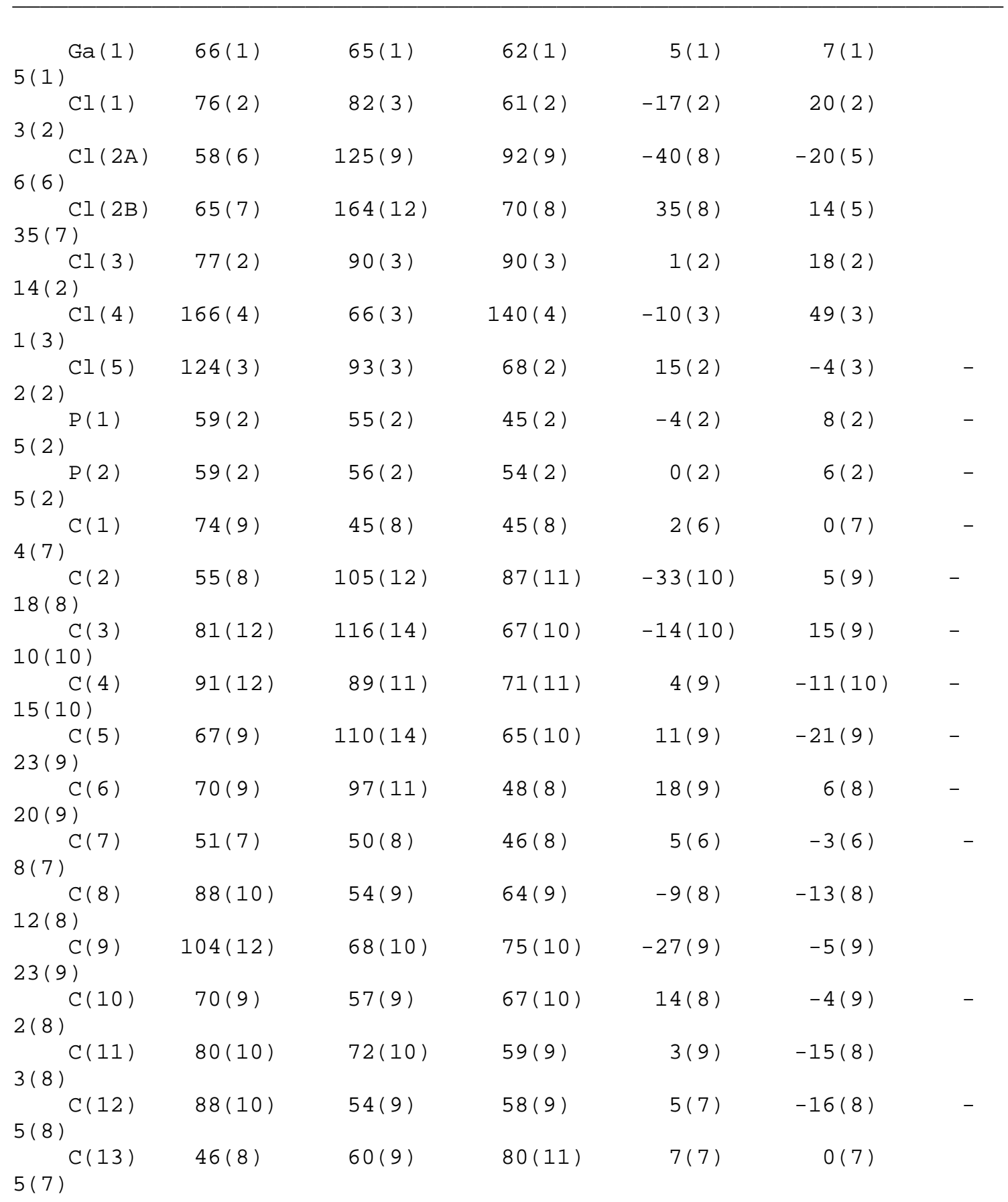




\begin{tabular}{|c|c|c|c|c|c|c|}
\hline $\mathrm{C}(14)$ & $99(12)$ & $68(9)$ & $74(10)$ & $6(8)$ & $-11(10)$ & \\
\hline \multicolumn{7}{|l|}{$5(10)$} \\
\hline$C(15)$ & $74(11)$ & $106(14)$ & $122(15)$ & $-13(13)$ & $-1(12)$ & \\
\hline \multicolumn{7}{|l|}{$15(10)$} \\
\hline $\mathrm{C}(16)$ & $77(11)$ & $88(13)$ & $117(15)$ & $9(11)$ & $-54(11)$ & - \\
\hline \multicolumn{7}{|l|}{$2(9)$} \\
\hline $\mathrm{C}(17)$ & $94(12)$ & $60(10)$ & $98(12)$ & $10(9)$ & $-25(11)$ & - \\
\hline \multicolumn{7}{|l|}{$4(9)$} \\
\hline $\mathrm{C}(18)$ & $67(9)$ & $56(9)$ & $75(10)$ & $4(8)$ & $3(8)$ & \\
\hline \multicolumn{7}{|l|}{$11(8)$} \\
\hline C (19) & $51(8)$ & $60(9)$ & $56(8)$ & $-3(8)$ & $13(7)$ & - \\
\hline \multicolumn{7}{|l|}{$13(7)$} \\
\hline$C(20)$ & $74(10)$ & $69(10)$ & $78(10)$ & $1(9)$ & $-17(9)$ & \\
\hline \multicolumn{7}{|l|}{$5(8)$} \\
\hline $\mathrm{C}(21)$ & $132(15)$ & 88 (12) & $70(10)$ & $15(10)$ & $-19(10)$ & \\
\hline \multicolumn{7}{|l|}{$35(11)$} \\
\hline $\mathrm{C}(22)$ & $110(14)$ & 61 (11) & $128(17)$ & $50(12)$ & $39(13)$ & \\
\hline \multicolumn{7}{|l|}{$28(11)$} \\
\hline $\mathrm{C}(23)$ & $80(12)$ & $104(15)$ & $166(18)$ & $53(16)$ & $-29(12)$ & \\
\hline \multicolumn{7}{|l|}{$26(12)$} \\
\hline $\mathrm{C}(24)$ & $110(13)$ & $89(13)$ & $108(13)$ & $5(11)$ & $-42(11)$ & - \\
\hline $22(11)$ & & & & & & \\
\hline
\end{tabular}


Table S13. Hydrogen coordinates $\left(\mathrm{x} 10^{4}\right)$ and isotropic displacement parameters $\left(\AA^{2} \times 10^{3}\right)$ for $\mathbf{2} \mathbf{a G a C l}_{\mathbf{4}}$.

\begin{tabular}{|c|c|c|c|c|}
\hline & $\mathrm{x}$ & $\mathrm{y}$ & z & $\mathrm{U}(\mathrm{eq})$ \\
\hline $\mathrm{H}(2)$ & 5151 & 4044 & 4232 & 99 \\
\hline H (3) & 5865 & 4362 & 5794 & 106 \\
\hline H (4) & 7015 & 4621 & 5333 & 100 \\
\hline H (5) & 7480 & 4507 & 3373 & 97 \\
\hline $\mathrm{H}(6)$ & 6769 & 4201 & 1806 & 86 \\
\hline H (8) & 4823 & 4757 & 2625 & 82 \\
\hline $\mathrm{H}(9)$ & 4035 & 5351 & 1935 & 99 \\
\hline $\mathrm{H}(10)$ & 3381 & 5241 & 160 & 78 \\
\hline $\mathrm{H}(11)$ & 3623 & 4590 & -1108 & 84 \\
\hline $\mathrm{H}(12)$ & 4397 & 3989 & -427 & 80 \\
\hline $\mathrm{H}(14)$ & 3199 & 3396 & 2212 & 96 \\
\hline H (15) & 2325 & 3350 & 646 & 120 \\
\hline $\mathrm{H}(16)$ & 2672 & 3093 & -1282 & 113 \\
\hline $\mathrm{H}(17)$ & 3842 & 2895 & -1705 & 101 \\
\hline $\mathrm{H}(18)$ & 4732 & 2930 & -172 & 79 \\
\hline $\mathrm{H}(20)$ & 4810 & 2318 & 1300 & 88 \\
\hline $\mathrm{H}(21)$ & 5610 & 1682 & 1465 & 116 \\
\hline $\mathrm{H}(22)$ & 6600 & 1734 & 2773 & 120 \\
\hline H (23) & 6800 & 2441 & 3843 & 140 \\
\hline $\mathrm{H}(24)$ & 5974 & 3073 & 3722 & 123 \\
\hline
\end{tabular}




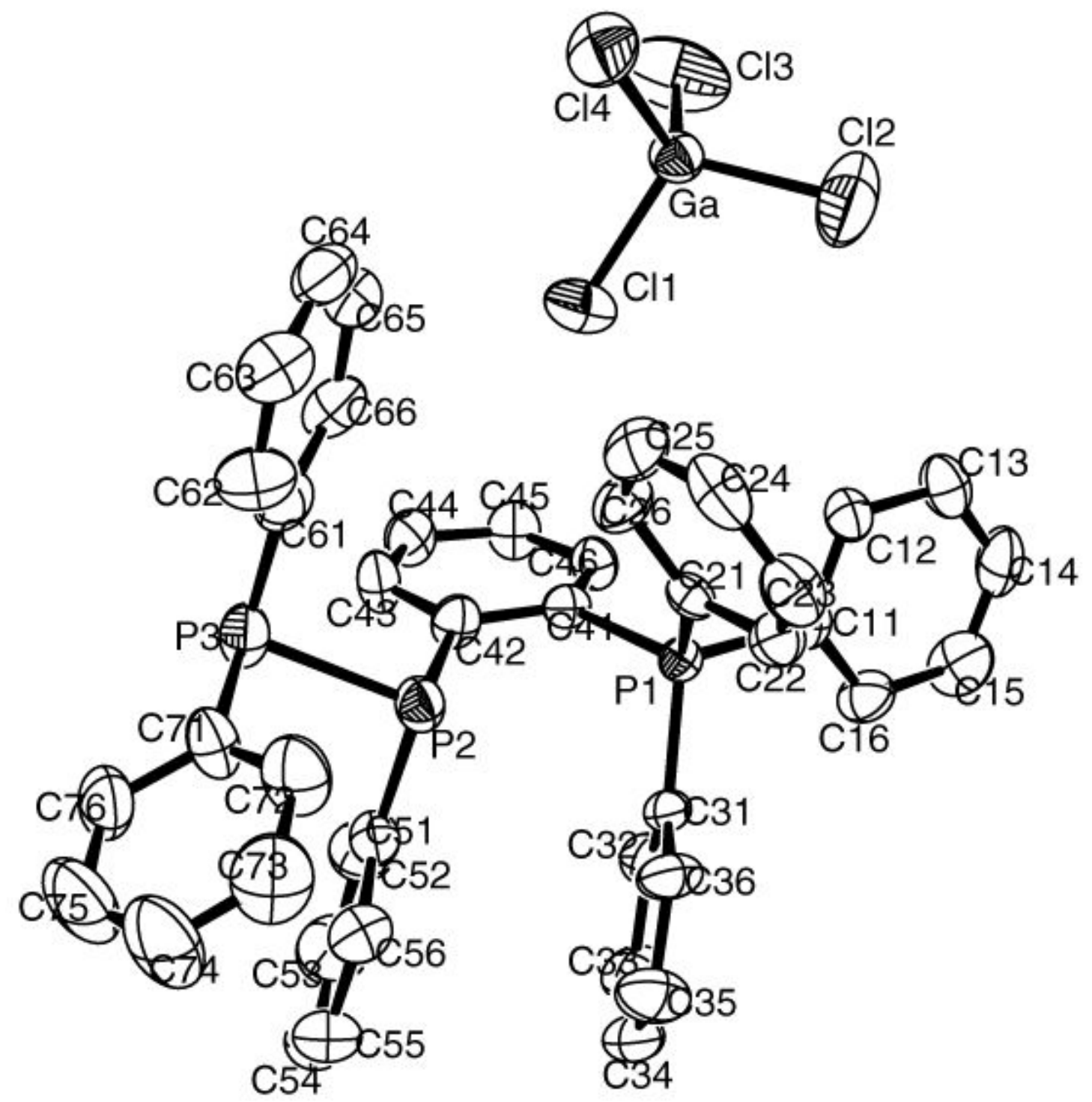

Figuren S3: Solid state structure of $\underline{\mathbf{3}}\left[\mathrm{GaCl}_{4}\right]$ including complete numbering scheme. Ellipsoides are $50 \%$ probability. Hydrogen atoms are removed for clarity and symmetry equivalent atoms are not shown. 


\section{Special Details:}

Refinement of $\mathrm{F}^{\wedge} 2^{\wedge}$ against ALL reflections. The weighted $\mathrm{R}$-factor $\mathrm{wR}$ and goodness of fit $\mathrm{S}$ are based on $\mathrm{F}^{\wedge} 2^{\wedge}$, conventional $\mathrm{R}$-factors $\mathrm{R}$ are based on $\mathrm{F}$, with $\mathrm{F}$ set to zero for negative $\mathrm{F}^{\wedge} 2^{\wedge}$. The threshold expression of $\mathrm{F}^{\wedge} 2^{\wedge}>2 \operatorname{sigma}\left(\mathrm{F}^{\wedge} 2^{\wedge}\right)$ is used only for calculating R-factors(gt) etc. and is not relevant to the choice of reflections for refinement. R-factors based on $\mathrm{F}^{\wedge} 2^{\wedge}$ are statistically about twice as large as those based on $\mathrm{F}$, and $\mathrm{R}$ factors based on ALL data will be even larger.

Although the atoms of the solvent molecules were not included in the refinement, the SQUEEZE routine as implemented in the PLATON program system was used to calculate the contribution of the disordered solvent electron density to the intensity data. A total density of 83 electrons was found in solvent-accessible voids in the unit cell, corresponding to two molecules of $\mathrm{CH} 2 \mathrm{Cl} 2$ (or one-half equivalent of $\mathrm{CH} 2 \mathrm{Cl} 2$ per formula unit of the cation and anion). The refinement proceeded using data from which this contribution had been removed.

References for PLATON/SQUEEZE:

(a) Spek, A. L. Acta Crystallogr. 1990, A46, C34.

(b) PLATON, a multipurpose crystallographic tool. Utrecht University, The Netherlands. A. L. Spek (2002).

(c) van der Sluis, P.; Spek, A. L. Acta Crystallogr. 1990, A46, 194-201. 
Table S14. Crystal data and structure refinement for da10303 1-diphosphino-2-phosphoniumbenze $\left(3 \mathrm{GaCl} 4 \bullet 0.5 \mathrm{CH}_{2} \mathrm{Cl}_{2}\right)$.

Identification code

Empirical formula

Formula weight

Temperature

Wavelength

Crystal system

Space group

Unit cell dimensions

Volume

$\mathrm{Z}$

Density (calculated)

Absorption coefficient

$\mathrm{F}(000)$

Crystal size

Theta range for data collection

Index ranges

Reflections collected

Independent reflections

Completeness to theta $=26.40^{\circ}$

Absorption correction

Max. and min. transmission

Refinement method

Data / restraints / parameters

Goodness-of-fit on $\mathrm{F}^{2}$

Final $\mathrm{R}$ indices $[\mathrm{I}>2 \operatorname{sigma}(\mathrm{I})]$

$\mathrm{R}$ indices (all data)

Largest diff. peak and hole dal0303

C42.50 H35 C15 Ga P3

885.58

193(2) K

$0.71073 \AA$

Monoclinic

P 21/n

$\mathrm{a}=9.8810(10) \AA$

$\alpha=90^{\circ}$.

$\mathrm{b}=14.6051(14) \AA$

$\beta=91.502(2)^{\circ}$.

$\mathrm{c}=28.467(3) \AA$ $\gamma=90^{\circ}$.

4106.7(7) $\AA^{3}$

4

$1.432 \mathrm{Mg} / \mathrm{m}^{3}$

$1.143 \mathrm{~mm}^{-1}$

1804

$0.33 \times 0.20 \times 0.12 \mathrm{~mm}^{3}$

1.43 to $26.40^{\circ}$.

$-9<=\mathrm{h}<=12,-18<=\mathrm{k}<=18,-35<=\mathrm{l}<=35$

20785

$8417[\mathrm{R}(\mathrm{int})=0.0725]$

$99.8 \%$

Semi-empirical from equivalents

0.8751 and 0.7043

Full-matrix least-squares on $\mathrm{F}^{2}$

8417 / 0 / 451

1.024

$\mathrm{R} 1=0.0829, \mathrm{wR} 2=0.2024$

$\mathrm{R} 1=0.1360, \mathrm{wR} 2=0.2272$

2.217 and -0.613 e. $\AA^{-3}$ 
Table S15. Atomic coordinates ( $\left.\mathrm{x} 10^{4}\right)$ and equivalent isotropic displacement parameters $\left(\AA^{2} \times 10^{3}\right)$ for dal0303 1-diphosphino-2-phosphoniumbenze (3GaCl4• $\left.\mathbf{0 . 5} \mathbf{C H}_{\mathbf{2}} \mathbf{C l} \mathbf{l}_{\mathbf{2}}\right)$. U(eq) is defined as one third of the trace of the orthogonalized $\mathrm{U}^{\mathrm{ij}}$ tensor.

\begin{tabular}{|c|c|c|c|c|}
\hline & $\mathrm{x}$ & $\mathrm{y}$ & $\mathrm{z}$ & $\mathrm{U}(\mathrm{eq})$ \\
\hline $\mathrm{Ga}$ & $79(1)$ & $3167(1)$ & $8449(1)$ & $43(1)$ \\
\hline $\mathrm{Cl}(1)$ & $86(2)$ & $2316(2)$ & $7829(1)$ & $78(1)$ \\
\hline $\mathrm{Cl}(2)$ & $-1916(2)$ & $3744(2)$ & $8538(1)$ & $108(1)$ \\
\hline $\mathrm{Cl}(3)$ & $574(4)$ & $2279(2)$ & $9036(1)$ & $112(1)$ \\
\hline $\mathrm{Cl}(4)$ & $1534(2)$ & $4256(1)$ & $8397(1)$ & $72(1)$ \\
\hline $\mathrm{P}(1)$ & $-3165(1)$ & $2324(1)$ & $6666(1)$ & $28(1)$ \\
\hline $\mathrm{P}(2)$ & $-754(2)$ & $2233(1)$ & $5921(1)$ & $42(1)$ \\
\hline $\mathrm{P}(3)$ & $1441(2)$ & 2411(1) & $5759(1)$ & $47(1)$ \\
\hline $\mathrm{C}(11)$ & $-4333(6)$ & 2186(4) & $7140(2)$ & $33(1)$ \\
\hline$C(12)$ & $-4018(6)$ & $2628(4)$ & $7562(2)$ & $39(1)$ \\
\hline$C(13)$ & $-4902(7)$ & 2551(4) & $7932(2)$ & $48(2)$ \\
\hline$C(14)$ & $-6067(7)$ & $2037(5)$ & 7883(3) & $57(2)$ \\
\hline$C(15)$ & $-6355(8)$ & $1603(5)$ & 7471(3) & $61(2)$ \\
\hline$C(16)$ & $-5496(7)$ & $1676(4)$ & 7094(2) & $48(2)$ \\
\hline $\mathrm{C}(21)$ & $-2710(6)$ & $3521(4)$ & $6677(2)$ & $30(1)$ \\
\hline $\mathrm{C}(22)$ & $-3728(7)$ & 4149(4) & $6572(2)$ & $43(1)$ \\
\hline$C(23)$ & $-3440(8)$ & $5079(4)$ & $6580(2)$ & $53(2)$ \\
\hline $\mathrm{C}(24)$ & $-2138(8)$ & $5354(4)$ & $6714(2)$ & $53(2)$ \\
\hline $\mathrm{C}(25)$ & $-1170(7)$ & $4740(5)$ & 6830(3) & $55(2)$ \\
\hline$C(26)$ & $-1445(7)$ & $3802(4)$ & 6812(2) & $48(2)$ \\
\hline$C(31)$ & $-3990(6)$ & 1989(4) & $6120(2)$ & $33(1)$ \\
\hline$C(32)$ & $-4255(7)$ & $1053(5)$ & $6060(2)$ & $49(2)$ \\
\hline$C(33)$ & $-4830(7)$ & $756(5)$ & $5637(3)$ & $59(2)$ \\
\hline$C(34)$ & $-5108(8)$ & 1371(5) & $5286(3)$ & $59(2)$ \\
\hline$C(35)$ & $-4873(8)$ & $2264(5)$ & $5342(2)$ & $62(2)$ \\
\hline$C(36)$ & $-4291(7)$ & 2591(5) & $5765(2)$ & $49(2)$ \\
\hline $\mathrm{C}(41)$ & $-1734(6)$ & $1565(3)$ & $6766(2)$ & $30(1)$ \\
\hline$C(42)$ & $-666(6)$ & $1523(4)$ & $6455(2)$ & $39(1)$ \\
\hline$C(43)$ & $367(7)$ & $893(4)$ & $6556(2)$ & $45(2)$ \\
\hline$C(44)$ & $294(7)$ & $298(4)$ & $6927(2)$ & $46(2)$ \\
\hline
\end{tabular}




\begin{tabular}{lrrrr}
$\mathrm{C}(45)$ & $-788(7)$ & $309(4)$ & $7208(2)$ & $45(2)$ \\
$\mathrm{C}(46)$ & $-1824(6)$ & $939(4)$ & $7136(2)$ & $38(1)$ \\
$\mathrm{C}(51)$ & $-1187(7)$ & $1361(5)$ & $5468(2)$ & $46(2)$ \\
$\mathrm{C}(52)$ & $-1212(8)$ & $433(5)$ & $5543(3)$ & $61(2)$ \\
$\mathrm{C}(53)$ & $-1590(9)$ & $-176(6)$ & $5191(4)$ & $82(3)$ \\
$\mathrm{C}(54)$ & $-1954(9)$ & $172(8)$ & $4749(3)$ & $84(3)$ \\
$\mathrm{C}(55)$ & $-1938(9)$ & $1082(7)$ & $4669(3)$ & $76(2)$ \\
$\mathrm{C}(56)$ & $-1591(8)$ & $1699(6)$ & $5027(3)$ & $65(2)$ \\
$\mathrm{C}(61)$ & $1885(7)$ & $3364(5)$ & $6162(3)$ & $52(2)$ \\
$\mathrm{C}(62)$ & $2281(8)$ & $4219(5)$ & $6011(3)$ & $59(2)$ \\
$\mathrm{C}(63)$ & $2738(8)$ & $4866(5)$ & $6316(3)$ & $63(2)$ \\
$\mathrm{C}(64)$ & $2845(7)$ & $4711(5)$ & $6791(3)$ & $60(2)$ \\
$\mathrm{C}(65)$ & $2459(7)$ & $3871(6)$ & $6962(3)$ & $61(2)$ \\
$\mathrm{C}(66)$ & $1973(7)$ & $3204(5)$ & $6657(2)$ & $53(2)$ \\
$\mathrm{C}(71)$ & $1098(8)$ & $2995(5)$ & $5199(2)$ & $53(2)$ \\
$\mathrm{C}(72)$ & $136(9)$ & $3688(6)$ & $5142(3)$ & $69(2)$ \\
$\mathrm{C}(73)$ & $-209(10)$ & $4014(7)$ & $4683(3)$ & $86(3)$ \\
$\mathrm{C}(74)$ & $424(12)$ & $3688(7)$ & $4301(3)$ & $93(3)$ \\
$\mathrm{C}(75)$ & $1422(12)$ & $3064(7)$ & $4365(3)$ & $94(3)$ \\
$\mathrm{C}(76)$ & $1771(8)$ & $2723(6)$ & $4806(2)$ & $63(2)$ \\
& & & & \\
\hline
\end{tabular}


Table S16. Bond lengths $[\AA]$ and angles $\left[{ }^{\circ}\right]$ for dal0303 1-diphosphino-2-phosphoniumbenze $\left(3 \mathrm{GaCl} 4 \bullet 0.5 \mathrm{CH}_{2} \mathrm{Cl}_{2}\right)$.

\begin{tabular}{|c|c|}
\hline $\mathrm{Ga}-\mathrm{Cl}(4)$ & $2.151(2)$ \\
\hline $\mathrm{Ga}-\mathrm{Cl}(1)$ & $2.159(2)$ \\
\hline $\mathrm{Ga}-\mathrm{Cl}(3)$ & $2.161(2)$ \\
\hline $\mathrm{Ga}-\mathrm{Cl}(2)$ & $2.164(2)$ \\
\hline $\mathrm{P}(1)-\mathrm{C}(31)$ & $1.803(6)$ \\
\hline $\mathrm{P}(1)-\mathrm{C}(21)$ & $1.805(5)$ \\
\hline $\mathrm{P}(1)-\mathrm{C}(11)$ & $1.810(6)$ \\
\hline $\mathrm{P}(1)-\mathrm{C}(41)$ & $1.813(5)$ \\
\hline $\mathrm{P}(2)-\mathrm{C}(42)$ & $1.840(6)$ \\
\hline $\mathrm{P}(2)-\mathrm{C}(51)$ & $1.855(7)$ \\
\hline $\mathrm{P}(2)-\mathrm{P}(3)$ & $2.243(2)$ \\
\hline $\mathrm{P}(3)-\mathrm{C}(71)$ & $1.832(7)$ \\
\hline $\mathrm{P}(3)-\mathrm{C}(61)$ & $1.848(7)$ \\
\hline$C(11)-C(16)$ & $1.373(8)$ \\
\hline$C(11)-C(12)$ & $1.393(8)$ \\
\hline$C(12)-C(13)$ & $1.390(8)$ \\
\hline$C(13)-C(14)$ & $1.378(10)$ \\
\hline$C(14)-C(15)$ & $1.359(11)$ \\
\hline$C(15)-C(16)$ & $1.389(9)$ \\
\hline$C(21)-C(26)$ & $1.362(8)$ \\
\hline$C(21)-C(22)$ & $1.388(8)$ \\
\hline$C(22)-C(23)$ & $1.387(9)$ \\
\hline$C(23)-C(24)$ & $1.391(11)$ \\
\hline$C(24)-C(25)$ & $1.347(10)$ \\
\hline$C(25)-C(26)$ & $1.397(9)$ \\
\hline$C(31)-C(36)$ & $1.367(8)$ \\
\hline $\mathrm{C}(31)-\mathrm{C}(32)$ & $1.401(8)$ \\
\hline$C(32)-C(33)$ & $1.388(9)$ \\
\hline $\mathrm{C}(33)-\mathrm{C}(34)$ & $1.366(10)$ \\
\hline$C(34)-C(35)$ & $1.333(10)$ \\
\hline$C(35)-C(36)$ & $1.403(9)$ \\
\hline $\mathrm{C}(41)-\mathrm{C}(42)$ & $1.396(8)$ \\
\hline C(41)-C(46) & $1.400(7)$ \\
\hline
\end{tabular}




\begin{tabular}{|c|c|}
\hline$C(42)-C(43)$ & $1.399(8)$ \\
\hline$C(43)-C(44)$ & $1.371(8)$ \\
\hline$C(44)-C(45)$ & $1.352(9)$ \\
\hline$C(45)-C(46)$ & $1.387(9)$ \\
\hline$C(51)-C(52)$ & $1.373(9)$ \\
\hline$C(51)-C(56)$ & $1.399(10)$ \\
\hline$C(52)-C(53)$ & $1.384(11)$ \\
\hline$C(53)-C(54)$ & $1.395(13)$ \\
\hline$C(54)-C(55)$ & $1.349(13)$ \\
\hline$C(55)-C(56)$ & $1.396(11)$ \\
\hline$C(61)-C(62)$ & $1.380(9)$ \\
\hline$C(61)-C(66)$ & $1.428(10)$ \\
\hline$C(62)-C(63)$ & $1.354(10)$ \\
\hline$C(63)-C(64)$ & $1.372(11)$ \\
\hline$C(64)-C(65)$ & $1.375(11)$ \\
\hline$C(65)-C(66)$ & $1.383(10)$ \\
\hline$C(71)-C(76)$ & $1.375(9)$ \\
\hline $\mathrm{C}(71)-\mathrm{C}(72)$ & $1.395(10)$ \\
\hline$C(72)-C(73)$ & $1.425(11)$ \\
\hline$C(73)-C(74)$ & $1.353(12)$ \\
\hline$C(74)-C(75)$ & $1.352(13)$ \\
\hline$C(75)-C(76)$ & $1.386(11)$ \\
\hline $\mathrm{Cl}(4)-\mathrm{Ga}-\mathrm{Cl}(1)$ & $110.69(9)$ \\
\hline $\mathrm{Cl}(4)-\mathrm{Ga}-\mathrm{Cl}(3)$ & $111.00(11)$ \\
\hline $\mathrm{Cl}(1)-\mathrm{Ga}-\mathrm{Cl}(3)$ & $106.28(10)$ \\
\hline $\mathrm{Cl}(4)-\mathrm{Ga}-\mathrm{Cl}(2)$ & $109.44(9)$ \\
\hline $\mathrm{Cl}(1)-\mathrm{Ga}-\mathrm{Cl}(2)$ & $110.01(11)$ \\
\hline $\mathrm{Cl}(3)-\mathrm{Ga}-\mathrm{Cl}(2)$ & $109.37(14)$ \\
\hline $\mathrm{C}(31)-\mathrm{P}(1)-\mathrm{C}(21)$ & 112.7(3) \\
\hline $\mathrm{C}(31)-\mathrm{P}(1)-\mathrm{C}(11)$ & 109.2(3) \\
\hline $\mathrm{C}(21)-\mathrm{P}(1)-\mathrm{C}(11)$ & $104.9(2)$ \\
\hline $\mathrm{C}(31)-\mathrm{P}(1)-\mathrm{C}(41)$ & $107.6(2)$ \\
\hline $\mathrm{C}(21)-\mathrm{P}(1)-\mathrm{C}(41)$ & $113.4(3)$ \\
\hline $\mathrm{C}(11)-\mathrm{P}(1)-\mathrm{C}(41)$ & $108.9(2)$ \\
\hline $\mathrm{C}(42)-\mathrm{P}(2)-\mathrm{C}(51)$ & $101.1(3)$ \\
\hline
\end{tabular}




\begin{tabular}{|c|c|}
\hline $\mathrm{C}(42)-\mathrm{P}(2)-\mathrm{P}(3)$ & $102.1(2)$ \\
\hline $\mathrm{C}(51)-\mathrm{P}(2)-\mathrm{P}(3)$ & $98.3(2)$ \\
\hline $\mathrm{C}(71)-\mathrm{P}(3)-\mathrm{C}(61)$ & $103.0(3)$ \\
\hline $\mathrm{C}(71)-\mathrm{P}(3)-\mathrm{P}(2)$ & $94.3(2)$ \\
\hline C(61)-P(3)-P(2) & $100.1(2)$ \\
\hline$C(16)-C(11)-C(12)$ & $120.1(5)$ \\
\hline $\mathrm{C}(16)-\mathrm{C}(11)-\mathrm{P}(1)$ & $122.6(5)$ \\
\hline $\mathrm{C}(12)-\mathrm{C}(11)-\mathrm{P}(1)$ & $117.3(4)$ \\
\hline $\mathrm{C}(13)-\mathrm{C}(12)-\mathrm{C}(11)$ & $119.0(6)$ \\
\hline$C(14)-C(13)-C(12)$ & $120.6(6)$ \\
\hline$C(15)-C(14)-C(13)$ & $119.7(6)$ \\
\hline$C(14)-C(15)-C(16)$ & $120.9(7)$ \\
\hline $\mathrm{C}(11)-\mathrm{C}(16)-\mathrm{C}(15)$ & $119.7(6)$ \\
\hline $\mathrm{C}(26)-\mathrm{C}(21)-\mathrm{C}(22)$ & $121.1(5)$ \\
\hline $\mathrm{C}(26)-\mathrm{C}(21)-\mathrm{P}(1)$ & $121.5(5)$ \\
\hline $\mathrm{C}(22)-\mathrm{C}(21)-\mathrm{P}(1)$ & $117.2(5)$ \\
\hline$C(23)-C(22)-C(21)$ & $119.7(6)$ \\
\hline$C(22)-C(23)-C(24)$ & $118.3(6)$ \\
\hline$C(25)-C(24)-C(23)$ & $121.4(6)$ \\
\hline$C(24)-C(25)-C(26)$ & $120.6(7)$ \\
\hline$C(21)-C(26)-C(25)$ & $118.8(6)$ \\
\hline $\mathrm{C}(36)-\mathrm{C}(31)-\mathrm{C}(32)$ & $120.1(6)$ \\
\hline $\mathrm{C}(36)-\mathrm{C}(31)-\mathrm{P}(1)$ & $123.2(4)$ \\
\hline C(32)-C(31)-P(1) & $116.6(4)$ \\
\hline $\mathrm{C}(33)-\mathrm{C}(32)-\mathrm{C}(31)$ & $118.8(6)$ \\
\hline $\mathrm{C}(34)-\mathrm{C}(33)-\mathrm{C}(32)$ & $119.9(7)$ \\
\hline $\mathrm{C}(35)-\mathrm{C}(34)-\mathrm{C}(33)$ & $121.7(7)$ \\
\hline $\mathrm{C}(34)-\mathrm{C}(35)-\mathrm{C}(36)$ & $120.1(7)$ \\
\hline $\mathrm{C}(31)-\mathrm{C}(36)-\mathrm{C}(35)$ & $119.4(6)$ \\
\hline $\mathrm{C}(42)-\mathrm{C}(41)-\mathrm{C}(46)$ & $120.8(5)$ \\
\hline C(42)-C(41)-P(1) & $121.8(4)$ \\
\hline $\mathrm{C}(46)-\mathrm{C}(41)-\mathrm{P}(1)$ & $117.0(4)$ \\
\hline$C(41)-C(42)-C(43)$ & $117.4(5)$ \\
\hline C(41)-C(42)-P(2) & 118.6(4) \\
\hline $\mathrm{C}(43)-\mathrm{C}(42)-\mathrm{P}(2)$ & $123.9(5)$ \\
\hline$C(44)-C(43)-C(42)$ & $121.4(6)$ \\
\hline
\end{tabular}




$\begin{array}{ll}\mathrm{C}(45)-\mathrm{C}(44)-\mathrm{C}(43) & 120.5(6) \\ \mathrm{C}(44)-\mathrm{C}(45)-\mathrm{C}(46) & 120.8(5) \\ \mathrm{C}(45)-\mathrm{C}(46)-\mathrm{C}(41) & 118.9(5) \\ \mathrm{C}(52)-\mathrm{C}(51)-\mathrm{C}(56) & 118.9(7) \\ \mathrm{C}(52)-\mathrm{C}(51)-\mathrm{P}(2) & 125.1(5) \\ \mathrm{C}(56)-\mathrm{C}(51)-\mathrm{P}(2) & 116.0(5) \\ \mathrm{C}(51)-\mathrm{C}(52)-\mathrm{C}(53) & 121.8(8) \\ \mathrm{C}(52)-\mathrm{C}(53)-\mathrm{C}(54) & 118.6(8) \\ \mathrm{C}(55)-\mathrm{C}(54)-\mathrm{C}(53) & 120.5(8) \\ \mathrm{C}(54)-\mathrm{C}(55)-\mathrm{C}(56) & 121.1(9) \\ \mathrm{C}(55)-\mathrm{C}(56)-\mathrm{C}(51) & 119.1(8) \\ \mathrm{C}(62)-\mathrm{C}(61)-\mathrm{C}(66) & 116.5(7) \\ \mathrm{C}(62)-\mathrm{C}(61)-\mathrm{P}(3) & 123.5(6) \\ \mathrm{C}(66)-\mathrm{C}(61)-\mathrm{P}(3) & 119.7(5) \\ \mathrm{C}(63)-\mathrm{C}(62)-\mathrm{C}(61) & 121.5(7) \\ \mathrm{C}(62)-\mathrm{C}(63)-\mathrm{C}(64) & 122.3(7) \\ \mathrm{C}(63)-\mathrm{C}(64)-\mathrm{C}(65) & 118.6(7) \\ \mathrm{C}(64)-\mathrm{C}(65)-\mathrm{C}(66) & 120.1(7) \\ \mathrm{C}(65)-\mathrm{C}(66)-\mathrm{C}(61) & 120.9(7) \\ \mathrm{C}(76)-\mathrm{C}(71)-\mathrm{C}(72) & 117.2(7) \\ \mathrm{C}(76)-\mathrm{C}(71)-\mathrm{P}(3) & 119.4(5) \\ \mathrm{C}(72)-\mathrm{C}(71)-\mathrm{P}(3) & 123.3(5) \\ \mathrm{C}(71)-\mathrm{C}(72)-\mathrm{C}(73) & 119.7(7) \\ \mathrm{C}(74)-\mathrm{C}(73)-\mathrm{C}(72) & 121.0(8) \\ \mathrm{C}(75)-\mathrm{C}(74)-\mathrm{C}(73) & 118.7(8) \\ \mathrm{C}(74)-\mathrm{C}(75)-\mathrm{C}(76) & 121.8(8) \\ \mathrm{C}(71)-\mathrm{C}(76)-\mathrm{C}(75) & 121.3(8) \\ & \end{array}$

Symmetry transformations used to generate equivalent atoms: 
Table S17. Anisotropic displacement parameters $\left(\AA^{2} \times 10^{3}\right)$ for dal0303 1-diphosphino-2-

phosphoniumbenze (3GaCl4•0.5 $\left.\mathbf{C H}_{\mathbf{2}} \mathbf{C l}_{\mathbf{2}}\right)$. The anisotropic displacement factor exponent takes the form: $2 \pi^{2}\left[\mathrm{~h}^{2} \mathrm{a}^{* 2} \mathrm{U}^{11}+\ldots+2 \mathrm{hk} \mathrm{a} \mathrm{b}^{*} \mathrm{U}^{12}\right]$

\begin{tabular}{|c|c|c|c|c|c|c|}
\hline & $\mathrm{U}^{11}$ & $\mathrm{U}^{22}$ & $\mathrm{U}^{33}$ & $\mathrm{U}^{23}$ & $\mathrm{U}^{13}$ & $\mathrm{U}^{12}$ \\
\hline $\mathrm{Ga}$ & $46(1)$ & $40(1)$ & $43(1)$ & $-4(1)$ & $3(1)$ & $-6(1)$ \\
\hline $\mathrm{Cl}(1)$ & $81(2)$ & $92(2)$ & $61(1)$ & $-31(1)$ & $-5(1)$ & $-12(1)$ \\
\hline $\mathrm{Cl}(2)$ & $54(1)$ & $77(2)$ & 194(3) & $-38(2)$ & $26(2)$ & $-5(1)$ \\
\hline $\mathrm{Cl}(3)$ & 192(3) & $83(2)$ & $62(1)$ & $22(1)$ & $-19(2)$ & $-20(2)$ \\
\hline $\mathrm{Cl}(4)$ & $65(1)$ & $62(1)$ & $90(1)$ & $-1(1)$ & $13(1)$ & $-24(1)$ \\
\hline $\mathrm{P}(1)$ & $28(1)$ & $27(1)$ & $30(1)$ & $1(1)$ & $0(1)$ & $0(1)$ \\
\hline $\mathrm{P}(2)$ & $40(1)$ & $48(1)$ & $36(1)$ & $5(1)$ & $5(1)$ & $6(1)$ \\
\hline $\mathrm{P}(3)$ & $40(1)$ & $54(1)$ & $47(1)$ & $9(1)$ & $9(1)$ & $7(1)$ \\
\hline $\mathrm{C}(11)$ & $32(3)$ & $30(3)$ & $37(3)$ & $6(2)$ & $3(2)$ & $6(2)$ \\
\hline$C(12)$ & $39(3)$ & $41(3)$ & $38(3)$ & $-1(3)$ & $7(3)$ & $0(3)$ \\
\hline$C(13)$ & $58(4)$ & $46(4)$ & $42(3)$ & $7(3)$ & $15(3)$ & $8(3)$ \\
\hline$C(14)$ & $41(4)$ & $75(5)$ & $58(4)$ & $18(4)$ & $18(3)$ & $10(4)$ \\
\hline$C(15)$ & $54(5)$ & $72(5)$ & $57(4)$ & $24(4)$ & $6(4)$ & $-16(4)$ \\
\hline$C(16)$ & $38(4)$ & $59(4)$ & $46(4)$ & $5(3)$ & $0(3)$ & $-15(3)$ \\
\hline $\mathrm{C}(21)$ & $38(3)$ & $28(3)$ & $23(3)$ & $1(2)$ & $5(2)$ & $-3(2)$ \\
\hline$C(22)$ & $43(4)$ & $40(3)$ & $45(3)$ & $-4(3)$ & $-1(3)$ & $4(3)$ \\
\hline$C(23)$ & $72(5)$ & $31(3)$ & $56(4)$ & $0(3)$ & $9(4)$ & $10(3)$ \\
\hline$C(24)$ & $80(6)$ & $33(3)$ & $49(4)$ & $-4(3)$ & $28(4)$ & $-10(3)$ \\
\hline$C(25)$ & $42(4)$ & $52(4)$ & $71(5)$ & $-8(4)$ & $5(4)$ & $-21(3)$ \\
\hline$C(26)$ & $37(4)$ & $37(3)$ & $68(4)$ & $-5(3)$ & $-5(3)$ & $-3(3)$ \\
\hline $\mathrm{C}(31)$ & $29(3)$ & $35(3)$ & $34(3)$ & $-7(2)$ & $-5(2)$ & 1(2) \\
\hline$C(32)$ & $49(4)$ & $49(4)$ & $50(4)$ & $-5(3)$ & $-3(3)$ & $-1(3)$ \\
\hline$C(33)$ & $47(4)$ & $54(4)$ & $75(5)$ & $-22(4)$ & $-14(4)$ & $-5(3)$ \\
\hline$C(34)$ & $50(4)$ & $74(5)$ & $52(4)$ & $-21(4)$ & $-16(3)$ & $8(4)$ \\
\hline$C(35)$ & $78(6)$ & $64(5)$ & $45(4)$ & $3(3)$ & $-15(4)$ & 6(4) \\
\hline$C(36)$ & $49(4)$ & $52(4)$ & $44(3)$ & $-1(3)$ & $-19(3)$ & $3(3)$ \\
\hline$C(41)$ & $33(3)$ & $23(2)$ & $32(3)$ & $1(2)$ & $-4(2)$ & $4(2)$ \\
\hline$C(42)$ & $36(3)$ & $41(3)$ & $38(3)$ & $10(3)$ & $0(3)$ & $6(3)$ \\
\hline$C(43)$ & $43(4)$ & $49(4)$ & $43(3)$ & $7(3)$ & $15(3)$ & $12(3)$ \\
\hline $\mathrm{C}(44)$ & 45(4) & $42(3)$ & $51(4)$ & $4(3)$ & $-1(3)$ & $16(3)$ \\
\hline
\end{tabular}




\begin{tabular}{lllllll}
$\mathrm{C}(45)$ & $50(4)$ & $39(3)$ & $46(3)$ & $14(3)$ & $5(3)$ & $6(3)$ \\
$\mathrm{C}(46)$ & $41(4)$ & $36(3)$ & $36(3)$ & $5(2)$ & $7(3)$ & $4(3)$ \\
$\mathrm{C}(51)$ & $41(4)$ & $57(4)$ & $41(3)$ & $-1(3)$ & $4(3)$ & $5(3)$ \\
$\mathrm{C}(52)$ & $66(5)$ & $51(4)$ & $66(5)$ & $-8(4)$ & $-1(4)$ & $-5(4)$ \\
$\mathrm{C}(53)$ & $80(6)$ & $62(5)$ & $103(7)$ & $-29(5)$ & $-7(6)$ & $2(4)$ \\
$\mathrm{C}(54)$ & $56(5)$ & $123(8)$ & $72(6)$ & $-50(6)$ & $-4(5)$ & $-5(5)$ \\
$\mathrm{C}(55)$ & $61(5)$ & $113(8)$ & $53(5)$ & $-22(5)$ & $-12(4)$ & $-4(5)$ \\
$\mathrm{C}(56)$ & $50(5)$ & $88(6)$ & $57(4)$ & $-3(4)$ & $-6(4)$ & $3(4)$ \\
$\mathrm{C}(61)$ & $38(4)$ & $57(4)$ & $61(4)$ & $0(3)$ & $-5(3)$ & $5(3)$ \\
$\mathrm{C}(62)$ & $70(5)$ & $49(4)$ & $58(4)$ & $11(3)$ & $-16(4)$ & $-2(4)$ \\
$\mathrm{C}(63)$ & $59(5)$ & $50(4)$ & $79(5)$ & $6(4)$ & $-11(4)$ & $-1(4)$ \\
$\mathrm{C}(64)$ & $39(4)$ & $59(5)$ & $83(6)$ & $-20(4)$ & $-8(4)$ & $0(3)$ \\
$\mathrm{C}(65)$ & $46(4)$ & $86(6)$ & $52(4)$ & $-8(4)$ & $0(3)$ & $4(4)$ \\
$\mathrm{C}(66)$ & $40(4)$ & $58(4)$ & $61(4)$ & $14(3)$ & $-6(3)$ & $-7(3)$ \\
$\mathrm{C}(71)$ & $59(5)$ & $55(4)$ & $45(4)$ & $5(3)$ & $18(3)$ & $9(3)$ \\
$\mathrm{C}(72)$ & $77(6)$ & $74(5)$ & $55(4)$ & $22(4)$ & $13(4)$ & $11(4)$ \\
$\mathrm{C}(73)$ & $90(7)$ & $92(6)$ & $77(6)$ & $36(5)$ & $4(5)$ & $22(5)$ \\
$\mathrm{C}(74)$ & $120(9)$ & $109(8)$ & $52(5)$ & $25(5)$ & $25(6)$ & $23(7)$ \\
$\mathrm{C}(75)$ & $116(9)$ & $121(8)$ & $45(5)$ & $10(5)$ & $23(5)$ & $23(7)$ \\
$\mathrm{C}(76)$ & $52(5)$ & $81(5)$ & $56(4)$ & $8(4)$ & $17(4)$ & $5(4)$ \\
& & & & & & \\
\hline
\end{tabular}


Table S19. Torsion angles $\left[{ }^{\circ}\right]$ for dal0303 1-diphosphino-2-phosphoniumbenze $\left(\mathbf{3 G a C l 4} \cdot \mathbf{0 . 5} \mathbf{C H}_{\mathbf{2}} \mathbf{C l}_{\mathbf{2}}\right)$.

\begin{tabular}{|c|c|}
\hline $\mathrm{C}(42)-\mathrm{P}(2)-\mathrm{P}(3)-\mathrm{C}(71)$ & $173.7(3)$ \\
\hline $\mathrm{C}(51)-\mathrm{P}(2)-\mathrm{P}(3)-\mathrm{C}(71)$ & $70.4(3)$ \\
\hline $\mathrm{C}(42)-\mathrm{P}(2)-\mathrm{P}(3)-\mathrm{C}(61)$ & $-82.3(3)$ \\
\hline $\mathrm{C}(51)-\mathrm{P}(2)-\mathrm{P}(3)-\mathrm{C}(61)$ & $174.4(3)$ \\
\hline $\mathrm{C}(31)-\mathrm{P}(1)-\mathrm{C}(11)-\mathrm{C}(16)$ & $16.0(6)$ \\
\hline $\mathrm{C}(21)-\mathrm{P}(1)-\mathrm{C}(11)-\mathrm{C}(16)$ & $137.0(5)$ \\
\hline $\mathrm{C}(41)-\mathrm{P}(1)-\mathrm{C}(11)-\mathrm{C}(16)$ & $-101.3(5)$ \\
\hline $\mathrm{C}(31)-\mathrm{P}(1)-\mathrm{C}(11)-\mathrm{C}(12)$ & $-163.5(4)$ \\
\hline $\mathrm{C}(21)-\mathrm{P}(1)-\mathrm{C}(11)-\mathrm{C}(12)$ & $-42.5(5)$ \\
\hline $\mathrm{C}(41)-\mathrm{P}(1)-\mathrm{C}(11)-\mathrm{C}(12)$ & $79.2(5)$ \\
\hline $\mathrm{C}(16)-\mathrm{C}(11)-\mathrm{C}(12)-\mathrm{C}(13)$ & $-0.6(9)$ \\
\hline $\mathrm{P}(1)-\mathrm{C}(11)-\mathrm{C}(12)-\mathrm{C}(13)$ & $178.9(4)$ \\
\hline $\mathrm{C}(11)-\mathrm{C}(12)-\mathrm{C}(13)-\mathrm{C}(14)$ & $0.7(9)$ \\
\hline$C(12)-C(13)-C(14)-C(15)$ & $-0.2(10)$ \\
\hline$C(13)-C(14)-C(15)-C(16)$ & $-0.5(11)$ \\
\hline$C(12)-C(11)-C(16)-C(15)$ & $-0.1(10)$ \\
\hline $\mathrm{P}(1)-\mathrm{C}(11)-\mathrm{C}(16)-\mathrm{C}(15)$ & $-179.5(5)$ \\
\hline$C(14)-C(15)-C(16)-C(11)$ & $0.6(11)$ \\
\hline $\mathrm{C}(31)-\mathrm{P}(1)-\mathrm{C}(21)-\mathrm{C}(26)$ & $-130.9(5)$ \\
\hline $\mathrm{C}(11)-\mathrm{P}(1)-\mathrm{C}(21)-\mathrm{C}(26)$ & $110.4(5)$ \\
\hline $\mathrm{C}(41)-\mathrm{P}(1)-\mathrm{C}(21)-\mathrm{C}(26)$ & $-8.3(6)$ \\
\hline $\mathrm{C}(31)-\mathrm{P}(1)-\mathrm{C}(21)-\mathrm{C}(22)$ & $53.2(5)$ \\
\hline $\mathrm{C}(11)-\mathrm{P}(1)-\mathrm{C}(21)-\mathrm{C}(22)$ & $-65.5(5)$ \\
\hline $\mathrm{C}(41)-\mathrm{P}(1)-\mathrm{C}(21)-\mathrm{C}(22)$ & $175.7(4)$ \\
\hline$C(26)-C(21)-C(22)-C(23)$ & $3.4(9)$ \\
\hline $\mathrm{P}(1)-\mathrm{C}(21)-\mathrm{C}(22)-\mathrm{C}(23)$ & $179.4(5)$ \\
\hline $\mathrm{C}(21)-\mathrm{C}(22)-\mathrm{C}(23)-\mathrm{C}(24)$ & $-2.7(9)$ \\
\hline $\mathrm{C}(22)-\mathrm{C}(23)-\mathrm{C}(24)-\mathrm{C}(25)$ & $0.6(10)$ \\
\hline$C(23)-C(24)-C(25)-C(26)$ & $0.9(11)$ \\
\hline $\mathrm{C}(22)-\mathrm{C}(21)-\mathrm{C}(26)-\mathrm{C}(25)$ & $-2.0(9)$ \\
\hline $\mathrm{P}(1)-\mathrm{C}(21)-\mathrm{C}(26)-\mathrm{C}(25)$ & $-177.8(5)$ \\
\hline $\mathrm{C}(24)-\mathrm{C}(25)-\mathrm{C}(26)-\mathrm{C}(21)$ & $-0.2(10)$ \\
\hline C(21)-P(1)-C(31)-C(36) & $-2.9(6)$ \\
\hline $\mathrm{C}(11)-\mathrm{P}(1)-\mathrm{C}(31)-\mathrm{C}(36)$ & $113.3(5)$ \\
\hline
\end{tabular}




\begin{tabular}{|c|c|}
\hline $\mathrm{C}(41)-\mathrm{P}(1)-\mathrm{C}(31)-\mathrm{C}(36)$ & $-128.6(5)$ \\
\hline $\mathrm{C}(21)-\mathrm{P}(1)-\mathrm{C}(31)-\mathrm{C}(32)$ & $173.7(5)$ \\
\hline $\mathrm{C}(11)-\mathrm{P}(1)-\mathrm{C}(31)-\mathrm{C}(32)$ & $-70.1(5)$ \\
\hline $\mathrm{C}(41)-\mathrm{P}(1)-\mathrm{C}(31)-\mathrm{C}(32)$ & $48.0(5)$ \\
\hline $\mathrm{C}(36)-\mathrm{C}(31)-\mathrm{C}(32)-\mathrm{C}(33)$ & $-0.3(10)$ \\
\hline$P(1)-C(31)-C(32)-C(33)$ & $-177.0(5)$ \\
\hline $\mathrm{C}(31)-\mathrm{C}(32)-\mathrm{C}(33)-\mathrm{C}(34)$ & $0.9(10)$ \\
\hline$C(32)-C(33)-C(34)-C(35)$ & $-1.7(12)$ \\
\hline $\mathrm{C}(33)-\mathrm{C}(34)-\mathrm{C}(35)-\mathrm{C}(36)$ & $1.8(12)$ \\
\hline $\mathrm{C}(32)-\mathrm{C}(31)-\mathrm{C}(36)-\mathrm{C}(35)$ & $0.3(10)$ \\
\hline $\mathrm{P}(1)-\mathrm{C}(31)-\mathrm{C}(36)-\mathrm{C}(35)$ & $176.8(5)$ \\
\hline $\mathrm{C}(34)-\mathrm{C}(35)-\mathrm{C}(36)-\mathrm{C}(31)$ & $-1.1(11)$ \\
\hline $\mathrm{C}(31)-\mathrm{P}(1)-\mathrm{C}(41)-\mathrm{C}(42)$ & $61.6(5)$ \\
\hline $\mathrm{C}(21)-\mathrm{P}(1)-\mathrm{C}(41)-\mathrm{C}(42)$ & $-63.7(5)$ \\
\hline $\mathrm{C}(11)-\mathrm{P}(1)-\mathrm{C}(41)-\mathrm{C}(42)$ & $179.9(5)$ \\
\hline $\mathrm{C}(31)-\mathrm{P}(1)-\mathrm{C}(41)-\mathrm{C}(46)$ & $-110.5(5)$ \\
\hline $\mathrm{C}(21)-\mathrm{P}(1)-\mathrm{C}(41)-\mathrm{C}(46)$ & $124.2(4)$ \\
\hline $\mathrm{C}(11)-\mathrm{P}(1)-\mathrm{C}(41)-\mathrm{C}(46)$ & $7.8(5)$ \\
\hline $\mathrm{C}(46)-\mathrm{C}(41)-\mathrm{C}(42)-\mathrm{C}(43)$ & $-6.0(9)$ \\
\hline $\mathrm{P}(1)-\mathrm{C}(41)-\mathrm{C}(42)-\mathrm{C}(43)$ & $-177.9(5)$ \\
\hline $\mathrm{C}(46)-\mathrm{C}(41)-\mathrm{C}(42)-\mathrm{P}(2)$ & $169.9(5)$ \\
\hline $\mathrm{P}(1)-\mathrm{C}(41)-\mathrm{C}(42)-\mathrm{P}(2)$ & $-2.0(7)$ \\
\hline $\mathrm{C}(51)-\mathrm{P}(2)-\mathrm{C}(42)-\mathrm{C}(41)$ & $-104.4(5)$ \\
\hline $\mathrm{P}(3)-\mathrm{P}(2)-\mathrm{C}(42)-\mathrm{C}(41)$ & $154.5(4)$ \\
\hline $\mathrm{C}(51)-\mathrm{P}(2)-\mathrm{C}(42)-\mathrm{C}(43)$ & $71.2(6)$ \\
\hline $\mathrm{P}(3)-\mathrm{P}(2)-\mathrm{C}(42)-\mathrm{C}(43)$ & $-29.9(6)$ \\
\hline $\mathrm{C}(41)-\mathrm{C}(42)-\mathrm{C}(43)-\mathrm{C}(44)$ & $4.2(10)$ \\
\hline $\mathrm{P}(2)-\mathrm{C}(42)-\mathrm{C}(43)-\mathrm{C}(44)$ & $-171.5(5)$ \\
\hline $\mathrm{C}(42)-\mathrm{C}(43)-\mathrm{C}(44)-\mathrm{C}(45)$ & $-0.2(10)$ \\
\hline $\mathrm{C}(43)-\mathrm{C}(44)-\mathrm{C}(45)-\mathrm{C}(46)$ & $-2.1(10)$ \\
\hline $\mathrm{C}(44)-\mathrm{C}(45)-\mathrm{C}(46)-\mathrm{C}(41)$ & $0.2(10)$ \\
\hline $\mathrm{C}(42)-\mathrm{C}(41)-\mathrm{C}(46)-\mathrm{C}(45)$ & $4.0(9)$ \\
\hline $\mathrm{P}(1)-\mathrm{C}(41)-\mathrm{C}(46)-\mathrm{C}(45)$ & $176.2(5)$ \\
\hline $\mathrm{C}(42)-\mathrm{P}(2)-\mathrm{C}(51)-\mathrm{C}(52)$ & $-8.1(7)$ \\
\hline $\mathrm{P}(3)-\mathrm{P}(2)-\mathrm{C}(51)-\mathrm{C}(52)$ & $96.0(6)$ \\
\hline $\mathrm{C}(42)-\mathrm{P}(2)-\mathrm{C}(51)-\mathrm{C}(56)$ & $168.0(5)$ \\
\hline
\end{tabular}




\begin{tabular}{|c|c|}
\hline $\mathrm{P}(3)-\mathrm{P}(2)-\mathrm{C}(51)-\mathrm{C}(56)$ & $-87.8(5)$ \\
\hline$C(56)-C(51)-C(52)-C(53)$ & $1.7(11)$ \\
\hline $\mathrm{P}(2)-\mathrm{C}(51)-\mathrm{C}(52)-\mathrm{C}(53)$ & $177.8(6)$ \\
\hline$C(51)-C(52)-C(53)-C(54)$ & $0.0(13)$ \\
\hline $\mathrm{C}(52)-\mathrm{C}(53)-\mathrm{C}(54)-\mathrm{C}(55)$ & $-0.2(14)$ \\
\hline $\mathrm{C}(53)-\mathrm{C}(54)-\mathrm{C}(55)-\mathrm{C}(56)$ & $-1.5(14)$ \\
\hline $\mathrm{C}(54)-\mathrm{C}(55)-\mathrm{C}(56)-\mathrm{C}(51)$ & $3.3(13)$ \\
\hline$C(52)-C(51)-C(56)-C(55)$ & $-3.3(11)$ \\
\hline $\mathrm{P}(2)-\mathrm{C}(51)-\mathrm{C}(56)-\mathrm{C}(55)$ & $-179.7(6)$ \\
\hline $\mathrm{C}(71)-\mathrm{P}(3)-\mathrm{C}(61)-\mathrm{C}(62)$ & $-18.8(7)$ \\
\hline $\mathrm{P}(2)-\mathrm{P}(3)-\mathrm{C}(61)-\mathrm{C}(62)$ & $-115.6(6)$ \\
\hline $\mathrm{C}(71)-\mathrm{P}(3)-\mathrm{C}(61)-\mathrm{C}(66)$ & $168.1(6)$ \\
\hline $\mathrm{P}(2)-\mathrm{P}(3)-\mathrm{C}(61)-\mathrm{C}(66)$ & $71.3(6)$ \\
\hline $\mathrm{C}(66)-\mathrm{C}(61)-\mathrm{C}(62)-\mathrm{C}(63)$ & $0.5(11)$ \\
\hline $\mathrm{P}(3)-\mathrm{C}(61)-\mathrm{C}(62)-\mathrm{C}(63)$ & $-172.8(6)$ \\
\hline $\mathrm{C}(61)-\mathrm{C}(62)-\mathrm{C}(63)-\mathrm{C}(64)$ & $0.6(12)$ \\
\hline $\mathrm{C}(62)-\mathrm{C}(63)-\mathrm{C}(64)-\mathrm{C}(65)$ & $-0.8(12)$ \\
\hline $\mathrm{C}(63)-\mathrm{C}(64)-\mathrm{C}(65)-\mathrm{C}(66)$ & $0.0(11)$ \\
\hline $\mathrm{C}(64)-\mathrm{C}(65)-\mathrm{C}(66)-\mathrm{C}(61)$ & $1.1(11)$ \\
\hline $\mathrm{C}(62)-\mathrm{C}(61)-\mathrm{C}(66)-\mathrm{C}(65)$ & $-1.4(10)$ \\
\hline $\mathrm{P}(3)-\mathrm{C}(61)-\mathrm{C}(66)-\mathrm{C}(65)$ & $172.2(5)$ \\
\hline $\mathrm{C}(61)-\mathrm{P}(3)-\mathrm{C}(71)-\mathrm{C}(76)$ & $126.1(7)$ \\
\hline $\mathrm{P}(2)-\mathrm{P}(3)-\mathrm{C}(71)-\mathrm{C}(76)$ & $-132.5(6)$ \\
\hline $\mathrm{C}(61)-\mathrm{P}(3)-\mathrm{C}(71)-\mathrm{C}(72)$ & $-56.7(8)$ \\
\hline $\mathrm{P}(2)-\mathrm{P}(3)-\mathrm{C}(71)-\mathrm{C}(72)$ & $44.7(7)$ \\
\hline $\mathrm{C}(76)-\mathrm{C}(71)-\mathrm{C}(72)-\mathrm{C}(73)$ & $6.4(13)$ \\
\hline $\mathrm{P}(3)-\mathrm{C}(71)-\mathrm{C}(72)-\mathrm{C}(73)$ & $-170.9(7)$ \\
\hline $\mathrm{C}(71)-\mathrm{C}(72)-\mathrm{C}(73)-\mathrm{C}(74)$ & $-2.8(15)$ \\
\hline $\mathrm{C}(72)-\mathrm{C}(73)-\mathrm{C}(74)-\mathrm{C}(75)$ & $-1.9(17)$ \\
\hline $\mathrm{C}(73)-\mathrm{C}(74)-\mathrm{C}(75)-\mathrm{C}(76)$ & $2.8(18)$ \\
\hline $\mathrm{C}(72)-\mathrm{C}(71)-\mathrm{C}(76)-\mathrm{C}(75)$ & $-5.6(13)$ \\
\hline $\mathrm{P}(3)-\mathrm{C}(71)-\mathrm{C}(76)-\mathrm{C}(75)$ & $171.7(8)$ \\
\hline $\mathrm{C}(74)-\mathrm{C}(75)-\mathrm{C}(76)-\mathrm{C}(71)$ & $1.1(16)$ \\
\hline
\end{tabular}

Symmetry transformations used to generate equivalent atoms: 


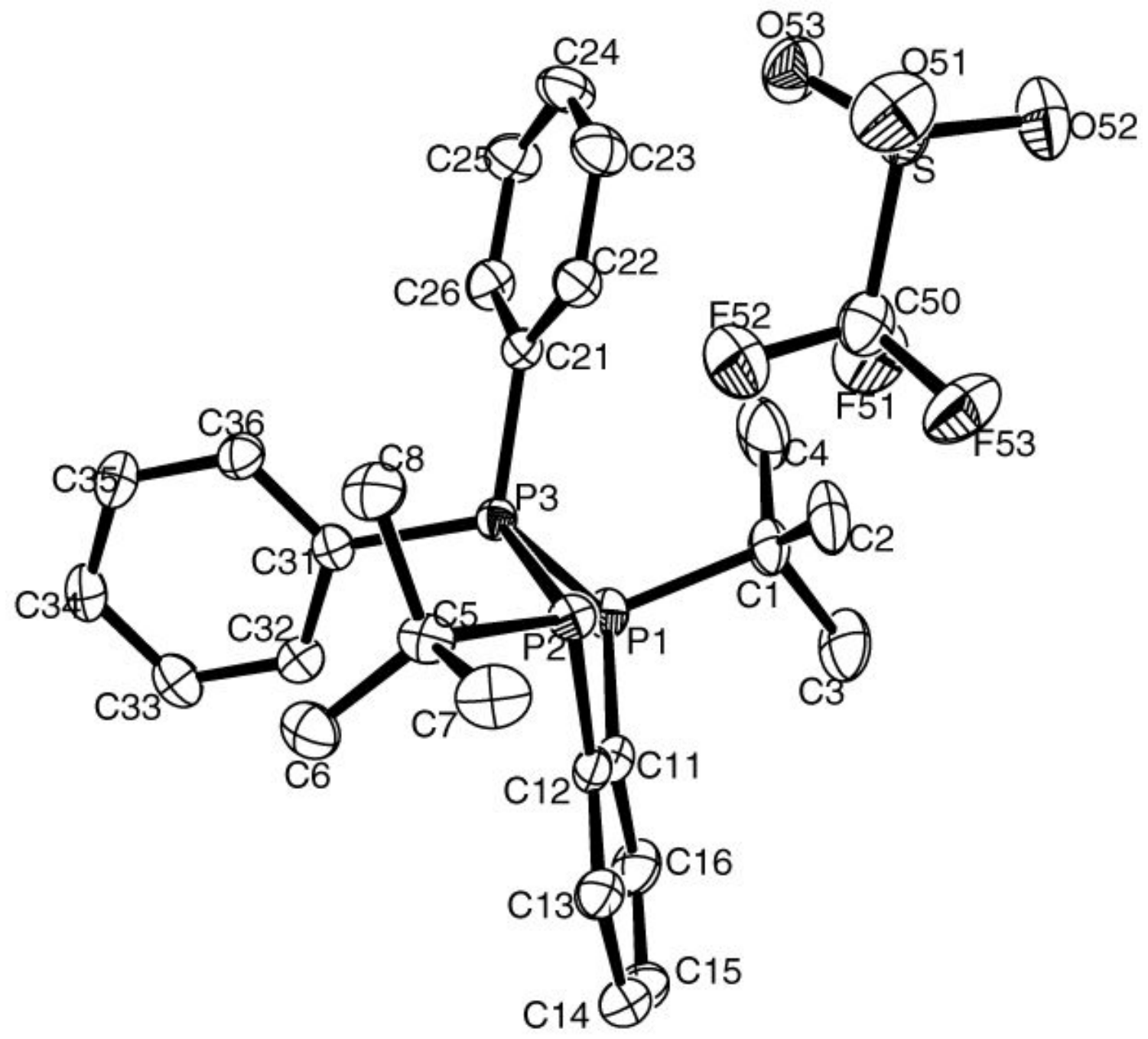

Figure S4: Solid state structure of $\mathbf{4 O S O}_{2} \mathbf{C F}_{3}$ including complete numbering scheme. Ellipsoides are $50 \%$ probability. Hydrogen atoms are removed for clarity and symmetry equivalent atoms are not shown. 
Table S20. Crystal data and structure refinement for $\mathbf{4 O S O}_{\mathbf{2}} \mathbf{C F}_{\mathbf{3}}$.

Identification code

Empirical formula

Formula weight

Temperature

Wavelength

Crystal system

Space group

Unit cell dimensions

Volume

$\mathrm{Z}$

Density (calculated)

Absorption coefficient

$\mathrm{F}(000)$

Crystal size

Theta range for data collection

Index ranges

Reflections collected

Independent reflections

Completeness to theta $=26.38^{\circ}$

Absorption correction

Max. and min. transmission

Refinement method

Data / restraints / parameters

Goodness-of-fit on $\mathrm{F}^{2}$

Final R indices [I $>2 \operatorname{sigma}(\mathrm{I})]$

$\mathrm{R}$ indices (all data)

Largest diff. peak and hole dal0310

C27 H32 F3 O3 P3 S

586.50

193(2) K

$0.71073 \AA$

Triclinic

P-1

$\begin{array}{ll}\mathrm{a}=10.0399(15) \AA & \alpha=102.753(3)^{\circ} . \\ \mathrm{b}=10.6383(16) \AA & \beta=107.407(3)^{\circ} . \\ \mathrm{c}=14.648(2) \AA & \gamma=100.374(3)^{\circ} .\end{array}$

1404.2(4) $\AA^{3}$

2

$1.387 \mathrm{Mg} / \mathrm{m}^{3}$

$0.334 \mathrm{~mm}^{-1}$

612

$0.31 \times 0.14 \times 0.09 \mathrm{~mm}^{3}$

1.52 to $26.38^{\circ}$.

$-12<=\mathrm{h}<=12,-13<=\mathrm{k}<=13,-18<=1<=10$

8007

$5620[\mathrm{R}(\mathrm{int})=0.0303]$

$97.7 \%$

Integration

0.9706 and 0.9036

Full-matrix least-squares on $\mathrm{F}^{2}$

$5620 / 0 / 334$

1.024

$\mathrm{R} 1=0.0508, \mathrm{wR} 2=0.1055$

$\mathrm{R} 1=0.0835, \mathrm{wR} 2=0.1179$

0.610 and -0.283 e. $\AA^{-3}$ 
Table S21. Atomic coordinates ( $\left.\mathrm{x} 10^{4}\right)$ and equivalent isotropic displacement parameters $\left(\AA^{2} \times 10^{3}\right)$ for $\mathbf{4 O S O}_{2} \mathbf{C F}_{3}$. U(eq) is defined as one third of the trace of the orthogonalized $\mathrm{U}^{\mathrm{ij}}$ tensor.

\begin{tabular}{|c|c|c|c|c|}
\hline & $\mathrm{x}$ & $\mathrm{y}$ & $\mathrm{z}$ & $\mathrm{U}(\mathrm{eq})$ \\
\hline $\mathrm{P}(1)$ & $1020(1)$ & $3865(1)$ & $829(1)$ & $24(1)$ \\
\hline $\mathrm{P}(2)$ & $1278(1)$ & $2212(1)$ & $2560(1)$ & $21(1)$ \\
\hline $\mathrm{P}(3)$ & $2317(1)$ & $4102(1)$ & 2391(1) & $19(1)$ \\
\hline $\mathrm{C}(1)$ & $1645(3)$ & 2626(3) & $11(2)$ & $31(1)$ \\
\hline$C(2)$ & $2008(4)$ & $1468(3)$ & $372(2)$ & $43(1)$ \\
\hline$C(3)$ & $358(4)$ & 2089(4) & $-986(2)$ & $48(1)$ \\
\hline$C(4)$ & 2941(4) & $3446(4)$ & $-135(3)$ & $55(1)$ \\
\hline$C(5)$ & $1287(3)$ & $2535(3)$ & $3884(2)$ & $25(1)$ \\
\hline$C(6)$ & 391(3) & $3455(3)$ & $4179(2)$ & $33(1)$ \\
\hline$C(7)$ & $753(4)$ & $1144(3)$ & $3987(2)$ & $37(1)$ \\
\hline $\mathrm{C}(8)$ & $2885(3)$ & $3099(3)$ & $4560(2)$ & $33(1)$ \\
\hline $\mathrm{C}(11)$ & $-570(3)$ & 2869(3) & $959(2)$ & $23(1)$ \\
\hline$C(12)$ & $-493(3)$ & $2230(3)$ & $1716(2)$ & $23(1)$ \\
\hline$C(13)$ & $-1769(3)$ & $1502(3)$ & $1746(2)$ & $29(1)$ \\
\hline$C(14)$ & $-3097(3)$ & $1422(3)$ & $1058(2)$ & $34(1)$ \\
\hline$C(15)$ & $-3189(3)$ & 2063(3) & $331(2)$ & $36(1)$ \\
\hline$C(16)$ & $-1927(3)$ & 2791(3) & $282(2)$ & $33(1)$ \\
\hline $\mathrm{C}(21)$ & $4210(3)$ & $4285(2)$ & $2598(2)$ & $21(1)$ \\
\hline $\mathrm{C}(22)$ & $4895(3)$ & $3339(3)$ & $2890(2)$ & $25(1)$ \\
\hline$C(23)$ & $6335(3)$ & $3490(3)$ & $3020(2)$ & $32(1)$ \\
\hline$C(24)$ & 7097(3) & $4587(3)$ & $2846(2)$ & $34(1)$ \\
\hline$C(25)$ & $6422(3)$ & $5528(3)$ & $2554(2)$ & $31(1)$ \\
\hline$C(26)$ & 4978(3) & $5393(3)$ & $2433(2)$ & $27(1)$ \\
\hline$C(31)$ & $2130(3)$ & $5627(2)$ & $3122(2)$ & $21(1)$ \\
\hline$C(32)$ & $831(3)$ & $5982(3)$ & $2845(2)$ & $24(1)$ \\
\hline$C(33)$ & $685(3)$ & $7126(3)$ & $3437(2)$ & $30(1)$ \\
\hline$C(34)$ & $1816(3)$ & 7901(3) & $4298(2)$ & $30(1)$ \\
\hline$C(35)$ & $3105(3)$ & $7552(3)$ & $4563(2)$ & $34(1)$ \\
\hline$C(36)$ & $3267(3)$ & 6412(3) & $3981(2)$ & $27(1)$ \\
\hline S & $6081(1)$ & $-777(1)$ & 2973(1) & $29(1)$ \\
\hline $\mathrm{F}(51)$ & $3863(2)$ & $-94(2)$ & 1919(2) & $50(1)$ \\
\hline
\end{tabular}




\begin{tabular}{lrrrr}
$\mathrm{F}(52)$ & $3935(2)$ & $-19(2)$ & $3406(2)$ & $53(1)$ \\
$\mathrm{F}(53)$ & $3281(2)$ & $-1914(2)$ & $2283(2)$ & $49(1)$ \\
$\mathrm{O}(51)$ & $6246(3)$ & $-1430(2)$ & $3740(2)$ & $46(1)$ \\
$\mathrm{O}(52)$ & $6135(3)$ & $-1536(2)$ & $2051(2)$ & $47(1)$ \\
$\mathrm{O}(53)$ & $6868(2)$ & $611(2)$ & $3307(2)$ & $38(1)$ \\
$\mathrm{C}(50)$ & $4203(3)$ & $-696(3)$ & $2627(2)$ & $32(1)$ \\
\hline
\end{tabular}


Table S22. Bond lengths $[\AA]$ and angles $\left[{ }^{\circ}\right]$ for $\mathbf{4 O S O}_{2} \mathbf{C F}_{3}$.

\begin{tabular}{|c|c|}
\hline $\mathrm{P}(1)-\mathrm{C}(11)$ & $1.835(3)$ \\
\hline $\mathrm{P}(1)-\mathrm{C}(1)$ & $1.901(3)$ \\
\hline$P(1)-P(3)$ & $2.1993(11)$ \\
\hline $\mathrm{P}(2)-\mathrm{C}(12)$ & $1.844(3)$ \\
\hline$P(2)-C(5)$ & $1.891(3)$ \\
\hline $\mathrm{P}(2)-\mathrm{P}(3)$ & $2.1933(10)$ \\
\hline $\mathrm{P}(3)-\mathrm{C}(21)$ & $1.800(3)$ \\
\hline $\mathrm{P}(3)-\mathrm{C}(31)$ & $1.810(3)$ \\
\hline$C(1)-C(2)$ & $1.505(4)$ \\
\hline $\mathrm{C}(1)-\mathrm{C}(4)$ & $1.529(4)$ \\
\hline$C(1)-C(3)$ & $1.538(4)$ \\
\hline$C(5)-C(6)$ & $1.523(4)$ \\
\hline $\mathrm{C}(5)-\mathrm{C}(8)$ & $1.534(4)$ \\
\hline$C(5)-C(7)$ & $1.536(4)$ \\
\hline$C(11)-C(16)$ & $1.401(4)$ \\
\hline$C(11)-C(12)$ & $1.413(4)$ \\
\hline $\mathrm{C}(12)-\mathrm{C}(13)$ & $1.390(4)$ \\
\hline$C(13)-C(14)$ & $1.385(4)$ \\
\hline $\mathrm{C}(14)-\mathrm{C}(15)$ & $1.376(5)$ \\
\hline$C(15)-C(16)$ & $1.391(4)$ \\
\hline$C(21)-C(22)$ & $1.389(4)$ \\
\hline$C(21)-C(26)$ & $1.398(4)$ \\
\hline$C(22)-C(23)$ & $1.375(4)$ \\
\hline$C(23)-C(24)$ & $1.392(4)$ \\
\hline$C(24)-C(25)$ & $1.379(4)$ \\
\hline$C(25)-C(26)$ & $1.383(4)$ \\
\hline$C(31)-C(36)$ & $1.384(4)$ \\
\hline $\mathrm{C}(31)-\mathrm{C}(32)$ & $1.392(4)$ \\
\hline$C(32)-C(33)$ & $1.388(4)$ \\
\hline$C(33)-C(34)$ & $1.381(4)$ \\
\hline$C(34)-C(35)$ & $1.378(4)$ \\
\hline$C(35)-C(36)$ & $1.386(4)$ \\
\hline $\mathrm{S}-\mathrm{O}(51)$ & $1.430(2)$ \\
\hline $\mathrm{S}-\mathrm{O}(52)$ & $1.434(2)$ \\
\hline
\end{tabular}




\begin{tabular}{|c|c|}
\hline $\mathrm{S}-\mathrm{O}(53)$ & $1.440(2)$ \\
\hline $\mathrm{S}-\mathrm{C}(50)$ & $1.823(3)$ \\
\hline $\mathrm{F}(51)-\mathrm{C}(50)$ & $1.328(4)$ \\
\hline $\mathrm{F}(52)-\mathrm{C}(50)$ & $1.335(3)$ \\
\hline $\mathrm{F}(53)-\mathrm{C}(50)$ & $1.338(3)$ \\
\hline $\mathrm{C}(11)-\mathrm{P}(1)-\mathrm{C}(1)$ & $105.39(12)$ \\
\hline C(11)-P(1)-P(3) & $90.66(9)$ \\
\hline C(1)-P(1)-P(3) & $106.78(10)$ \\
\hline $\mathrm{C}(12)-\mathrm{P}(2)-\mathrm{C}(5)$ & $109.74(13)$ \\
\hline $\mathrm{C}(12)-\mathrm{P}(2)-\mathrm{P}(3)$ & $90.93(9)$ \\
\hline $\mathrm{C}(5)-\mathrm{P}(2)-\mathrm{P}(3)$ & $108.18(9)$ \\
\hline $\mathrm{C}(21)-\mathrm{P}(3)-\mathrm{C}(31)$ & $108.09(12)$ \\
\hline C(21)-P(3)-P(2) & $112.26(9)$ \\
\hline C(31)-P(3)-P(2) & $117.44(9)$ \\
\hline C(21)-P(3)-P(1) & 113.07(9) \\
\hline C(31)-P(3)-P(1) & $105.69(10)$ \\
\hline $\mathrm{P}(2)-\mathrm{P}(3)-\mathrm{P}(1)$ & $100.01(4)$ \\
\hline $\mathrm{C}(2)-\mathrm{C}(1)-\mathrm{C}(4)$ & $110.9(3)$ \\
\hline $\mathrm{C}(2)-\mathrm{C}(1)-\mathrm{C}(3)$ & $109.0(3)$ \\
\hline C(4)-C(1)-C(3) & $109.7(3)$ \\
\hline $\mathrm{C}(2)-\mathrm{C}(1)-\mathrm{P}(1)$ & $117.1(2)$ \\
\hline $\mathrm{C}(4)-\mathrm{C}(1)-\mathrm{P}(1)$ & $106.2(2)$ \\
\hline $\mathrm{C}(3)-\mathrm{C}(1)-\mathrm{P}(1)$ & $103.5(2)$ \\
\hline$C(6)-C(5)-C(8)$ & $110.7(2)$ \\
\hline$C(6)-C(5)-C(7)$ & $110.7(2)$ \\
\hline $\mathrm{C}(8)-\mathrm{C}(5)-\mathrm{C}(7)$ & $107.9(2)$ \\
\hline $\mathrm{C}(6)-\mathrm{C}(5)-\mathrm{P}(2)$ & $116.6(2)$ \\
\hline $\mathrm{C}(8)-\mathrm{C}(5)-\mathrm{P}(2)$ & $105.52(19)$ \\
\hline $\mathrm{C}(7)-\mathrm{C}(5)-\mathrm{P}(2)$ & $104.89(19)$ \\
\hline$C(16)-C(11)-C(12)$ & 119.2(3) \\
\hline $\mathrm{C}(16)-\mathrm{C}(11)-\mathrm{P}(1)$ & $117.0(2)$ \\
\hline $\mathrm{C}(12)-\mathrm{C}(11)-\mathrm{P}(1)$ & $123.7(2)$ \\
\hline$C(13)-C(12)-C(11)$ & $118.9(3)$ \\
\hline $\mathrm{C}(13)-\mathrm{C}(12)-\mathrm{P}(2)$ & $120.6(2)$ \\
\hline $\mathrm{C}(11)-\mathrm{C}(12)-\mathrm{P}(2)$ & $120.2(2)$ \\
\hline
\end{tabular}




$\begin{array}{ll}\mathrm{C}(14)-\mathrm{C}(13)-\mathrm{C}(12) & 120.7(3) \\ \mathrm{C}(15)-\mathrm{C}(14)-\mathrm{C}(13) & 120.9(3) \\ \mathrm{C}(14)-\mathrm{C}(15)-\mathrm{C}(16) & 119.4(3) \\ \mathrm{C}(15)-\mathrm{C}(16)-\mathrm{C}(11) & 120.7(3) \\ \mathrm{C}(22)-\mathrm{C}(21)-\mathrm{C}(26) & 120.2(3) \\ \mathrm{C}(22)-\mathrm{C}(21)-\mathrm{P}(3) & 121.5(2) \\ \mathrm{C}(26)-\mathrm{C}(21)-\mathrm{P}(3) & 118.3(2) \\ \mathrm{C}(23)-\mathrm{C}(22)-\mathrm{C}(21) & 120.2(3) \\ \mathrm{C}(22)-\mathrm{C}(23)-\mathrm{C}(24) & 119.6(3) \\ \mathrm{C}(25)-\mathrm{C}(24)-\mathrm{C}(23) & 120.5(3) \\ \mathrm{C}(24)-\mathrm{C}(25)-\mathrm{C}(26) & 120.3(3) \\ \mathrm{C}(25)-\mathrm{C}(26)-\mathrm{C}(21) & 119.2(3) \\ \mathrm{C}(36)-\mathrm{C}(31)-\mathrm{C}(32) & 120.1(2) \\ \mathrm{C}(36)-\mathrm{C}(31)-\mathrm{P}(3) & 119.6(2) \\ \mathrm{C}(32)-\mathrm{C}(31)-\mathrm{P}(3) & 120.2(2) \\ \mathrm{C}(33)-\mathrm{C}(32)-\mathrm{C}(31) & 119.5(3) \\ \mathrm{C}(34)-\mathrm{C}(33)-\mathrm{C}(32) & 120.3(3) \\ \mathrm{C}(35)-\mathrm{C}(34)-\mathrm{C}(33) & 119.9(3) \\ \mathrm{C}(34)-\mathrm{C}(35)-\mathrm{C}(36) & 120.4(3) \\ \mathrm{C}(31)-\mathrm{C}(36)-\mathrm{C}(35) & 119.7(3) \\ \mathrm{O}(51)-\mathrm{S}-\mathrm{O}(52) & 115.21(15) \\ \mathrm{O}(51)-\mathrm{S}-\mathrm{O}(53) & 115.03(14) \\ \mathrm{O}(52)-\mathrm{S}-\mathrm{O}(53) & 114.94(14) \\ \mathrm{O}(51)-\mathrm{S}-\mathrm{C}(50) & 103.74(15) \\ \mathrm{O}(52)-\mathrm{S}-\mathrm{C}(50) & 102.86(14) \\ \mathrm{O}(53)-\mathrm{S}-\mathrm{C}(50) & 102.54(13) \\ \mathrm{F}(51)-\mathrm{C}(50)-\mathrm{F}(52) & 107.0(2) \\ \mathrm{F}(51)-\mathrm{C}(50)-\mathrm{F}(53) & 107.2(2) \\ \mathrm{F}(52)-\mathrm{C}(50)-\mathrm{F}(53) & 107.0(3) \\ \mathrm{F}(51)-\mathrm{C}(50)-\mathrm{S} & 111.8(2) \\ \mathrm{F}(52)-\mathrm{C}(50)-\mathrm{S} & 111.8(2) \\ \mathrm{F}(53)-\mathrm{C}(50)-\mathrm{S} & \end{array}$

Symmetry transformations used to generate equivalent atoms: 
Table S23. Anisotropic displacement parameters $\left(\AA^{2} \times 10^{3}\right)$ for $\mathbf{4 O S O}_{2} \mathbf{C F}_{3}$. The anisotropic displacement factor exponent takes the form: $-2 \pi^{2}\left[h^{2} a^{* 2} U^{11}+\ldots+2 h k a^{*} b^{*} U^{12}\right]$

\begin{tabular}{|c|c|c|c|c|c|c|}
\hline & $\mathrm{U}^{11}$ & $U^{22}$ & $\mathrm{U}^{33}$ & $\mathrm{U}^{23}$ & $\mathrm{U}^{13}$ & $\mathrm{U}^{12}$ \\
\hline $\mathrm{P}(1)$ & $30(1)$ & $24(1)$ & 19(1) & $8(1)$ & $7(1)$ & $9(1)$ \\
\hline $\mathrm{P}(2)$ & $22(1)$ & 21(1) & 21(1) & $9(1)$ & $7(1)$ & $6(1)$ \\
\hline $\mathrm{P}(3)$ & $20(1)$ & $19(1)$ & $18(1)$ & $5(1)$ & $6(1)$ & $6(1)$ \\
\hline$C(1)$ & $40(2)$ & $33(2)$ & 19(2) & $2(1)$ & $12(1)$ & $12(1)$ \\
\hline$C(2)$ & $61(2)$ & $40(2)$ & $25(2)$ & $1(1)$ & $12(2)$ & $28(2)$ \\
\hline$C(3)$ & $64(2)$ & $53(2)$ & 21(2) & $5(2)$ & $9(2)$ & $20(2)$ \\
\hline$C(4)$ & 71(3) & $53(2)$ & $49(2)$ & $5(2)$ & $42(2)$ & $13(2)$ \\
\hline$C(5)$ & $24(2)$ & $34(2)$ & $22(2)$ & $14(1)$ & $8(1)$ & $10(1)$ \\
\hline$C(6)$ & $33(2)$ & $46(2)$ & $25(2)$ & $14(1)$ & $13(1)$ & $15(1)$ \\
\hline$C(7)$ & $37(2)$ & $42(2)$ & $38(2)$ & $26(2)$ & $13(2)$ & $9(2)$ \\
\hline$C(8)$ & $32(2)$ & $46(2)$ & $21(2)$ & $14(1)$ & $6(1)$ & $10(1)$ \\
\hline$C(11)$ & $26(2)$ & 21(1) & $20(1)$ & $3(1)$ & $5(1)$ & $5(1)$ \\
\hline$C(12)$ & 21(1) & 21(1) & $20(1)$ & $-1(1)$ & $3(1)$ & $6(1)$ \\
\hline$C(13)$ & $26(2)$ & $31(2)$ & $24(2)$ & $3(1)$ & $8(1)$ & $4(1)$ \\
\hline$C(14)$ & $25(2)$ & $37(2)$ & $32(2)$ & $0(1)$ & $8(1)$ & $4(1)$ \\
\hline$C(15)$ & $23(2)$ & $43(2)$ & $31(2)$ & $1(2)$ & $-1(1)$ & $10(1)$ \\
\hline$C(16)$ & $35(2)$ & $35(2)$ & $22(2)$ & $5(1)$ & 2(1) & $10(1)$ \\
\hline$C(21)$ & 19(1) & $22(1)$ & 19(1) & $3(1)$ & $5(1)$ & $6(1)$ \\
\hline$C(22)$ & $26(2)$ & 21(1) & $29(2)$ & $9(1)$ & $12(1)$ & $6(1)$ \\
\hline$C(23)$ & $31(2)$ & $29(2)$ & $40(2)$ & 13(1) & 13(1) & $14(1)$ \\
\hline$C(24)$ & $25(2)$ & $36(2)$ & $46(2)$ & $13(2)$ & $18(2)$ & $9(1)$ \\
\hline$C(25)$ & $28(2)$ & $29(2)$ & $40(2)$ & $14(1)$ & $16(1)$ & $5(1)$ \\
\hline$C(26)$ & $31(2)$ & $20(1)$ & $31(2)$ & $10(1)$ & $10(1)$ & $7(1)$ \\
\hline$C(31)$ & $25(2)$ & $20(1)$ & $22(1)$ & $8(1)$ & 11(1) & $7(1)$ \\
\hline$C(32)$ & $25(2)$ & $24(1)$ & $23(2)$ & $9(1)$ & $7(1)$ & $6(1)$ \\
\hline$C(33)$ & $36(2)$ & $30(2)$ & $33(2)$ & 13(1) & 18(1) & $16(1)$ \\
\hline$C(34)$ & $40(2)$ & $24(1)$ & $28(2)$ & $4(1)$ & $16(1)$ & $10(1)$ \\
\hline$C(35)$ & $37(2)$ & $32(2)$ & $24(2)$ & $-1(1)$ & $7(1)$ & $5(1)$ \\
\hline$C(36)$ & $24(2)$ & $27(1)$ & $25(2)$ & $5(1)$ & $4(1)$ & $9(1)$ \\
\hline S & $36(1)$ & $24(1)$ & $26(1)$ & $7(1)$ & $10(1)$ & $7(1)$ \\
\hline $\mathrm{F}(51)$ & $58(1)$ & $44(1)$ & $45(1)$ & $22(1)$ & $4(1)$ & $18(1)$ \\
\hline
\end{tabular}




\begin{tabular}{lllllll}
$\mathrm{F}(52)$ & $51(1)$ & $56(1)$ & $52(1)$ & $3(1)$ & $28(1)$ & $15(1)$ \\
$\mathrm{F}(53)$ & $40(1)$ & $36(1)$ & $53(1)$ & $10(1)$ & $2(1)$ & $-6(1)$ \\
$\mathrm{O}(51)$ & $52(2)$ & $45(1)$ & $41(1)$ & $27(1)$ & $6(1)$ & $12(1)$ \\
$\mathrm{O}(52)$ & $65(2)$ & $40(1)$ & $38(1)$ & $3(1)$ & $24(1)$ & $22(1)$ \\
$\mathrm{O}(53)$ & $39(1)$ & $29(1)$ & $42(1)$ & $6(1)$ & $17(1)$ & $-1(1)$ \\
$\mathrm{C}(50)$ & $38(2)$ & $25(2)$ & $32(2)$ & $9(1)$ & $10(1)$ & $9(1)$ \\
\hline
\end{tabular}


Table S24. Torsion angles $\left[{ }^{\circ}\right]$ for $\mathbf{4 O S O}_{2} \mathbf{C F}_{3}$.

\begin{tabular}{|c|c|}
\hline $\mathrm{C}(12)-\mathrm{P}(2)-\mathrm{P}(3)-\mathrm{C}(21)$ & $-153.98(12)$ \\
\hline $\mathrm{C}(5)-\mathrm{P}(2)-\mathrm{P}(3)-\mathrm{C}(21)$ & $94.87(13)$ \\
\hline $\mathrm{C}(12)-\mathrm{P}(2)-\mathrm{P}(3)-\mathrm{C}(31)$ & $79.82(14)$ \\
\hline $\mathrm{C}(5)-\mathrm{P}(2)-\mathrm{P}(3)-\mathrm{C}(31)$ & $-31.34(15)$ \\
\hline $\mathrm{C}(12)-\mathrm{P}(2)-\mathrm{P}(3)-\mathrm{P}(1)$ & $-33.84(9)$ \\
\hline $\mathrm{C}(5)-\mathrm{P}(2)-\mathrm{P}(3)-\mathrm{P}(1)$ & $-144.99(10)$ \\
\hline$C(11)-P(1)-P(3)-C(21)$ & $150.71(13)$ \\
\hline $\mathrm{C}(1)-\mathrm{P}(1)-\mathrm{P}(3)-\mathrm{C}(21)$ & $44.41(14)$ \\
\hline $\mathrm{C}(11)-\mathrm{P}(1)-\mathrm{P}(3)-\mathrm{C}(31)$ & $-91.24(13)$ \\
\hline $\mathrm{C}(1)-\mathrm{P}(1)-\mathrm{P}(3)-\mathrm{C}(31)$ & $162.46(13)$ \\
\hline $\mathrm{C}(11)-\mathrm{P}(1)-\mathrm{P}(3)-\mathrm{P}(2)$ & $31.15(9)$ \\
\hline $\mathrm{C}(1)-\mathrm{P}(1)-\mathrm{P}(3)-\mathrm{P}(2)$ & $-75.15(10)$ \\
\hline $\mathrm{C}(11)-\mathrm{P}(1)-\mathrm{C}(1)-\mathrm{C}(2)$ & $-57.3(3)$ \\
\hline $\mathrm{P}(3)-\mathrm{P}(1)-\mathrm{C}(1)-\mathrm{C}(2)$ & $38.2(3)$ \\
\hline $\mathrm{C}(11)-\mathrm{P}(1)-\mathrm{C}(1)-\mathrm{C}(4)$ & $178.2(2)$ \\
\hline $\mathrm{P}(3)-\mathrm{P}(1)-\mathrm{C}(1)-\mathrm{C}(4)$ & $-86.4(2)$ \\
\hline $\mathrm{C}(11)-\mathrm{P}(1)-\mathrm{C}(1)-\mathrm{C}(3)$ & $62.7(2)$ \\
\hline $\mathrm{P}(3)-\mathrm{P}(1)-\mathrm{C}(1)-\mathrm{C}(3)$ & $158.17(19)$ \\
\hline $\mathrm{C}(12)-\mathrm{P}(2)-\mathrm{C}(5)-\mathrm{C}(6)$ & $-32.7(2)$ \\
\hline $\mathrm{P}(3)-\mathrm{P}(2)-\mathrm{C}(5)-\mathrm{C}(6)$ & $65.1(2)$ \\
\hline $\mathrm{C}(12)-\mathrm{P}(2)-\mathrm{C}(5)-\mathrm{C}(8)$ & $-155.99(19)$ \\
\hline $\mathrm{P}(3)-\mathrm{P}(2)-\mathrm{C}(5)-\mathrm{C}(8)$ & $-58.2(2)$ \\
\hline $\mathrm{C}(12)-\mathrm{P}(2)-\mathrm{C}(5)-\mathrm{C}(7)$ & $90.2(2)$ \\
\hline $\mathrm{P}(3)-\mathrm{P}(2)-\mathrm{C}(5)-\mathrm{C}(7)$ & $-171.96(17)$ \\
\hline $\mathrm{C}(1)-\mathrm{P}(1)-\mathrm{C}(11)-\mathrm{C}(16)$ & $-94.1(2)$ \\
\hline $\mathrm{P}(3)-\mathrm{P}(1)-\mathrm{C}(11)-\mathrm{C}(16)$ & $158.3(2)$ \\
\hline $\mathrm{C}(1)-\mathrm{P}(1)-\mathrm{C}(11)-\mathrm{C}(12)$ & $88.1(2)$ \\
\hline $\mathrm{P}(3)-\mathrm{P}(1)-\mathrm{C}(11)-\mathrm{C}(12)$ & $-19.5(2)$ \\
\hline $\mathrm{C}(16)-\mathrm{C}(11)-\mathrm{C}(12)-\mathrm{C}(13)$ & $2.1(4)$ \\
\hline$P(1)-C(11)-C(12)-C(13)$ & $179.8(2)$ \\
\hline $\mathrm{C}(16)-\mathrm{C}(11)-\mathrm{C}(12)-\mathrm{P}(2)$ & $175.6(2)$ \\
\hline $\mathrm{P}(1)-\mathrm{C}(11)-\mathrm{C}(12)-\mathrm{P}(2)$ & $-6.7(3)$ \\
\hline $\mathrm{C}(5)-\mathrm{P}(2)-\mathrm{C}(12)-\mathrm{C}(13)$ & $-48.5(2)$ \\
\hline $\mathrm{P}(3)-\mathrm{P}(2)-\mathrm{C}(12)-\mathrm{C}(13)$ & $-158.3(2)$ \\
\hline
\end{tabular}




\begin{tabular}{|c|c|}
\hline $\mathrm{C}(5)-\mathrm{P}(2)-\mathrm{C}(12)-\mathrm{C}(11)$ & $138.1(2)$ \\
\hline $\mathrm{P}(3)-\mathrm{P}(2)-\mathrm{C}(12)-\mathrm{C}(11)$ & $28.4(2)$ \\
\hline $\mathrm{C}(11)-\mathrm{C}(12)-\mathrm{C}(13)-\mathrm{C}(14)$ & $-1.2(4)$ \\
\hline $\mathrm{P}(2)-\mathrm{C}(12)-\mathrm{C}(13)-\mathrm{C}(14)$ & $-174.6(2)$ \\
\hline$C(12)-C(13)-C(14)-C(15)$ & $-0.2(4)$ \\
\hline $\mathrm{C}(13)-\mathrm{C}(14)-\mathrm{C}(15)-\mathrm{C}(16)$ & $0.5(4)$ \\
\hline$C(14)-C(15)-C(16)-C(11)$ & $0.6(4)$ \\
\hline$C(12)-C(11)-C(16)-C(15)$ & $-1.9(4)$ \\
\hline $\mathrm{P}(1)-\mathrm{C}(11)-\mathrm{C}(16)-\mathrm{C}(15)$ & $-179.7(2)$ \\
\hline $\mathrm{C}(31)-\mathrm{P}(3)-\mathrm{C}(21)-\mathrm{C}(22)$ & $127.5(2)$ \\
\hline $\mathrm{P}(2)-\mathrm{P}(3)-\mathrm{C}(21)-\mathrm{C}(22)$ & $-3.6(2)$ \\
\hline $\mathrm{P}(1)-\mathrm{P}(3)-\mathrm{C}(21)-\mathrm{C}(22)$ & $-115.9(2)$ \\
\hline $\mathrm{C}(31)-\mathrm{P}(3)-\mathrm{C}(21)-\mathrm{C}(26)$ & $-54.1(2)$ \\
\hline $\mathrm{P}(2)-\mathrm{P}(3)-\mathrm{C}(21)-\mathrm{C}(26)$ & $174.79(18)$ \\
\hline $\mathrm{P}(1)-\mathrm{P}(3)-\mathrm{C}(21)-\mathrm{C}(26)$ & $62.5(2)$ \\
\hline$C(26)-C(21)-C(22)-C(23)$ & $0.2(4)$ \\
\hline $\mathrm{P}(3)-\mathrm{C}(21)-\mathrm{C}(22)-\mathrm{C}(23)$ & $178.5(2)$ \\
\hline $\mathrm{C}(21)-\mathrm{C}(22)-\mathrm{C}(23)-\mathrm{C}(24)$ & $-0.8(4)$ \\
\hline$C(22)-C(23)-C(24)-C(25)$ & $0.7(4)$ \\
\hline$C(23)-C(24)-C(25)-C(26)$ & $0.1(5)$ \\
\hline$C(24)-C(25)-C(26)-C(21)$ & $-0.7(4)$ \\
\hline$C(22)-C(21)-C(26)-C(25)$ & $0.6(4)$ \\
\hline $\mathrm{P}(3)-\mathrm{C}(21)-\mathrm{C}(26)-\mathrm{C}(25)$ & $-177.8(2)$ \\
\hline $\mathrm{C}(21)-\mathrm{P}(3)-\mathrm{C}(31)-\mathrm{C}(36)$ & $-26.5(3)$ \\
\hline $\mathrm{P}(2)-\mathrm{P}(3)-\mathrm{C}(31)-\mathrm{C}(36)$ & $101.7(2)$ \\
\hline $\mathrm{P}(1)-\mathrm{P}(3)-\mathrm{C}(31)-\mathrm{C}(36)$ & $-147.8(2)$ \\
\hline $\mathrm{C}(21)-\mathrm{P}(3)-\mathrm{C}(31)-\mathrm{C}(32)$ & $156.0(2)$ \\
\hline $\mathrm{P}(2)-\mathrm{P}(3)-\mathrm{C}(31)-\mathrm{C}(32)$ & $-75.8(2)$ \\
\hline $\mathrm{P}(1)-\mathrm{P}(3)-\mathrm{C}(31)-\mathrm{C}(32)$ & $34.7(2)$ \\
\hline $\mathrm{C}(36)-\mathrm{C}(31)-\mathrm{C}(32)-\mathrm{C}(33)$ & $-0.2(4)$ \\
\hline $\mathrm{P}(3)-\mathrm{C}(31)-\mathrm{C}(32)-\mathrm{C}(33)$ & $177.4(2)$ \\
\hline $\mathrm{C}(31)-\mathrm{C}(32)-\mathrm{C}(33)-\mathrm{C}(34)$ & $-0.5(4)$ \\
\hline $\mathrm{C}(32)-\mathrm{C}(33)-\mathrm{C}(34)-\mathrm{C}(35)$ & $1.2(4)$ \\
\hline $\mathrm{C}(33)-\mathrm{C}(34)-\mathrm{C}(35)-\mathrm{C}(36)$ & $-1.2(5)$ \\
\hline $\mathrm{C}(32)-\mathrm{C}(31)-\mathrm{C}(36)-\mathrm{C}(35)$ & $0.1(4)$ \\
\hline $\mathrm{P}(3)-\mathrm{C}(31)-\mathrm{C}(36)-\mathrm{C}(35)$ & $-177.4(2)$ \\
\hline
\end{tabular}




$\begin{array}{lc}\mathrm{C}(34)-\mathrm{C}(35)-\mathrm{C}(36)-\mathrm{C}(31) & 0.6(5) \\ \mathrm{O}(51)-\mathrm{S}-\mathrm{C}(50)-\mathrm{F}(51) & 179.28(19) \\ \mathrm{O}(52)-\mathrm{S}-\mathrm{C}(50)-\mathrm{F}(51) & -60.4(2) \\ \mathrm{O}(53)-\mathrm{S}-\mathrm{C}(50)-\mathrm{F}(51) & 59.2(2) \\ \mathrm{O}(51)-\mathrm{S}-\mathrm{C}(50)-\mathrm{F}(52) & 59.3(2) \\ \mathrm{O}(52)-\mathrm{S}-\mathrm{C}(50)-\mathrm{F}(52) & 179.7(2) \\ \mathrm{O}(53)-\mathrm{S}-\mathrm{C}(50)-\mathrm{F}(52) & -60.7(2) \\ \mathrm{O}(51)-\mathrm{S}-\mathrm{C}(50)-\mathrm{F}(53) & -60.5(2) \\ \mathrm{O}(52)-\mathrm{S}-\mathrm{C}(50)-\mathrm{F}(53) & 59.9(2) \\ \mathrm{O}(53)-\mathrm{S}-\mathrm{C}(50)-\mathrm{F}(53) & 179.4(2)\end{array}$

Symmetry transformations used to generate equivalent atoms: 


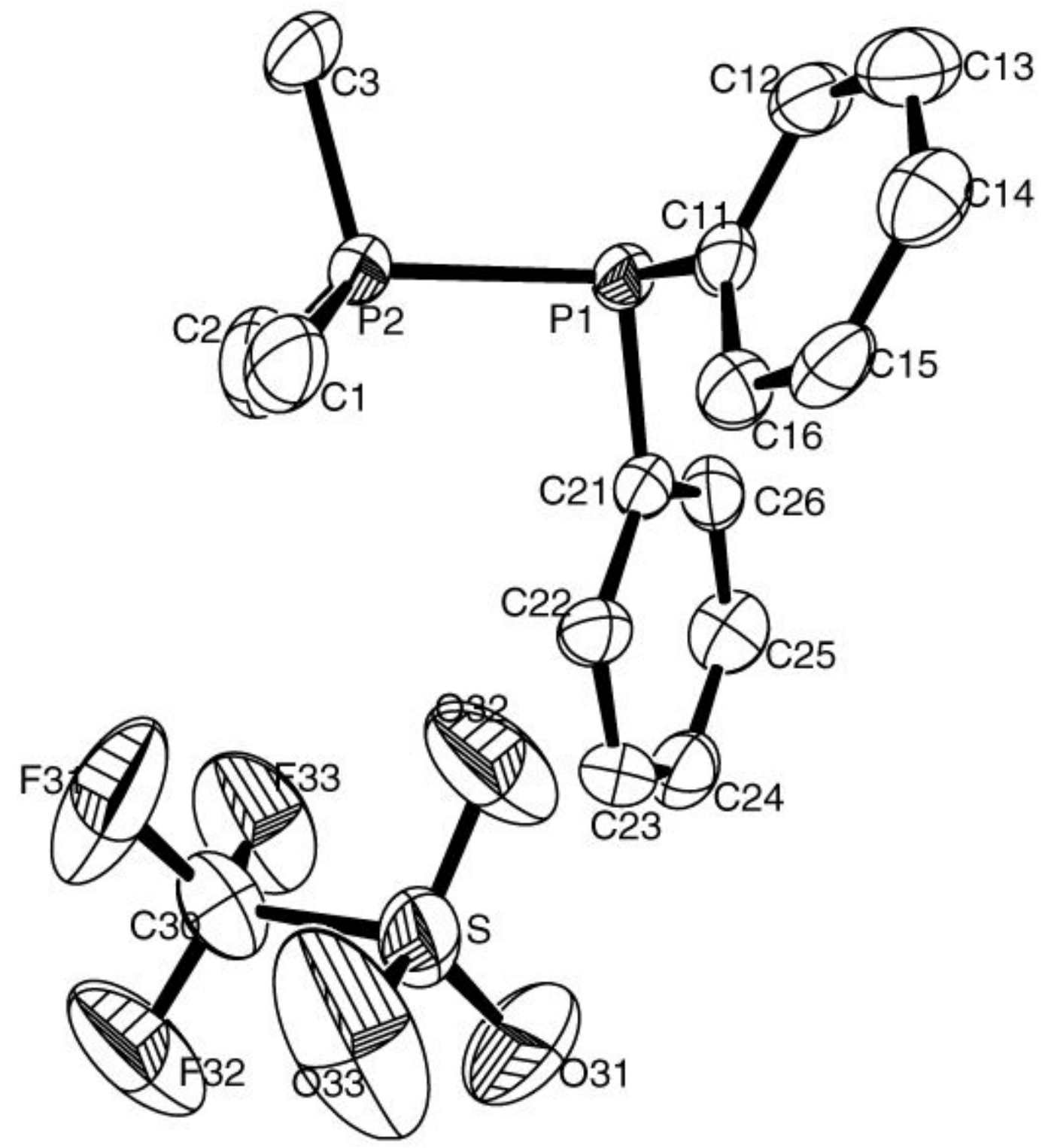

Figure S5: Soilid State structure of $\mathbf{2} \mathbf{d} \mathbf{O S O}_{2} \mathbf{C F}_{3}$ with full labeling scheme. Ellipsoids are $50 \%$ probability and hydrogen atoms are removed for clarity. 
Table S25. Crystal data and structure refinement for $\mathbf{2 d O S O}_{2} \mathbf{C F}_{3}$.

Identification code

Empirical formula

Formula weight

Temperature

Wavelength

Crystal system

Space group

Unit cell dimensions

Volume

$\mathrm{Z}$

Density (calculated)

Absorption coefficient

$\mathrm{F}(000)$

Crystal size

Theta range for data collection

Index ranges

Reflections collected

Independent reflections

Completeness to theta $=24.99^{\circ}$

Absorption correction

Max. and min. transmission

Refinement method

Data / restraints / parameters

Goodness-of-fit on $\mathrm{F}^{2}$

Final $\mathrm{R}$ indices [I $>2 \operatorname{sigma}(\mathrm{I})]$

$\mathrm{R}$ indices (all data)

Largest diff. peak and hole dal0321

C16 H19 F3 O3 P2 S

410.31

193(2) K

$0.71073 \AA$

Monoclinic

$\mathrm{P} 2{ }_{1} / \mathrm{n}$

$\mathrm{a}=11.569(3) \AA$ $\alpha=90^{\circ}$.

$\mathrm{b}=8.265(2) \AA$ $\beta=97.214(5)^{\circ}$.

$\mathrm{c}=19.965(5) \AA$ $\gamma=90^{\circ}$.

1893.9(8) $\AA^{3}$

4

$1.439 \mathrm{Mg} / \mathrm{m}^{3}$

$0.380 \mathrm{~mm}^{-1}$

848

$0.97 \times 0.08 \times 0.07 \mathrm{~mm}^{3}$

1.94 to $24.99^{\circ}$.

$-13<=\mathrm{h}<=12,-9<=\mathrm{k}<=9,-23<=\mathrm{k}<=19$

8289

$3325[\mathrm{R}(\mathrm{int})=0.1322]$

$100.0 \%$

Empirical

0.9739 and 0.7093

Full-matrix least-squares on $\mathrm{F}^{2}$

$3325 / 0 / 226$

1.038

$\mathrm{R} 1=0.0956, \mathrm{wR} 2=0.2113$

$\mathrm{R} 1=0.1424, \mathrm{wR} 2=0.2418$

0.771 and -0.485 e. $\AA^{-3}$ 
Table S26. Atomic coordinates ( $\left.\mathrm{x} 10^{4}\right)$ and equivalent isotropic displacement parameters $\left(\AA^{2} \times 10^{3}\right)$ for $2 \mathbf{d O S O}_{2} \mathbf{C F}_{3}$. U(eq) is defined as one third of the trace of the orthogonalized $\mathrm{U}^{\mathrm{ij}}$ tensor.

\begin{tabular}{|c|c|c|c|c|}
\hline & $\mathrm{x}$ & $\mathrm{y}$ & $\mathrm{z}$ & $\mathrm{U}(\mathrm{eq})$ \\
\hline $\mathrm{P}(1)$ & 2187(1) & $5825(2)$ & $-1872(1)$ & $35(1)$ \\
\hline $\mathrm{P}(2)$ & $2565(2)$ & $7275(2)$ & $-954(1)$ & $38(1)$ \\
\hline $\mathrm{C}(1)$ & $3476(7)$ & $6410(9)$ & $-257(3)$ & $63(2)$ \\
\hline$C(2)$ & $1215(7)$ & $7842(10)$ & $-688(4)$ & $75(2)$ \\
\hline $\mathrm{C}(3)$ & $3277(8)$ & $9066(8)$ & $-1196(4)$ & $69(2)$ \\
\hline$C(11)$ & $3538(5)$ & 4699(6) & $-1859(3)$ & $34(1)$ \\
\hline$C(12)$ & $4243(5)$ & $5137(7)$ & $-2354(3)$ & $42(1)$ \\
\hline$C(13)$ & $5252(6)$ & $4326(8)$ & $-2407(4)$ & $54(2)$ \\
\hline$C(14)$ & $5613(5)$ & $3068(8)$ & $-1983(3)$ & $47(2)$ \\
\hline$C(15)$ & 4942(5) & $2627(7)$ & $-1485(3)$ & $42(1)$ \\
\hline$C(16)$ & $3918(6)$ & $3430(7)$ & $-1425(3)$ & $44(2)$ \\
\hline$C(21)$ & $1075(5)$ & $4437(6)$ & $-1631(3)$ & $34(1)$ \\
\hline$C(22)$ & $1130(5)$ & $3495(7)$ & $-1045(3)$ & $41(1)$ \\
\hline$C(23)$ & $202(6)$ & $2525(7)$ & $-938(3)$ & $47(2)$ \\
\hline$C(24)$ & $-801(6)$ & $2499(7)$ & $-1401(3)$ & $48(2)$ \\
\hline$C(25)$ & $-853(5)$ & $3419(8)$ & $-1977(3)$ & $49(2)$ \\
\hline$C(26)$ & $83(5)$ & $4368(7)$ & $-2096(3)$ & $41(2)$ \\
\hline S & $3021(2)$ & $1418(2)$ & $681(1)$ & $66(1)$ \\
\hline $\mathrm{F}(31)$ & $3011(6)$ & $4175(7)$ & $1257(4)$ & $137(3)$ \\
\hline$F(32)$ & $1817(5)$ & $2621(8)$ & $1575(2)$ & $120(2)$ \\
\hline $\mathrm{F}(33)$ & 1531(5) & $3814(7)$ & $619(3)$ & $102(2)$ \\
\hline $\mathrm{O}(31)$ & $2069(11)$ & $379(10)$ & $461(4)$ & $198(6)$ \\
\hline $\mathrm{O}(32)$ & $3489(6)$ & 2131(8) & $142(3)$ & $96(2)$ \\
\hline $\mathrm{O}(33)$ & $3764(9)$ & $820(11)$ & $1233(4)$ & $169(5)$ \\
\hline $\mathrm{C}(30)$ & 2281(6) & $3048(10)$ & 1052(3) & $56(2)$ \\
\hline
\end{tabular}


Table S27. Bond lengths $[\AA]$ and angles $\left[^{\circ}\right]$ for $\mathbf{2} \mathbf{d O S O}_{2} \mathbf{C F}_{3}$.

\begin{tabular}{|c|c|}
\hline$P(1)-C(11)$ & $1.816(6)$ \\
\hline$P(1)-C(21)$ & $1.833(6)$ \\
\hline $\mathrm{P}(1)-\mathrm{P}(2)$ & $2.187(2)$ \\
\hline $\mathrm{P}(2)-\mathrm{C}(2)$ & $1.774(7)$ \\
\hline $\mathrm{P}(2)-\mathrm{C}(1)$ & $1.786(7)$ \\
\hline$P(2)-C(3)$ & $1.790(6)$ \\
\hline$C(11)-C(16)$ & $1.397(8)$ \\
\hline $\mathrm{C}(11)-\mathrm{C}(12)$ & $1.406(8)$ \\
\hline$C(12)-C(13)$ & $1.362(9)$ \\
\hline$C(13)-C(14)$ & $1.373(9)$ \\
\hline$C(14)-C(15)$ & $1.385(9)$ \\
\hline$C(15)-C(16)$ & $1.376(8)$ \\
\hline$C(21)-C(26)$ & $1.384(8)$ \\
\hline$C(21)-C(22)$ & $1.401(8)$ \\
\hline$C(22)-C(23)$ & $1.378(8)$ \\
\hline$C(23)-C(24)$ & $1.391(9)$ \\
\hline$C(24)-C(25)$ & $1.373(9)$ \\
\hline$C(25)-C(26)$ & $1.381(8)$ \\
\hline $\mathrm{S}-\mathrm{O}(32)$ & $1.394(5)$ \\
\hline $\mathrm{S}-\mathrm{O}(33)$ & $1.400(7)$ \\
\hline $\mathrm{S}-\mathrm{O}(31)$ & $1.422(9)$ \\
\hline $\mathrm{S}-\mathrm{C}(30)$ & $1.804(7)$ \\
\hline $\mathrm{F}(31)-\mathrm{C}(30)$ & $1.290(9)$ \\
\hline $\mathrm{F}(32)-\mathrm{C}(30)$ & $1.284(8)$ \\
\hline $\mathrm{F}(33)-\mathrm{C}(30)$ & $1.308(8)$ \\
\hline $\mathrm{C}(11)-\mathrm{P}(1)-\mathrm{C}(21)$ & 107.9(3) \\
\hline $\mathrm{C}(11)-\mathrm{P}(1)-\mathrm{P}(2)$ & $100.86(18)$ \\
\hline $\mathrm{C}(21)-\mathrm{P}(1)-\mathrm{P}(2)$ & $101.33(18)$ \\
\hline $\mathrm{C}(2)-\mathrm{P}(2)-\mathrm{C}(1)$ & $109.2(4)$ \\
\hline $\mathrm{C}(2)-\mathrm{P}(2)-\mathrm{C}(3)$ & $108.3(4)$ \\
\hline $\mathrm{C}(1)-\mathrm{P}(2)-\mathrm{C}(3)$ & 107.2(4) \\
\hline $\mathrm{C}(2)-\mathrm{P}(2)-\mathrm{P}(1)$ & $107.7(3)$ \\
\hline$C(1)-P(2)-P(1)$ & $118.0(2)$ \\
\hline
\end{tabular}




\begin{tabular}{|c|c|}
\hline $\mathrm{C}(3)-\mathrm{P}(2)-\mathrm{P}(1)$ & 106.1(2) \\
\hline$C(16)-C(11)-C(12)$ & $117.7(6)$ \\
\hline $\mathrm{C}(16)-\mathrm{C}(11)-\mathrm{P}(1)$ & $126.7(4)$ \\
\hline $\mathrm{C}(12)-\mathrm{C}(11)-\mathrm{P}(1)$ & $115.6(4)$ \\
\hline$C(13)-C(12)-C(11)$ & $120.4(6)$ \\
\hline$C(12)-C(13)-C(14)$ & $121.5(6)$ \\
\hline$C(13)-C(14)-C(15)$ & $119.2(6)$ \\
\hline$C(16)-C(15)-C(14)$ & $120.1(6)$ \\
\hline$C(15)-C(16)-C(11)$ & $121.1(6)$ \\
\hline$C(26)-C(21)-C(22)$ & $119.0(5)$ \\
\hline $\mathrm{C}(26)-\mathrm{C}(21)-\mathrm{P}(1)$ & $113.6(4)$ \\
\hline $\mathrm{C}(22)-\mathrm{C}(21)-\mathrm{P}(1)$ & $127.4(5)$ \\
\hline $\mathrm{C}(23)-\mathrm{C}(22)-\mathrm{C}(21)$ & $119.9(6)$ \\
\hline $\mathrm{C}(22)-\mathrm{C}(23)-\mathrm{C}(24)$ & $120.5(6)$ \\
\hline $\mathrm{C}(25)-\mathrm{C}(24)-\mathrm{C}(23)$ & $119.5(6)$ \\
\hline$C(24)-C(25)-C(26)$ & $120.4(6)$ \\
\hline $\mathrm{C}(25)-\mathrm{C}(26)-\mathrm{C}(21)$ & $120.6(6)$ \\
\hline $\mathrm{O}(32)-\mathrm{S}-\mathrm{O}(33)$ & $119.8(5)$ \\
\hline $\mathrm{O}(32)-\mathrm{S}-\mathrm{O}(31)$ & $112.2(5)$ \\
\hline $\mathrm{O}(33)-\mathrm{S}-\mathrm{O}(31)$ & $113.9(7)$ \\
\hline $\mathrm{O}(32)-\mathrm{S}-\mathrm{C}(30)$ & $104.5(4)$ \\
\hline $\mathrm{O}(33)-\mathrm{S}-\mathrm{C}(30)$ & $102.5(4)$ \\
\hline $\mathrm{O}(31)-\mathrm{S}-\mathrm{C}(30)$ & $100.9(5)$ \\
\hline $\mathrm{F}(32)-\mathrm{C}(30)-\mathrm{F}(31)$ & $105.3(7)$ \\
\hline $\mathrm{F}(32)-\mathrm{C}(30)-\mathrm{F}(33)$ & $111.0(6)$ \\
\hline $\mathrm{F}(31)-\mathrm{C}(30)-\mathrm{F}(33)$ & $102.4(7)$ \\
\hline $\mathrm{F}(32)-\mathrm{C}(30)-\mathrm{S}$ & $113.4(6)$ \\
\hline $\mathrm{F}(31)-\mathrm{C}(30)-\mathrm{S}$ & $110.2(5)$ \\
\hline$F(33)-C(30)-S$ & $113.6(5)$ \\
\hline
\end{tabular}

Symmetry transformations used to generate equivalent atoms: 
Table S28. Anisotropic displacement parameters $\left(\AA^{2} \times 10^{3}\right)$ for $\mathbf{2 d O S O}_{2} \mathbf{C F}_{3}$. The anisotropic displacement factor exponent takes the form: $-2 \pi^{2}\left[h^{2} a^{* 2} U^{11}+\ldots+2 h k a^{*} b^{*} U^{12}\right]$

\begin{tabular}{|c|c|c|c|c|c|c|}
\hline & $\mathrm{U}^{11}$ & $\mathrm{U}^{22}$ & $\mathrm{U}^{33}$ & $U^{23}$ & $\mathrm{U}^{13}$ & $\mathrm{U}^{12}$ \\
\hline $\mathrm{P}(1)$ & $52(1)$ & $25(1)$ & $27(1)$ & $0(1)$ & $4(1)$ & $-1(1)$ \\
\hline $\mathrm{P}(2)$ & $57(1)$ & $27(1)$ & $31(1)$ & $-3(1)$ & $7(1)$ & $-6(1)$ \\
\hline $\mathrm{C}(1)$ & $99(6)$ & $48(4)$ & $37(4)$ & $-2(3)$ & $-10(4)$ & $-13(4)$ \\
\hline$C(2)$ & $81(6)$ & $71(5)$ & $76(6)$ & $-28(4)$ & $22(4)$ & $-1(4)$ \\
\hline$C(3)$ & $132(7)$ & $29(3)$ & $49(4)$ & $-4(3)$ & $16(4)$ & $-25(4)$ \\
\hline $\mathrm{C}(11)$ & $45(3)$ & $28(3)$ & $26(3)$ & $-6(2)$ & $0(2)$ & $-6(2)$ \\
\hline$C(12)$ & $51(4)$ & $32(3)$ & $41(4)$ & $4(3)$ & $4(3)$ & $-6(3)$ \\
\hline$C(13)$ & $50(4)$ & $48(4)$ & $65(5)$ & $6(3)$ & $16(3)$ & $-11(3)$ \\
\hline$C(14)$ & $37(3)$ & $42(4)$ & $61(4)$ & $-7(3)$ & $2(3)$ & $-1(3)$ \\
\hline$C(15)$ & $52(4)$ & $23(3)$ & $47(4)$ & $0(3)$ & $-4(3)$ & $-1(3)$ \\
\hline$C(16)$ & $63(4)$ & $31(3)$ & $36(3)$ & $0(3)$ & $9(3)$ & $1(3)$ \\
\hline $\mathrm{C}(21)$ & $49(3)$ & $24(3)$ & $30(3)$ & $-2(2)$ & $6(3)$ & $-2(2)$ \\
\hline$C(22)$ & $56(4)$ & $32(3)$ & $35(3)$ & $4(3)$ & $3(3)$ & $-5(3)$ \\
\hline$C(23)$ & $64(4)$ & $40(3)$ & $39(4)$ & $5(3)$ & $16(3)$ & $-7(3)$ \\
\hline$C(24)$ & $53(4)$ & $36(3)$ & $55(4)$ & $-7(3)$ & $13(3)$ & $0(3)$ \\
\hline$C(25)$ & $40(4)$ & $49(4)$ & $57(4)$ & $-3(3)$ & $1(3)$ & $0(3)$ \\
\hline$C(26)$ & $52(4)$ & $38(3)$ & $35(3)$ & $-4(3)$ & $9(3)$ & $3(3)$ \\
\hline S & $121(2)$ & $36(1)$ & $40(1)$ & $2(1)$ & $10(1)$ & $22(1)$ \\
\hline $\mathrm{F}(31)$ & $168(6)$ & $81(4)$ & $154(6)$ & $-56(4)$ & $-13(5)$ & $-31(4)$ \\
\hline $\mathrm{F}(32)$ & $145(5)$ & $162(6)$ & $65(3)$ & $21(4)$ & $58(3)$ & $38(4)$ \\
\hline $\mathrm{F}(33)$ & $114(4)$ & $123(4)$ & $69(3)$ & $14(3)$ & $8(3)$ & $59(4)$ \\
\hline $\mathrm{O}(31)$ & $390(16)$ & $95(6)$ & $115(7)$ & $-44(5)$ & $50(8)$ & $-139(8)$ \\
\hline $\mathrm{O}(32)$ & $113(5)$ & $113(5)$ & 71(4) & $28(4)$ & $51(4)$ & $49(4)$ \\
\hline $\mathrm{O}(33)$ & $236(10)$ & 197(9) & $70(4)$ & $27(5)$ & $8(5)$ & $173(8)$ \\
\hline$C(30)$ & $49(4)$ & $80(5)$ & $37(4)$ & $-3(4)$ & $3(3)$ & $7(4)$ \\
\hline
\end{tabular}


Table S29. Torsion angles $\left[^{\circ}\right]$ for $\mathbf{2} \mathbf{d O S O}_{2} \mathbf{C F}_{3}$.

\begin{tabular}{|c|c|}
\hline $\mathrm{C}(11)-\mathrm{P}(1)-\mathrm{P}(2)-\mathrm{C}(2)$ & $-157.1(4)$ \\
\hline $\mathrm{C}(21)-\mathrm{P}(1)-\mathrm{P}(2)-\mathrm{C}(2)$ & $-46.2(4)$ \\
\hline $\mathrm{C}(11)-\mathrm{P}(1)-\mathrm{P}(2)-\mathrm{C}(1)$ & $-33.1(3)$ \\
\hline $\mathrm{C}(21)-\mathrm{P}(1)-\mathrm{P}(2)-\mathrm{C}(1)$ & $77.8(4)$ \\
\hline $\mathrm{C}(11)-\mathrm{P}(1)-\mathrm{P}(2)-\mathrm{C}(3)$ & $87.0(4)$ \\
\hline $\mathrm{C}(21)-\mathrm{P}(1)-\mathrm{P}(2)-\mathrm{C}(3)$ & $-162.0(4)$ \\
\hline $\mathrm{C}(21)-\mathrm{P}(1)-\mathrm{C}(11)-\mathrm{C}(16)$ & $-33.2(6)$ \\
\hline $\mathrm{P}(2)-\mathrm{P}(1)-\mathrm{C}(11)-\mathrm{C}(16)$ & $72.6(5)$ \\
\hline $\mathrm{C}(21)-\mathrm{P}(1)-\mathrm{C}(11)-\mathrm{C}(12)$ & $144.9(4)$ \\
\hline $\mathrm{P}(2)-\mathrm{P}(1)-\mathrm{C}(11)-\mathrm{C}(12)$ & $-109.4(4)$ \\
\hline $\mathrm{C}(16)-\mathrm{C}(11)-\mathrm{C}(12)-\mathrm{C}(13)$ & $0.9(9)$ \\
\hline $\mathrm{P}(1)-\mathrm{C}(11)-\mathrm{C}(12)-\mathrm{C}(13)$ & $-177.4(5)$ \\
\hline $\mathrm{C}(11)-\mathrm{C}(12)-\mathrm{C}(13)-\mathrm{C}(14)$ & $-0.2(10)$ \\
\hline$C(12)-C(13)-C(14)-C(15)$ & $-0.7(10)$ \\
\hline$C(13)-C(14)-C(15)-C(16)$ & $0.9(9)$ \\
\hline$C(14)-C(15)-C(16)-C(11)$ & $-0.2(9)$ \\
\hline $\mathrm{C}(12)-\mathrm{C}(11)-\mathrm{C}(16)-\mathrm{C}(15)$ & $-0.7(9)$ \\
\hline $\mathrm{P}(1)-\mathrm{C}(11)-\mathrm{C}(16)-\mathrm{C}(15)$ & $177.3(4)$ \\
\hline $\mathrm{C}(11)-\mathrm{P}(1)-\mathrm{C}(21)-\mathrm{C}(26)$ & $-125.3(4)$ \\
\hline $\mathrm{P}(2)-\mathrm{P}(1)-\mathrm{C}(21)-\mathrm{C}(26)$ & $129.2(4)$ \\
\hline $\mathrm{C}(11)-\mathrm{P}(1)-\mathrm{C}(21)-\mathrm{C}(22)$ & $55.2(5)$ \\
\hline $\mathrm{P}(2)-\mathrm{P}(1)-\mathrm{C}(21)-\mathrm{C}(22)$ & $-50.2(5)$ \\
\hline $\mathrm{C}(26)-\mathrm{C}(21)-\mathrm{C}(22)-\mathrm{C}(23)$ & $-0.3(8)$ \\
\hline $\mathrm{P}(1)-\mathrm{C}(21)-\mathrm{C}(22)-\mathrm{C}(23)$ & 179.1(5) \\
\hline $\mathrm{C}(21)-\mathrm{C}(22)-\mathrm{C}(23)-\mathrm{C}(24)$ & $-1.4(9)$ \\
\hline $\mathrm{C}(22)-\mathrm{C}(23)-\mathrm{C}(24)-\mathrm{C}(25)$ & $1.6(9)$ \\
\hline $\mathrm{C}(23)-\mathrm{C}(24)-\mathrm{C}(25)-\mathrm{C}(26)$ & $-0.1(9)$ \\
\hline $\mathrm{C}(24)-\mathrm{C}(25)-\mathrm{C}(26)-\mathrm{C}(21)$ & $-1.6(9)$ \\
\hline$C(22)-C(21)-C(26)-C(25)$ & $1.8(8)$ \\
\hline $\mathrm{P}(1)-\mathrm{C}(21)-\mathrm{C}(26)-\mathrm{C}(25)$ & $-177.7(5)$ \\
\hline $\mathrm{O}(32)-\mathrm{S}-\mathrm{C}(30)-\mathrm{F}(32)$ & $-178.2(6)$ \\
\hline $\mathrm{O}(33)-\mathrm{S}-\mathrm{C}(30)-\mathrm{F}(32)$ & $-52.5(8)$ \\
\hline $\mathrm{O}(31)-\mathrm{S}-\mathrm{C}(30)-\mathrm{F}(32)$ & $65.3(7)$ \\
\hline $\mathrm{O}(32)-\mathrm{S}-\mathrm{C}(30)-\mathrm{F}(31)$ & $-60.4(7)$ \\
\hline
\end{tabular}


$\mathrm{O}(33)-\mathrm{S}-\mathrm{C}(30)-\mathrm{F}(31)$

$65.3(8)$

$\mathrm{O}(31)-\mathrm{S}-\mathrm{C}(30)-\mathrm{F}(31)$

$-177.0(7)$

$\mathrm{O}(32)-\mathrm{S}-\mathrm{C}(30)-\mathrm{F}(33)$

$53.8(7)$

$\mathrm{O}(33)-\mathrm{S}-\mathrm{C}(30)-\mathrm{F}(33)$

$179.5(7)$

$\mathrm{O}(31)-\mathrm{S}-\mathrm{C}(30)-\mathrm{F}(33)$

$-62.8(7)$

Symmetry transformations used to generate equivalent atoms: 